NISTIR 85-3273-3

(Rev. 11/88)

\title{
Energy Prices and Discount Factors for Life-Cycle Cost Analysis 1988
}

Annual Supplement to

NBS Handbook 135 and

NBS Special Publication 709
Barbara C Lippiatt Rosalie T. Ruegg

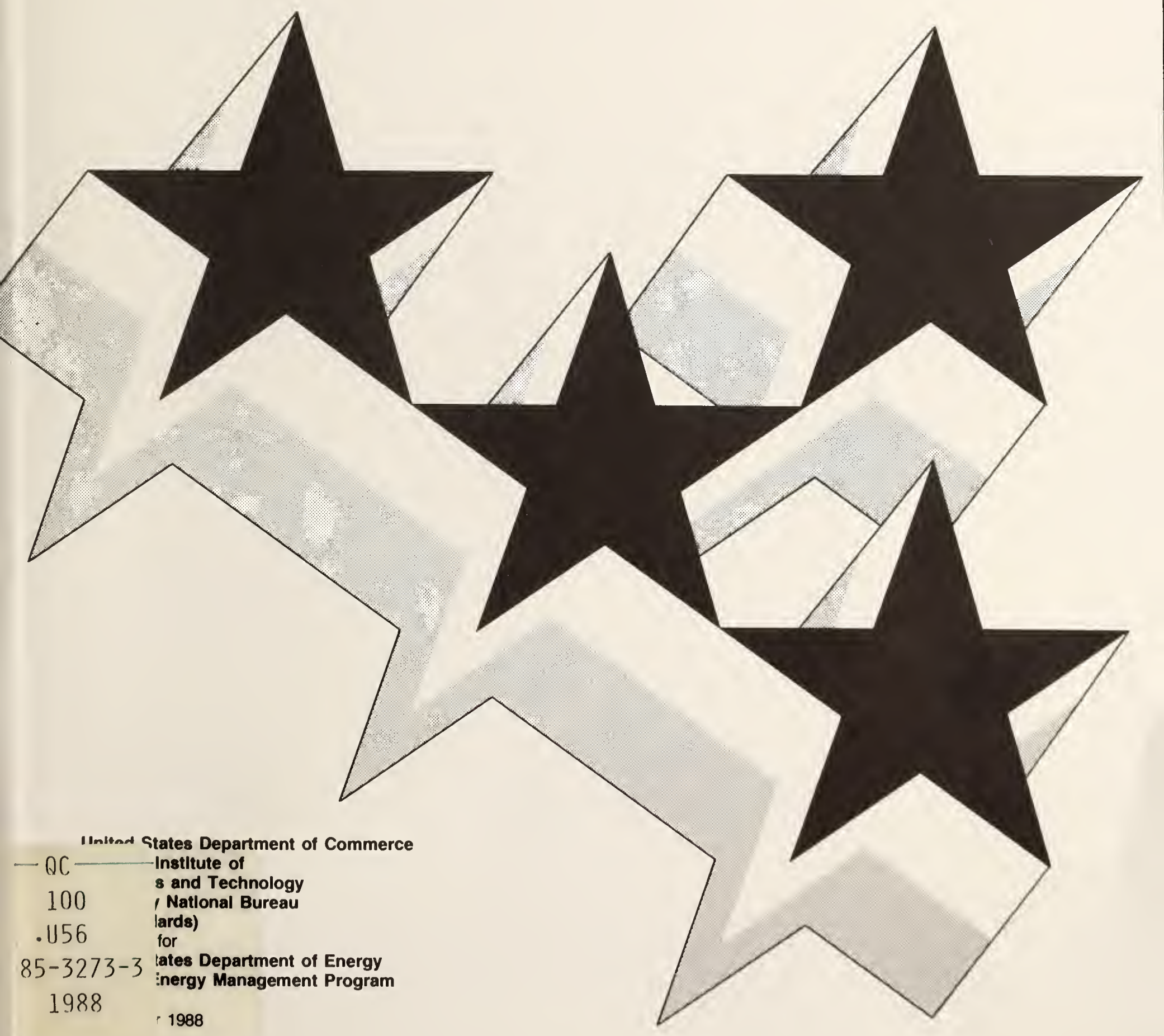


NISTIR 85-3273-3

(Rev. 11/88)

\section{ENERGY PRICES AND DISCOUNT FACTORS FOR LIFE-CYCLE COST ANALYSIS 1988}

Annual Supplement to

NBS Handbook 135 and

NBS Special Publication 709

(Data for the Federal Methodology for Life-Cycle Cost Analysis, Title10, CFR, Part 436, Subpart A)

Barbara C. Lippiatt

Rosalie T. Ruegg

\section{U.S. DEPARTMENT OF COMMERCE}

National Institute of

Standards and Technology

(Formerly National Bureau

of Standards)

Applied Economics Group

Center for Computing and

Applied Mathematics

Gaithersburg, MD 20899

November 1988

Prepared for:

U.S. Department of Energy

Office of the Assistant Secretary for

Conservation and Renewable Energy

Federal Programs Office

Washington, DC 20585

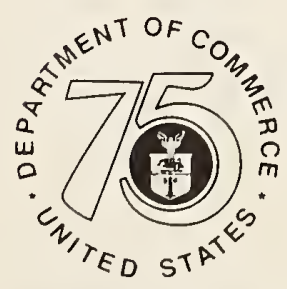

NOTE: As of 23 August 1988, the National Bureau of Standards (NBS) became the National Institute of Standards and Technology (NIST) when President Reagan signed into law the Omnibus Trade and 



\section{PREFACE}

This is the 1988 edition of energy prices and discount factors for life-cycle cost analysis as established by the U.S. Department of Energy (DoE) in Subpart A of Part 436 of Title 10 of the Code of Federal Regulations (10 CFR Part 436, Subpart A), which is entitled "Federal Energy Management and Planning Program" (FEMP). The data are provided as an aid to implementing life-cycle cost evaluations of potential energy conservation and renewable energy investments in existing and new federally owned and leased buildings. Life-cycle cost evaluations of energy conservation and renewable energy investments in Federal buildings are required by Section 381 (a)(2) of the Energy Policy and Conservation Act (EPCA), as amended, 42 U.S.C. 6361(a)(2); by Section 10 of Executive Order 11912, as amended by Executive Order 120003; and by Title $V$ of the National Energy Conservation Policy Act (NECPA), 92 Stat. 3275, as amended by Section 405 of the Energy Security Act, 94 Stat. 611.

As called for by legislation, the National Institute of Standards and Technology (formerly National Bureau of Standards) has provided technical assistance to the U.S. Department of Energy in formulating the life-cycle costing methods and procedures for implementation. It has developed this report as the second of a three-volume set which together provide the methods, data, and computational tools for life-cycle cost analysis of Federal energy projects.

Included in the three-volume set for Federal life-cycle cost analysis are the following:

(1) Life-Cycle Costing Manual for the Federal Energy Management Program, National Bureau of Standards, Handbook 135 (revised 1987).

The manual is a guide to understanding life-cycle costing and related methods of economic analysis as they are applied to Federal decisions. It describes the required procedures and assumptions, defines and explains how to apply and interpret economic performance measures, gives examples of Federal decision problems and their solutions, and provides worksheets and other computational aids and instructions for calculating the required measures.

(2) Energy Prices and Discount Factors for Life-Cycle Cost Analysis,

National Institute of Standards and Technology, NISTIR 85-3273

(updated annually).

This report, which is updated annually, gives the energy price and discount factor multipliers needed to estimate the present value of energy and other future costs. The data are based on energy price projections developed by the Energy Information Administration of the U.S. Department of Energy. Request the latest edition when ordering. 
(3) A User's Guide to the Federal Building Life-Cycle Cost (FBLCC) Computer Program, National Bureau of Standards, NBS TN 1222 (Computer program revised periodically).

This report is a user's guide to the computer program, "FBLCC." FBLCC, designed to run on an IBM PC/XT/AT, or compatible microcomputer, can be used to calculate the life-cycle costs, net savings, and savings-to-investment ratios of Federal energy projects, consistent with the procedures and assumptions described in Handbook 135 (see \#1 above) and incorporating the energy price data of the most recent issue of NISTIR 85-3273 (see \#2 above). FBLCC generates reports which summarize the assumptions and results in tabular form. Information for ordering the FBLCC computer program disk is provided in the User's Guide.

To order any of these publications, contact:

$$
\begin{aligned}
& \text { Advanced Sciences, Inc. } \\
& 2000 \text { North 15th Street } \\
& \text { Suite } 407 \\
& \text { Arlington, VA } 22201 \\
& \text { Telephone (703) 243-4900 }
\end{aligned}
$$

Please request the publication by name and number.

The life-cycle costing methods and procedures set forth in 10 C.F.R., Part 436, Subpart A, are to be followed by all Federal agencies, unless specifically exempted, in evaluating the cost effectiveness of potential energy conservation and renewable energy investments in federally owned and leased buildings.

Though aimed specifically at supporting the economic evaluation of Federal building projects which are classified as energy conservation or renewable energy projects, the three-volume set can also be used to perform economic evaluations of Federal building projects which are not primarily for conserving energy or providing renewable energy but which have an energy cost component. Both applications are explained in the three reports.

*FBLCC Version SE2.1 incorporates the 1988 energy price data contained in NISTIR 85-3273-3. 
The U.S. Department of Energy was also directed by legislation and executive order to make available to the private sector the methods, procedures, and related aids developed for Federal use. In response to this directive, the National Institute of Standards and Technology, under sponsorship by the U.S. Department of Energy, has published an additional life-cycle costing book for use by the private sector entitled Comprehensive Guide for Least-Cost Energy Decisions, NBS SP 709 (January 1987). The private sector book is also supported by the data provided here, as well as by a special version of the computer program adapted to private sector analysis ("NBSLCC"). This report may be ordered from Advanced Sciences, Inc. at the above address. Information for ordering the NBSLCC computer disk is provided in NBS SP 709.

Further information on the Federal Energy Management Program can be obtained from the Federal Energy Management Program Staff, Office of the Assistant Secretary for Conservation and Renewable Energy, U.S. Department of Energy. Please direct communication to: FEMP, CE 10.1, U.S. Department of Energy, 1000 Independence Avenue, SW, Washington, DC 20585. 
This is the 1988 annual edition of energy prices and discount factors for performing life-cycle cost analyses of energy conservation and renewable energy projects. It supports the Federal life-cycle costing methodology as described in NBS Handbook 135 (HB 135) and private sector life-cycle cost analysis as described in NBS Special Publication 709 (SP 709). Tables A, B, and $C$ are revisions of appendices $A, B$, and $C$, respectively, of $H B 135$. Tables $\mathrm{A}(7 \%), \mathrm{Ba}$, and $\mathrm{C}$ apply to Federal energy conservation and renewable energy projects. Tables $A(108), B b$, and $C$ apply to Federal projects that require energy price forecasts but are not primarily energy conserving. Tables $S$, in the last section of this report, are revisions to appendix $B$, Part I of SP 709 and are provided for the convenience of private sector analysts wishing to make use of Federal energy price forecasts. 
The authors wish to thank Mr. Richard W. Brancato, Head of the Federal Programs Office, and Mr. Dean DeVine, also of the Federal Programs Office, for their continued support and direction of this work. Appreciation is also extended to Dr. Richard D. Farmer, Chief of the Supply Analysis and Integration Branch of the Energy Information Administration, and Dr. Eugene Reiser, also of the Supply Analysis and Integration Branch, for providing energy price projections and escalation rates. Dr. Stephen Weber of the Applied Economics Group of the National Institute of Standards and Technology deserves special thanks for guidance in developing equations and computer programming. Credit is due Ms. Sieglinde Fuller, Ms. Laurene Linsenmayer, and Ms. Katherine Johnson, also of the Applied Economics Group, for assistance in computer programming and manuscript preparation. 
PREFACE . . . . . . . . . . . . . . . . . . . . . . . . . . 111

ABSTRACT. . . . . . . . . . . . . . . . . . . . . . . . . vi

ACKNOWLEDGMENTS . . . . . . . . . . . . . . . . . . . . . . . vil

CONTENTS. . . . . . . . . . . . . . . . . . . . . . . . . . . vili

LIST OF TABLES . . . . . . . . . . . . . . . . . . . . . . . . . IX

SINGLE PRESENT WORTH AND UNIFORM PRESENT WORTH DISCOUNT FACTORS . . . . 1

MODIFIED UNIFORM PRESENT WORTH DISCOUNT FACTORS FOR FEDERAL USE . . . . 5

1988 AVERAGE FUEL PRICES, PROJECTED AVERAGE FUEL PRICE INDICES, AND

PROJECTED AVERAGE FUEL PRICE ESCALATION RATES FOR FEDERAL USE . . . . . 18

PROJECTED AVERAGE FUEL PRICE INDICES FOR PRIVATE SECTOR USE . . . . . . 37 


\section{LIST OF TABLES}

Page

A-1. SPW Factors for Finding the Present Value of Future Nonfuel, Nonannually Recurring Amounts . . . . . . . . . . . . . . 3

A-2. UPW Factors for Finding the Present Value of Future Nonfuel, Annually Recurring Amounts. . . . . . . . . . . . . . . . . 4

B-1a. UPW ${ }^{*}$ Discount Factors Adjusted for Average Fuel Price Escalation, by End-Use Sector and Major Fuel. Discount Rate $=7$ Percent. Census Region 1 (Maine, New Hampshire, Vermont, Massachusetts, Connecticut, Rhode Island, New York, New Jersey, Pennsylvania) . . . . . . . . . . . . . . . .

B-2a. $\quad$ UPW $^{*}$ Discount Factors Adjusted for Average Fuel Price Escalation, by End-Use Sector and Major Fuel. Discount Rate $=7$ Percent. Census Region 2 (Ohio, Indiana, Illinois, Michigan, Wisconsin, Minnesota, Iowa, Missouri, North Dakota, South Dakota, Nebraska, Kansas) . . . . . . . . . . . . . .

B-3a. UPW Discount Factors Adjusted for Average Fuel Price Escalation, by End-Use Sector and Major Fuel. Discount Rate $=7$ Percent. Census Region 3 (Delaware, Maryland, District of Columbia, Virginia, West Virginia, North Carolina, South Carolina, Georgia, Florida, Kentucky, Tennessee, Alabama, Mississippi, Arkansas, Louisiana, Oklahoma, Texas)

B-4a. UPW* Discount Factors Adjusted for Average Fuel Price Escalation, by End-Use Sector and Major Fuel. Discount Rate $=7$ Percent. Census Region 4 (Montana, Idaho, Wyoming, Colorado, New Mexico, Arizona, Utah, Nevada, Washington, Oregon, California, Alaska, Hawaii)

B-5a. $\quad$ UPW $^{\star}$ Discount Factors Adjusted for Average Fuel Price Escalation, by End-Use Sector and Major Fuel. Discount Rate $=7$ Percent. United States Average. . . . . . . . . .

B-1b. UPW Discount Factors Adjusted for Average Fuel Price Escalation, by End-Use Sector and Major Fuel. Discount Rate $=10$ Percent. Census Region 1 (Maine, New Hampshire, Vermont, Massachusetts, Connecticut, Rhode Island, New York, New Jersey, Pennsylvania)

B-2b. $\quad$ UPW $^{\star}$ Discount Factors Adjusted for Average Fuel Price Escalation, by End-Use Sector and Major Fuel. Discount Rate $=10$ Percent. Census Region 2 (Ohio, Indiana, Illinois, Michigan, Wisconsin, Minnesota, Iowa, Missouri, North Dakota, South Dakota, Nebraska, Kansas) . . . . . . . . 14 
B-3b. UPW* Discount Factors Adjusted for Average Fuel Price Escalation, by End-Use Sector and Major Fuel. Discount Rate $=10$ Percent. Census Region 3 (Delaware, Maryland, District of Columbia, Virginia, West Virginia, North Carolina, South Carolina, Georgia, Florida, Kentucky, Tennessee, Alabama, Mississippi, Arkansas, Louisiana, Oklahoma, Texas).

B-4b. UPW Discount Factors Adjusted for Average Fuel Price Escalation, by End-Use Sector and Major Fuel. Discount Rate $=10$ Percent. Census Region 4 (Montana, Idaho, Wyoming, Colorado, New Mexico, Arizona, Utah, Nevada, Washington, Oregon, California, Alaska, Hawaii) . . . . . 16

B-5b. UPW Discount Factors Adjusted for Average Fuel Price Escalation, by End-Use Sector and Major Fuel. Discount Rate $=10$ Percent. United States Average . . . . . . . . .

C. Regional and U.S. Average 1988 Fuel Prices, by End-Use Sector and Major Fuel . . . . . . . . . . . . . . . 20

Ca-1. Projected Average Fuel Price Indices Tied to the Value of the Dollar in 1988, by End-Use Sector and Major Fuel. Census Region 1 (Maine, New Hampshire, Vermont, Massachusetts, Connecticut, Rhode Island, New York, New Jersey, Pennsylvania) . . . . . . . . . . . . . . 22

Ca-2. Projected Average Fuel Price Indices Tied to the Value of the Dollar in 1988, by End-Use Sector and Major Fuel. Census Region 2 (Ohio, Indiana, Illinois, Michigan, Wisconsin, Minnesota, Iowa, Missouri, North Dakota, South Dakota, Nebraska, Kansas) . . . . . . . . . . . 24

Ca-3. Projected Average Fuel Price Indices Tied to the Value of the Dollar in 1988, by End-Use Sector and Major Fuel.

Census Region 3 (Delaware, Maryland, District of Columbia, Virginia, West Virginia, North Carolina, South Carolina, Georgia, Florida, Kentucky, Tennessee, Alabama, Mississippi, Arkansas, Louisiana, Oklahoma, Texas).

Ca-4. Projected Average Fuel Price Indices Tied to the Value of the Dollar in 1988, by End-Use Sector and Major Fuel. Census Region 4 (Montana, Idaho, Wyoming, Colorado, New Mexico, Arizona, Utah, Nevada, Washington, Oregon, California, Alaska, Hawaii) 
Ca-5. Projected Average Fuel Price Indices Tied to the Value of the Dollar in 1988, by End-Use Sector and Major Fuel.

United States Average . . . . . . . . . . . . . . . . . . .

$\mathrm{Cb}-1$. Projected Average Fuel Price Escalation Rates Exclusive of General Price Inflation, by End-Use Sector and Major Fuel (Percentage Change Compounded Annually). Census Region 1 (Maine, New Hampshire, Vermont, Massachusetts, Connecticut, Rhode Island, New York, New Jersey, Pennsylvania) . . . . . .

$\mathrm{Cb}-2$. Projected Average Fuel Price Escalation Rates Exclusive of General Price Inflation, by End-Use Sector and Major Fuel (Percentage Change Compounded Annually). Census Region 2 (Ohio, Indiana, Illinois, Michigan, Wisconsin, Minnesota, Iowa, Missouri, North Dakota, South Dakota, Nebraska,

Kansas).

$\mathrm{Cb}-3$. Projected Average Fuel Price Escalation Rates Exclusive of General Price Inflation, by End-Use Sector and Major Fuel (Percentage Change Compounded Annually). Census Region 3 (Delaware, Maryland, District of Columbia, Virginia, West Virginia, North Carolina, South Carolina, Georgia, Florida, Kentucky, Tennessee, Alabama, Mississippi, Arkansas, Louisiana, Oklahoma, Texas). . . . . . . . . . . . . 34

$\mathrm{Cb}-4$. Projected Average Fuel Price Escalation Rates Exclusive of General Price Inflation, by End-Use Sector and Major Fuel (Percentage Change Compounded Annually). Census Region 4 (Montana, Idaho, Wyoming, Colorado, New Mexico, Arizona, Utah, Nevada, Washington, Oregon, California, Alaska, Hawaii)

$\mathrm{Cb}-5$. Projected Average Fuel Price Escalation Rates Exclusive of General Price Inflation, by End-Use Sector and Major Fuel (Percentage Change Compounded Annually). United States Average

S-1. Projected Average Fuel Price Indices with Assumed General Price Inflation Rates of $0,5,10$, and 15 Percent, by EndUse Sector and Major Fuel. Census Region 1 (Maine, New Hampshire, Vermont, Massachusetts, Connecticut, Rhode Island, New York, New Jersey, Pennsylvania).

S-2. Projected Average Fuel Price Indices with Assumed General Price Inflation Rates of 0, 5, 10, and 15 Percent, by EndUse Sector and Major Fuel. Census Region 2 (Ohio, Indiana, Illinois, Michigan, Wisconsin, Minnesota, Iowa, Missouri, North Dakota, South Dakota, Nebraska, Kansas) . . . . . . . 42 
S-3. Projected Average Fuel Price Indices with Assumed General Price Inflation Rates of 0,5, 10, and 15 Percent, by EndUse Sector and Major Fuel. Census Region 3 (Delaware, Maryland, District of Columbia, Virginia, West Virginia, North Carolina, South Carolina, Georgia, Florida, Kentucky, Tennessee, Alabama, Mississippi, Arkansas, Louisiana, Oklahoma, Texas).

S-4. Projected Average Fuel Price Indices with Assumed General Price Inflation Rates of 0, 5, 10, and 15 Percent, by EndUse Sector and Major Fuel. Census Region 4 (Montana, Idaho, Wyoming, Colorado, New Mexico, Arizona, Utah, Nevada, Washington, Oregon, California, Alaska, Hawaii) . . . . . . . 46

S-5. Projected Average Fuel Price Indices with Assumed General Price Inflation Rates of $0,5,10$, and 15 Percent, by EndUse Sector and Major Fuel. United States Average . . . . . 48 
Table A-1 of this section presents the single present worth (SPW) factors for finding the present value of future nonfuel, nonannually recurring amounts, such as repair and replacement costs and salvage values. The formula for finding the present value $(P)$ of a future amount ( $F$ ) is the following:

$$
P=F \times \underset{(1+d)^{N}}{-1}=F \times S P W_{N} \text { Factor }
$$

where $\mathrm{d}=$ Discount rate; and

$\mathrm{N}=$ Number of discount or compound interest periods; in this case the number of years until $F$ occurs.

Table A-2 presents uniform present worth (UPW) factors for finding the present value of future nonfuel amounts recurring annually, such as routine maintenance costs. The formula for finding the present value $(P)$ of an annually recurring uniform amount (A) is the following:

$$
P=A \times \frac{(1+d)^{N}-1}{d(1+d)^{N}}=A \times U_{N} \text { Factor, }
$$

where $N=$ the number of discount or compound interest periods; in this case the number of years over which A recurs.

In each table the factors are given for both 7 percent and 10 percent discount rates. The factors based on 7 percent are for finding the present value of future amounts associated with Federal energy conservation and renewable energy projects. The factors based on 10 percent are for finding the present value of future amounts associated with most other Federal projects (except those specifically exempted from the 10 percent rate). Both the 7 and 10 percent rates are defined for Federal use to be "real" rates exclusive of general price inflation. The factors are applied as multipliers to future amounts which are stated in "constant" dollars, that is, exclusive of general price inflation.

\section{Examples of How to Use the Factors:}

SPW $(7 \%)$ : To compute the present value of a replacement cost in the 8 th year for an energy efficient heating system, go to table A-1, find the 7 percent SPW factor for year $8(0.58)$, and multiply by the replacement cost.

SPW(10\%): To compute the present value of a repair cost in the 5 th year for a floor, go to table $A-1$, find the 10 percent SPW factor for year $5(0.62)$, and multiply by the repair cost.

UPW (78): To compute the present value of an annually recurring maintenance cost for a renewable energy system over 20 years, go to table A-2, find the 7 percent UPW factor for 20 years (10.59), and multiply by the annual maintenance cost. 
UPW(108): The compute the present value of annually recurring costs of office cleaning over 30 years, go to table A-2, find the 10 percent UPW factor for 30 years (9.43), and multiply by the annual cleaning cost.

For further explanation of the use of these factors, see NBS Handbook 135 , section 2.5 . 
Table A-1. SPW Factors for Finding the Present Value of Future Nonfuel, Nonannually Recurring Amounts

Number of Years

Until Future Amount Occurs (N)

$\begin{array}{ll}\text { SPW Factor } & \text { SPW Factor } \\ (d=0.07) & (d=0.10)\end{array}$

\begin{tabular}{|c|c|c|}
\hline 1 & 0.93 & 0.91 \\
\hline 2 & 0.87 & 0.83 \\
\hline 3 & 0.82 & 0.75 \\
\hline 4 & 0.76 & 0.68 \\
\hline 5 & 0.71 & 0.62 \\
\hline 6 & 0.67 & 0.56 \\
\hline 7 & 0.62 & 0.51 \\
\hline 8 & 0.58 & 0.47 \\
\hline 9 & 0.54 & 0.42 \\
\hline 10 & 0.51 & 0.39 \\
\hline 11 & 0.48 & 0.35 \\
\hline 12 & 0.44 & 0.32 \\
\hline 13 & 0.41 & 0.29 \\
\hline 14 & 0.39 & 0.26 \\
\hline 15 & 0.36 & 0.24 \\
\hline 16 & 0.34 & 0.22 \\
\hline 17 & 0.32 & 0.20 \\
\hline 18 & 0.30 & 0.18 \\
\hline 19 & 0.28 & 0.16 \\
\hline 20 & 0.26 & 0.15 \\
\hline 21 & 0.24 & 0.14 \\
\hline 22 & 0.23 & 0.12 \\
\hline 23 & 0.21 & 0.11 \\
\hline 24 & 0.20 & 0.10 \\
\hline 25 & 0.18 & 0.09 \\
\hline
\end{tabular}

$* * *$ Extended Series $* * *$

26

27

28

29

30
0.08

0.08

0.07

0.06

0.06 
Table A-2. UPW Factors for Finding the Present Value of Future Nonfuel, Annually Recurring Amounts

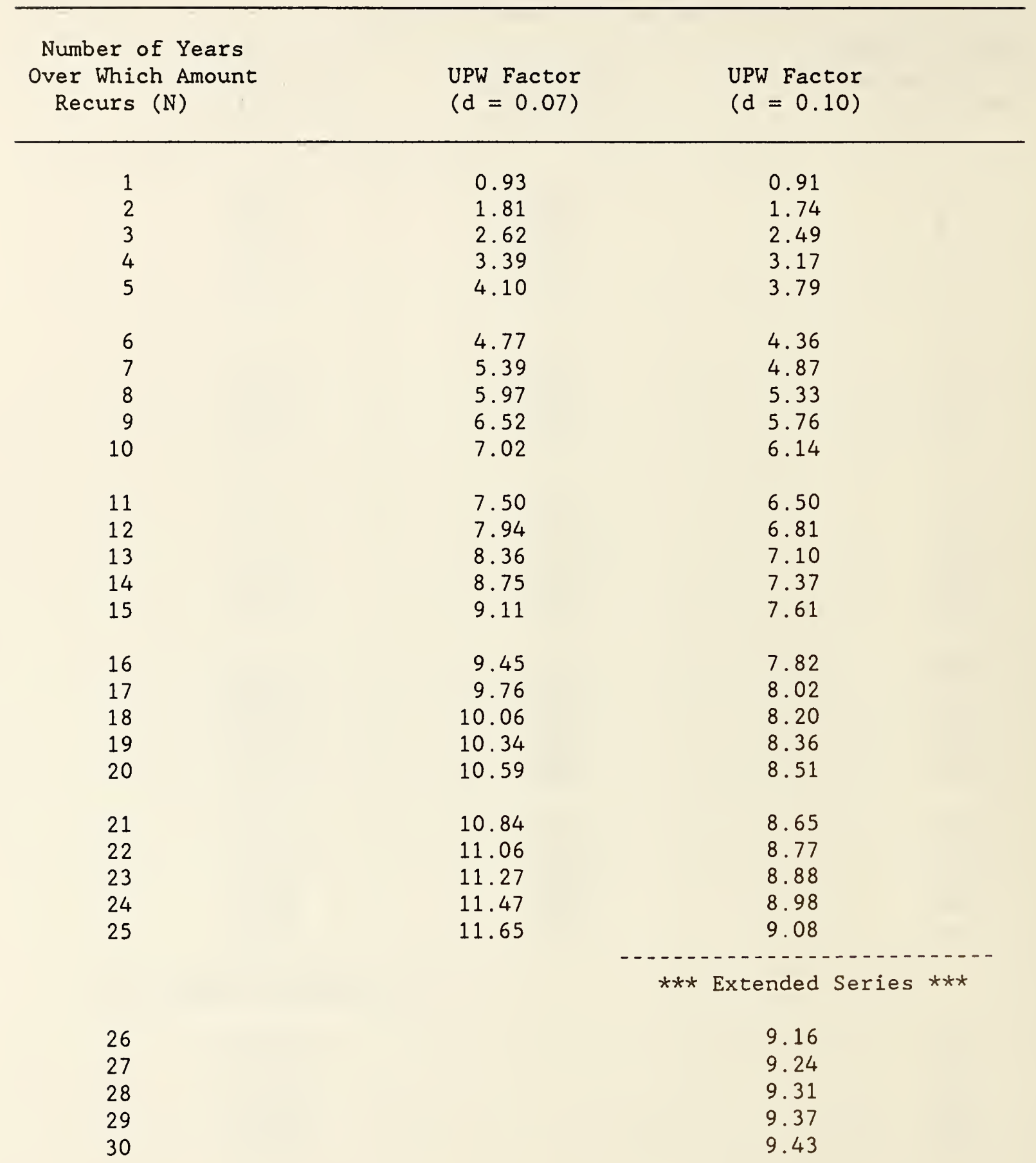


MODIFIED UNIFORM PRESENT WORTH DISCOUNT FACTORS FOR FEDERAL USE (BASED ON FEDERALLY REQUIRED DISCOUNT RATES OF SEVEN AND TEN PERCENT AND DOE-PROJECTED RATES OF CHANGE IN ENERGY PRICES, BOTH OF WHICH EXCLUDE GENERAL PRICE INFLATION)

This section presents "modified" uniform present worth (UPW") discount factors for the 4 Census regions and for the United States. The factors are modified in the sense that they incorporate projected energy price changes. There are two sets of tables: the "a" tables present UPW" factors based on a seven percent "real" discount rate (i.e., a discount rate exclusive of the rate of general price inflation), and the "b" tables present UPW" factors based on a ten percent real discount rate. The factors presented in the "a" tables are for energy costs or savings accruing over 1 to 25 years and are to be used in life-cycle cost analyses of Federal energy conservation and renewable energy projects. The factors presented in the "b" tables are for energy costs or savings accruing over 1 to 30 years and are to be used for life-cycle cost analysis of the energy component of Federal projects that are not primarily for conserving energy or providing renewable energy. The energy price projections underlying the $U W^{*}$ factors are stated as annual averages. Therefore, the factors are not tied to a particular calendar date in the year. ${ }^{1}$

For the period 1988-2018, the UPW factors incorporate rates of change in energy prices computed from indices projected by the Energy Information Administration (EIA) of the U.S. Department of Energy. Documentation on the PC-AEO model used by EIA to project energy prices is available in PC-AEO Forecasting Model for the Annual Energy Outlook 1987 (DOE/EIA-M029), June 1988. Assumptions underlying the PC-AEO model are presented by EIA in Assumptions for the Annual Energy Outlook 1987 (SR/EAFD/88-02), October 1988. Projections at the national level are reported by EIA in the Annual Energy Outlook 1987 (AEO) (DOE/EIA-0383(87), March 1988. Regional projections are reported by EIA in Regional Projections of End-Use Energy Consumption and Prices through 2000 (SR/EAFD/88-01), October 1988, and Average Regional Energy Price Projections to the Year 2013 (SR/EAFD/88-03), October 1988.

The formula for finding the present value (P) of future energy costs or savings is the following:

$$
P=A_{0} \times \sum_{n=1}^{N} \frac{I(1988+n)}{(1+d)^{n}}=A_{0} \times U^{N} W_{N}^{*} \text { Factor },
$$

where $A_{0}$ = Base-year dollar cost of energy, i.e., the annual quantity of energy times its 1988 price;

$\mathrm{n}$ = Counter used to designate each year, with $\mathrm{n}=1$ for the year 1989;

${ }^{1}$ Prior to the 1987 edition of this report, however, cash flows were assumed to begin in July since projections were stated as mid-year values. 
$N$ = Number of discount or compound interest periods; in this case the number of years over which energy costs or savings accrue;

$I_{(1888+n)}=$ Projected average fuel price index given in tables Ca-1 through $\mathrm{Ca}-5$ for the year $1988+n$; and

$\mathrm{d}=$ Discount rate.

Examples of How to Use UPW* Factors:

$\mathrm{UPW}^{*}(78)$ : To compute the present value of heating with distillate oil for an energy-conserving design of a Federal office building in New Mexico for 15 years, go to table B-4a, find the UPW factor for commercial distillate for 15 years (12.31), and multiply by the annual heating cost in 1988 dollars.

$\mathrm{UPW}^{*}(108)$ : To compute the present value of electricity costs over 30 years associated with a renovated Federal office building in Ohio, go to table B-2b, find the $U U^{*}$ factor for commercial electricity for 30 years (9.17), and multiply by the annual electricity cost in 1988 dollars.

For further explanation of the use of UPW* factors, see NBS Handbook 135 , section 2.5 .

Note: The data in the tables which follow are now reported by 4 Census regions. Prior to the 1988 edition, regional data were presented by 10 DoE regions. Figure B-1 presents a map showing the states corresponding to the 4 Census regions. The Census regions do not include American Samoa, Canal Zone, Guam, Puerto Rico, Trust Territory of the Pacific Islands, or the Virgin Islands. Analysts of Federal projects in these areas should use data which are "reasonable under the circumstances," and may refer to the U.S. average data for guidance. 


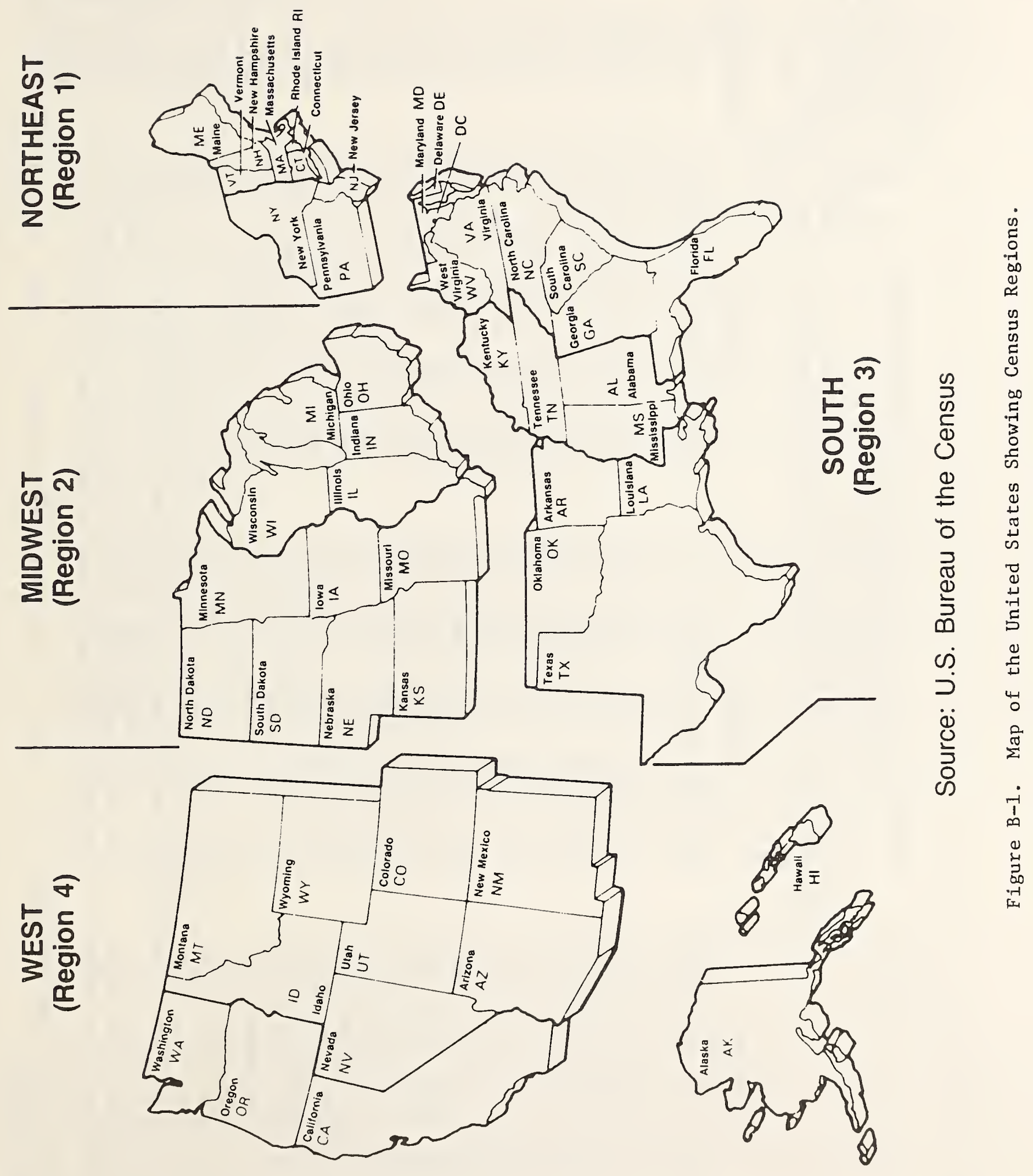



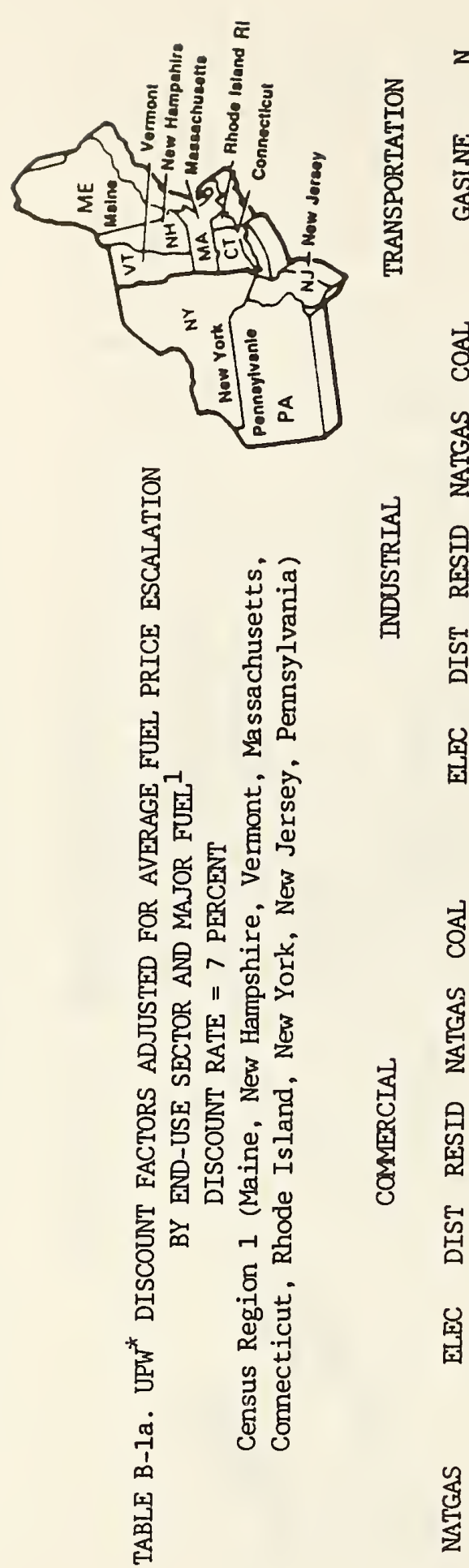

荇号

急
엉

总

星

点

量

步

星

它

国
ชุํำ

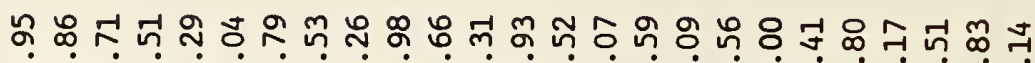

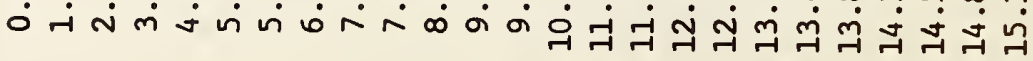

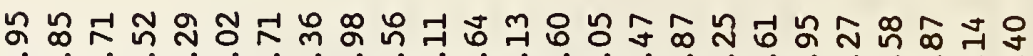
- न N

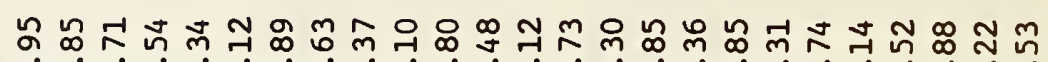
○ -

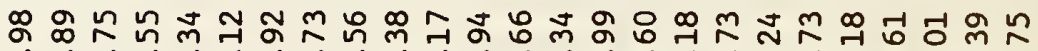

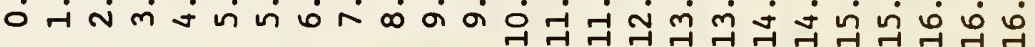

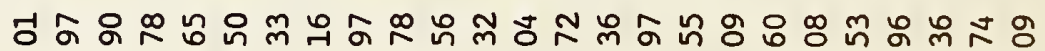

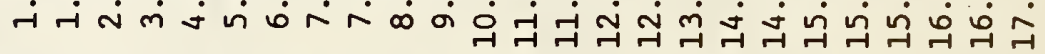

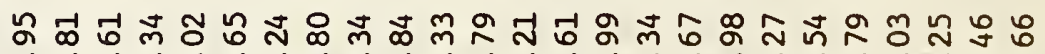

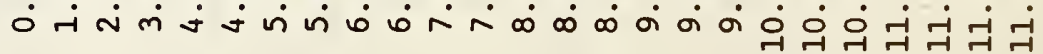

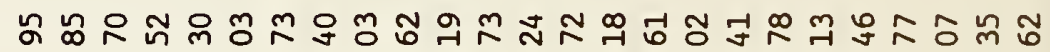
○

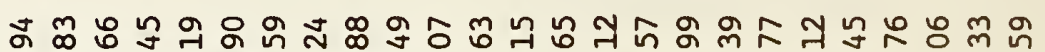
○ न

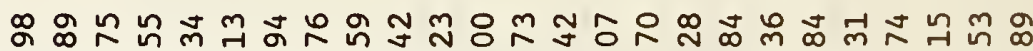

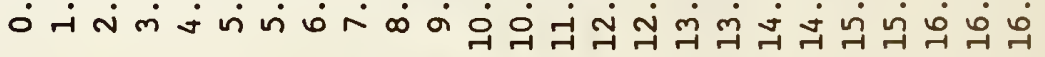

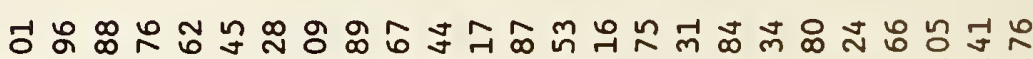
-

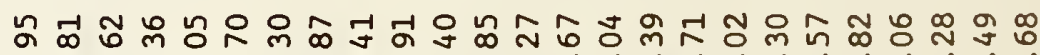

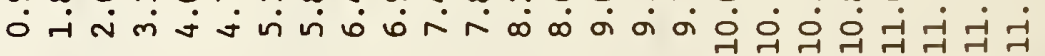

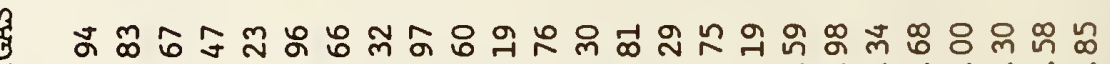

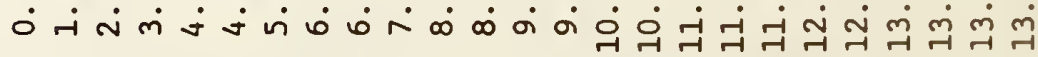

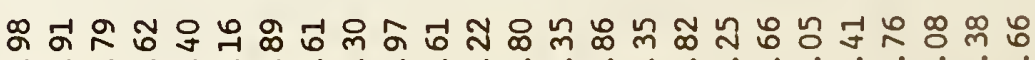

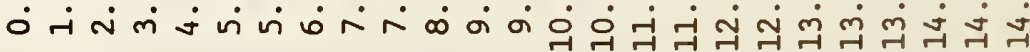

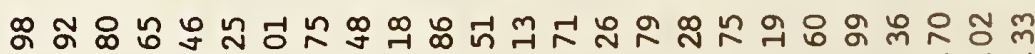

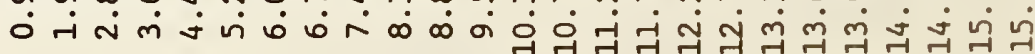
品 - 


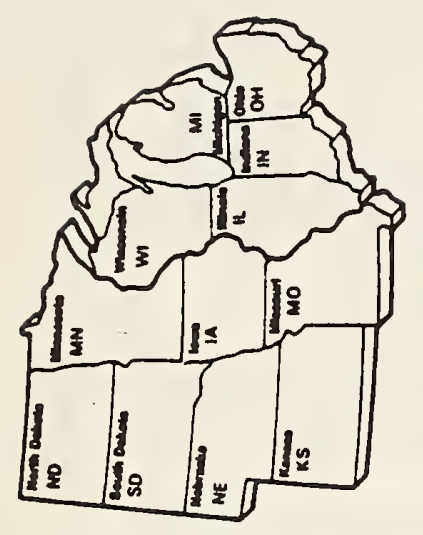

尔

Z

- $N$ m

惯

ผ 0

多

๙

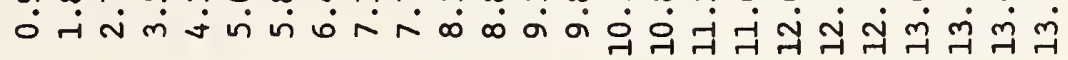

岕

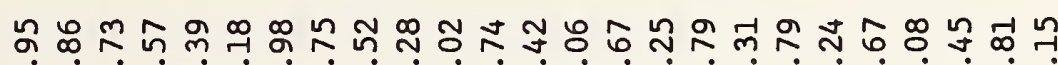

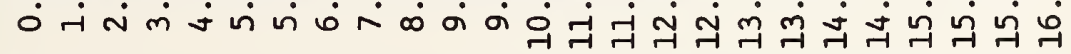

ğ

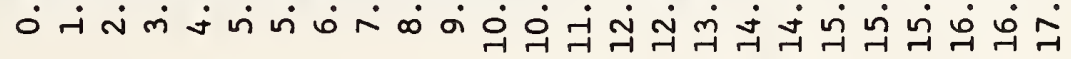

旨

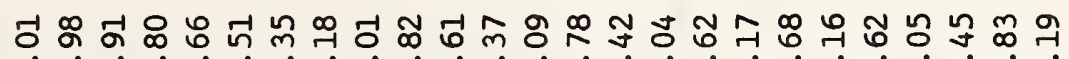

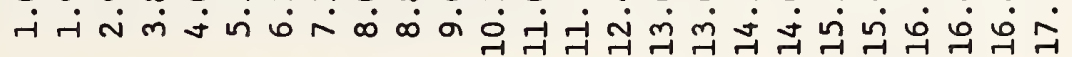

多 ๙ 0 -

농

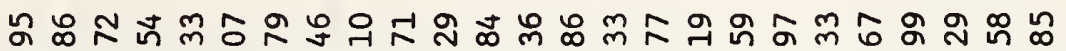

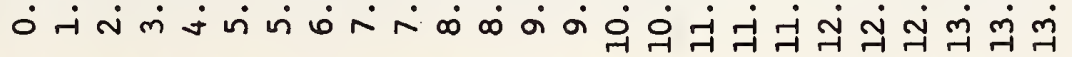

悬人 i 要

॥

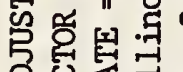

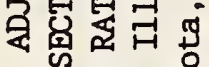

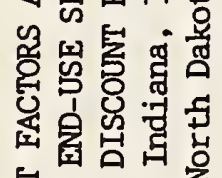

点品

近

过

๙

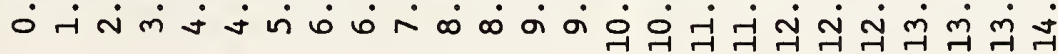

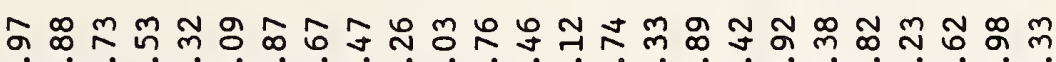

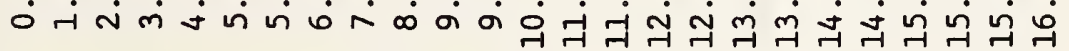

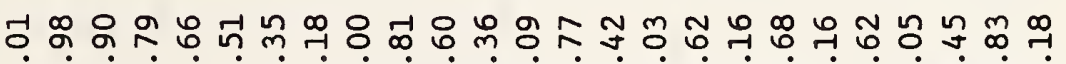

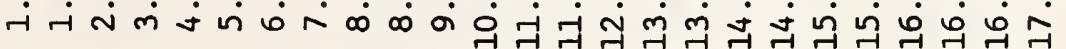
ตั 옷

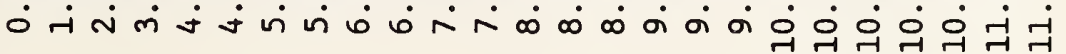

发

นิ์ -

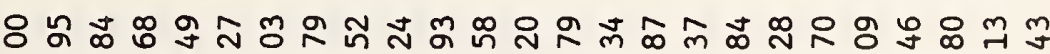

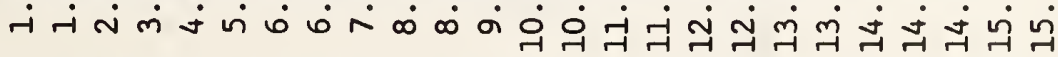

ต

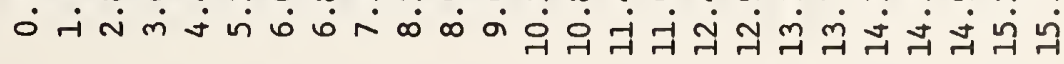

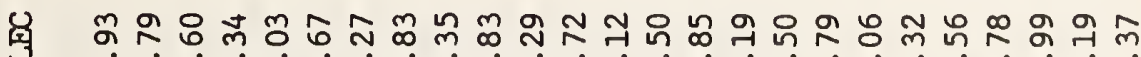

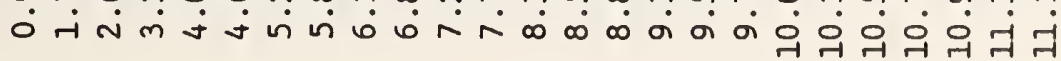



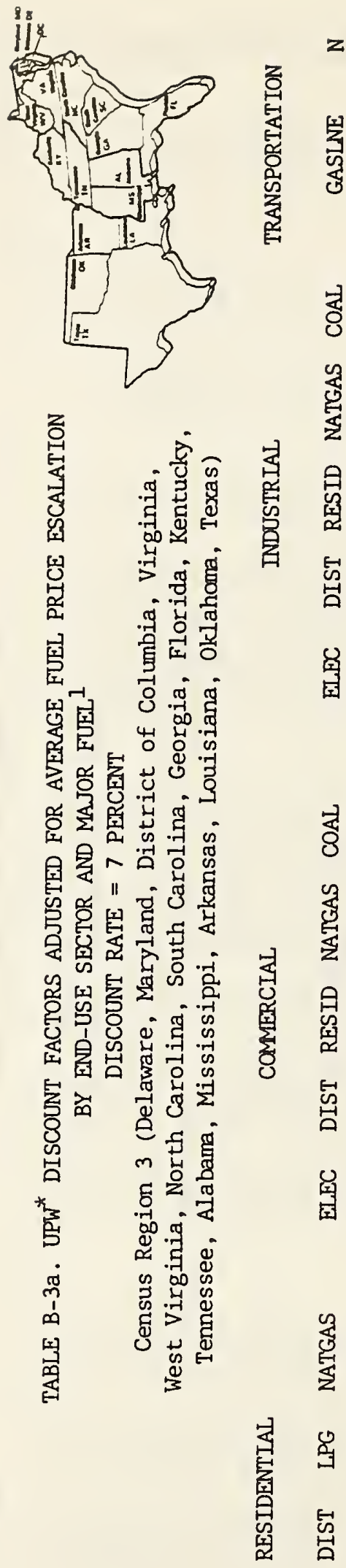

z

엉

起

봉

点

国

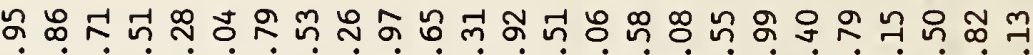

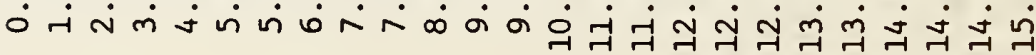

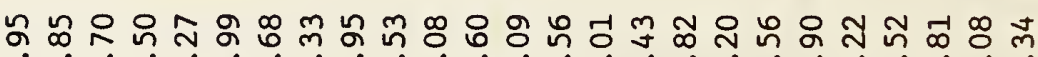

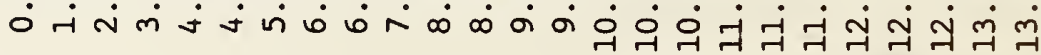

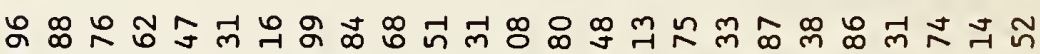
0 -

品 옷 ᅫำ

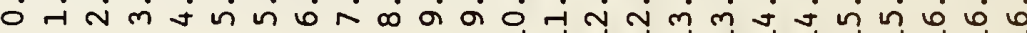

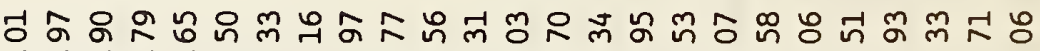
-

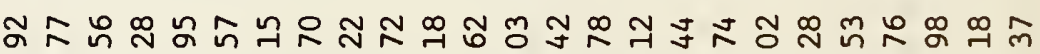

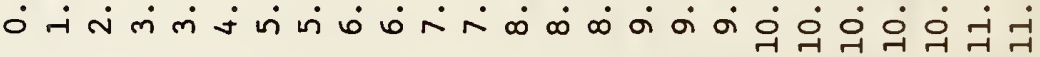

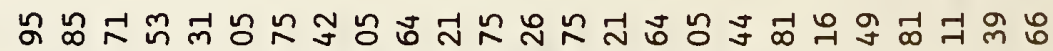

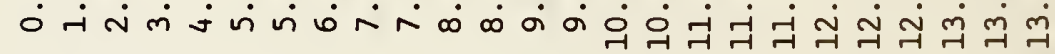

๙

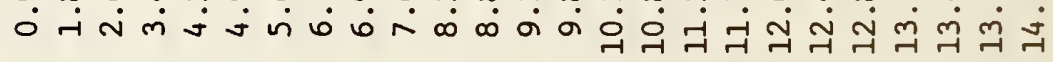

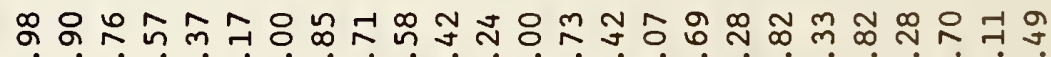

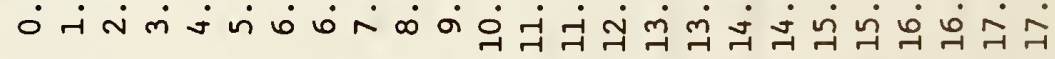

бำ -

ตำ

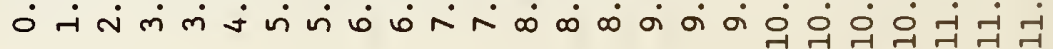

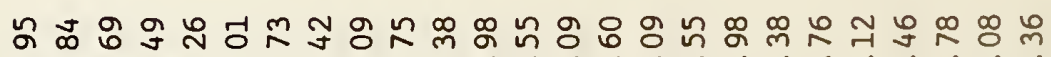

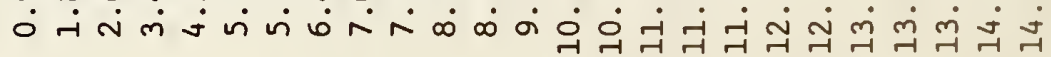

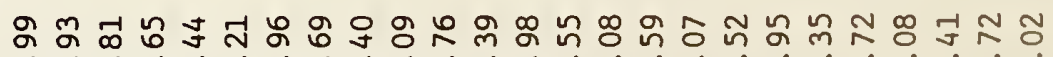

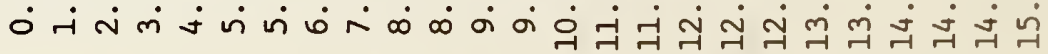

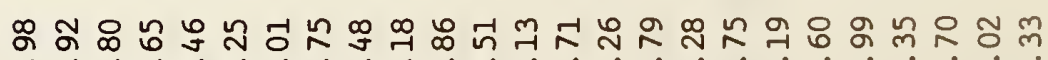

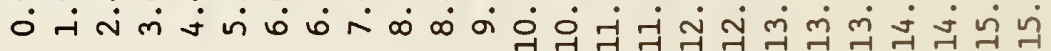

צ

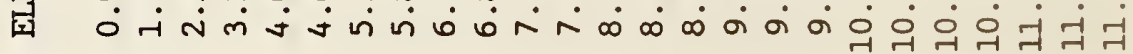




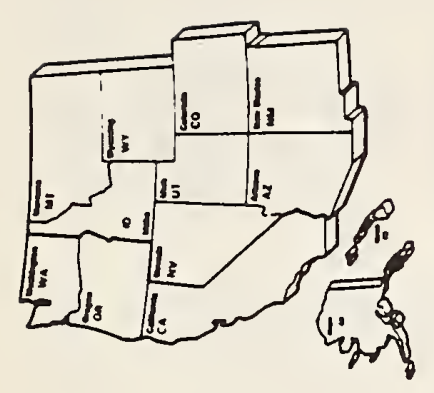

兽

氛

环 党

突国

舟

《응 웡 엉

品起谣

经吼

国卷

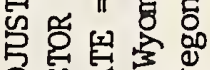

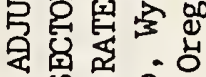

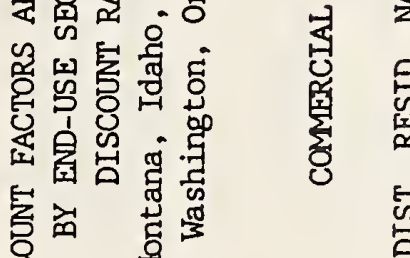

8

今

管

空

娄

貧

ปู่

ช్곡

옹

䍃 z

-

タั

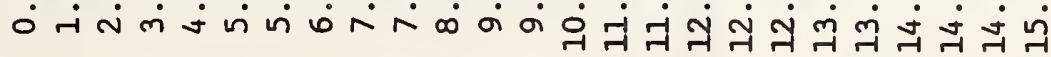

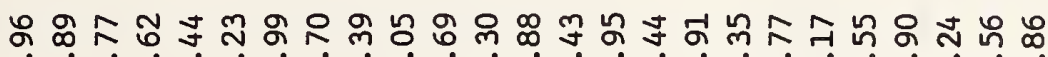
- $\dot{\sim} \dot{\sim} \dot{\sim}$ i

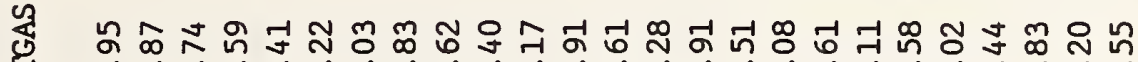

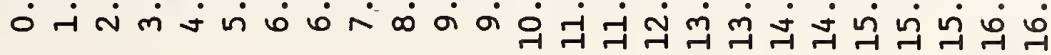

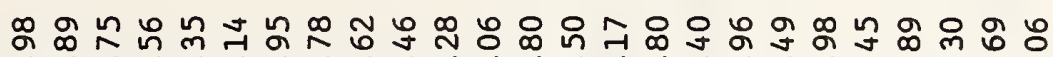

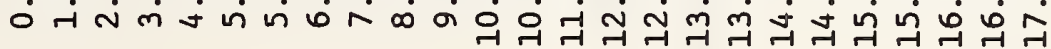

乡 -i పే

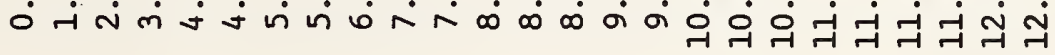

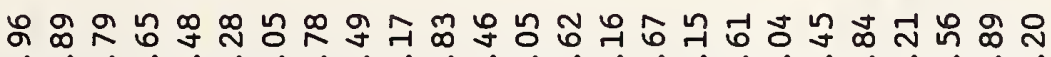

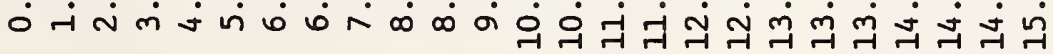

㱑 ๙

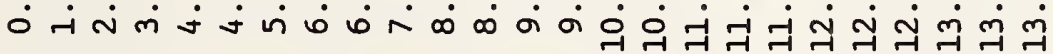

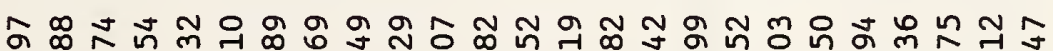
○ त

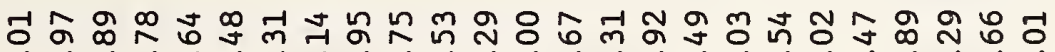
ప大

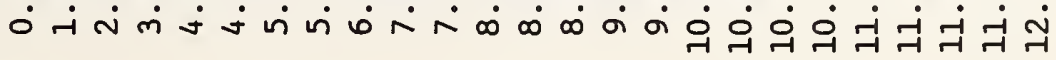

ณ - $\dot{\sim} \dot{\sim} \dot{\jmath}$ ம

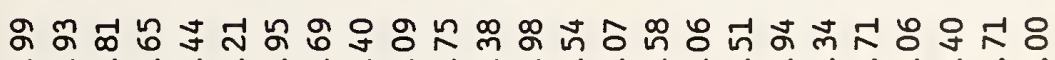

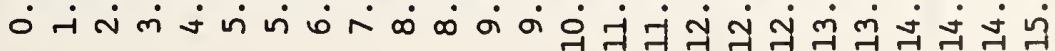
ร ภำ

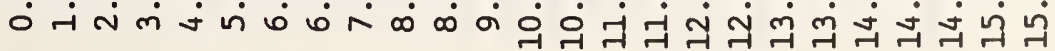

昜

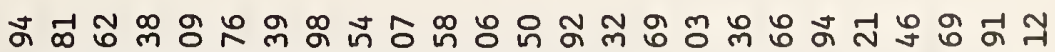
- 


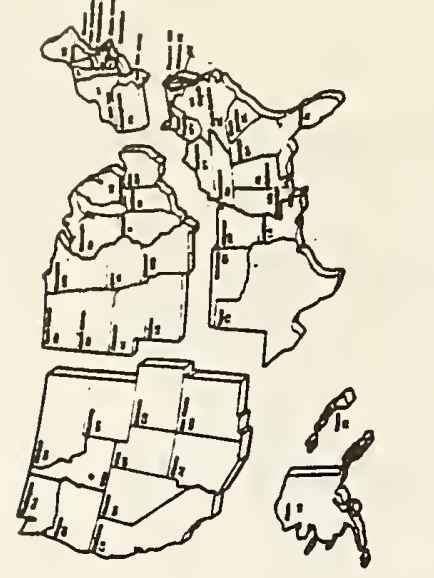

๙

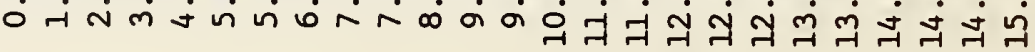

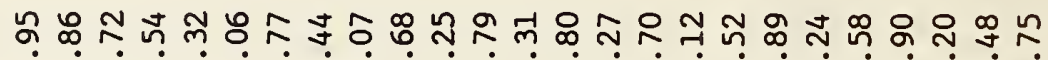

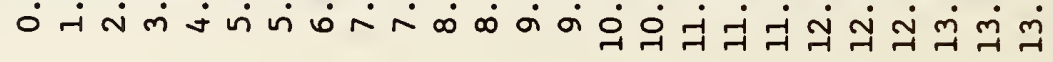
ดิ

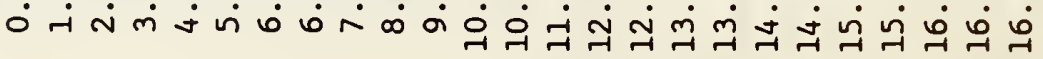

o g ๓

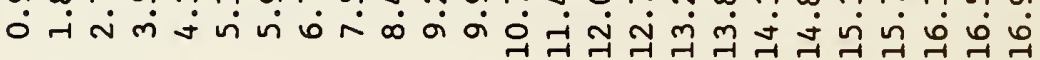

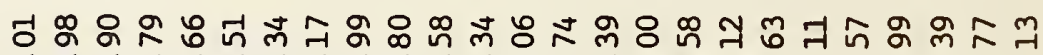

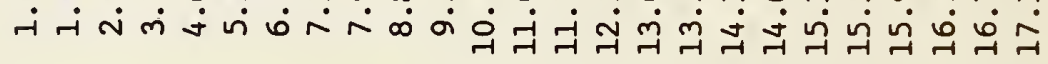
ต

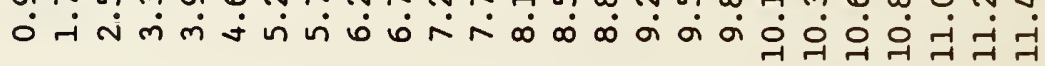

焉国

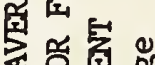

4

造运造

星是人安

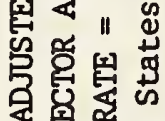

ल

窝窝

Һ

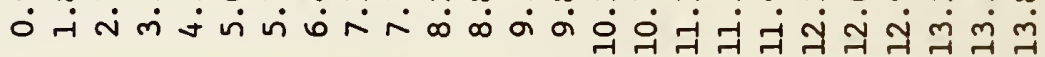
๙

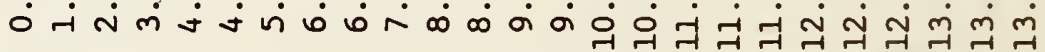

कิ

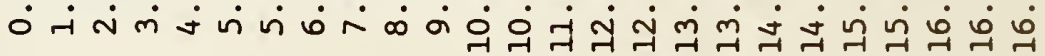

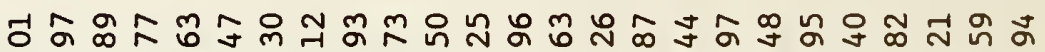

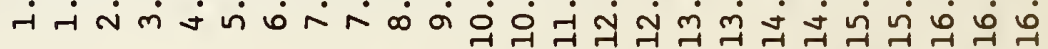

各

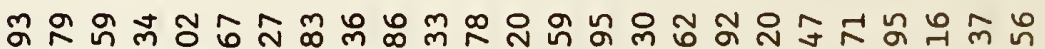

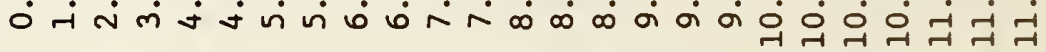

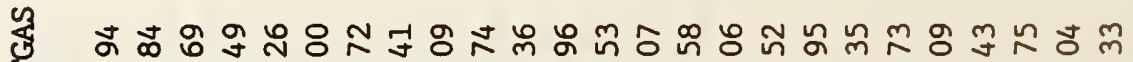
育

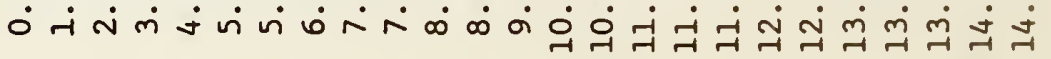
ร

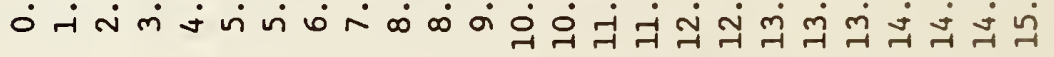
Я

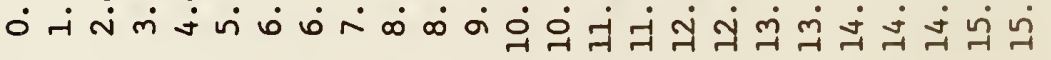

หู

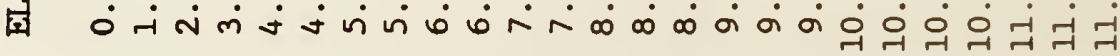




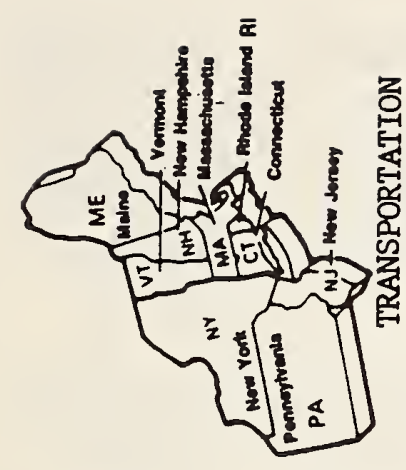

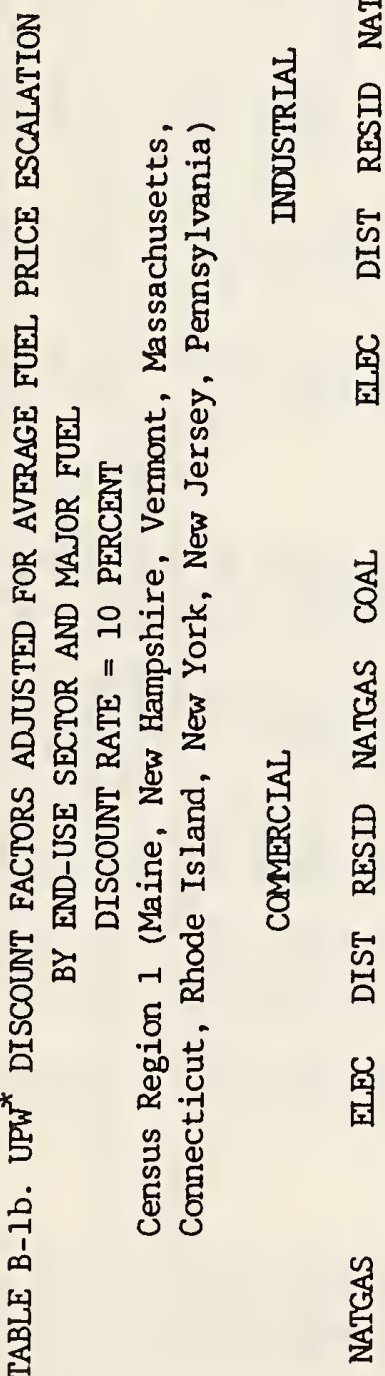

言悬

苟

䆓

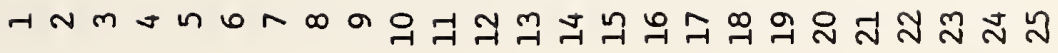

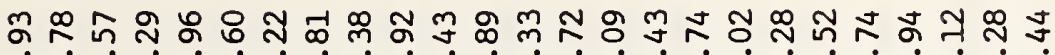

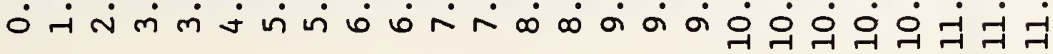

영

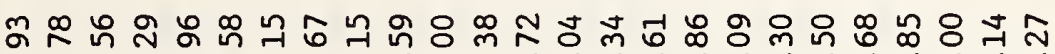
-

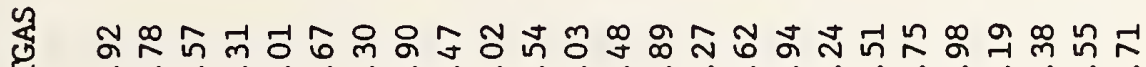
岁

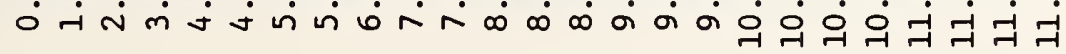

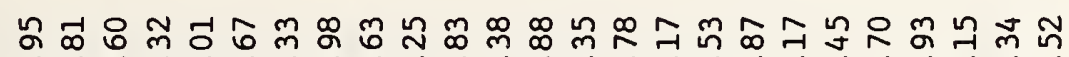

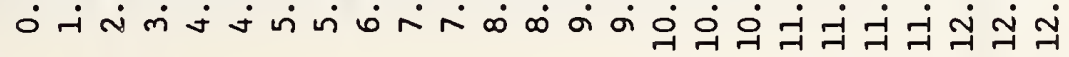

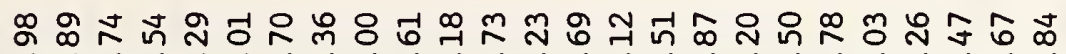

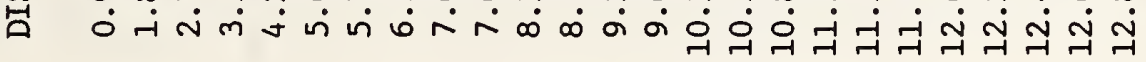

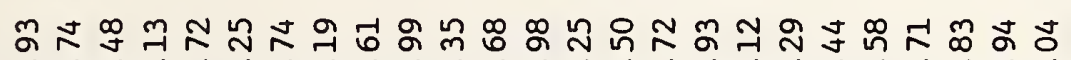

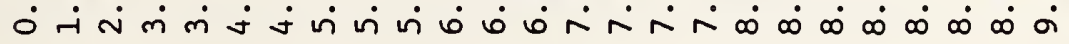

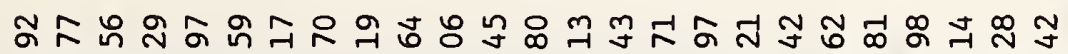

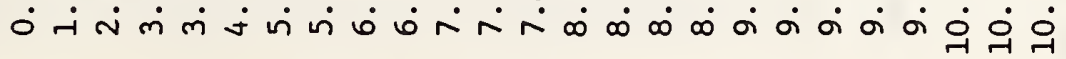
ㅊํำ กิ

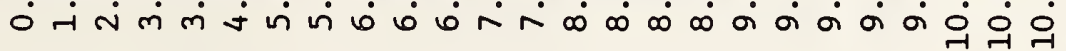

凡 요

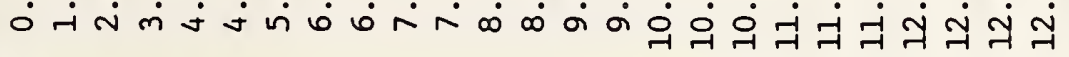

品

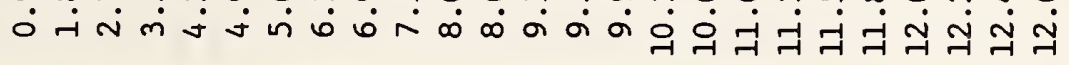

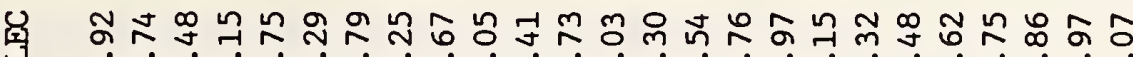

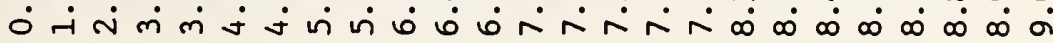

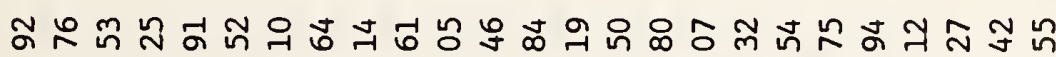

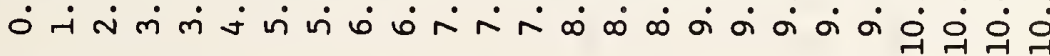

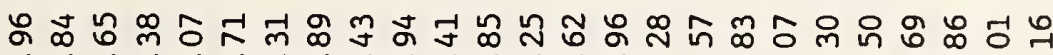

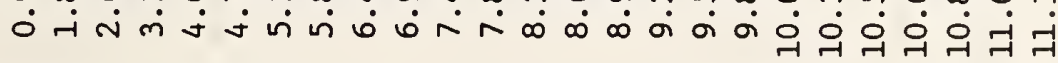

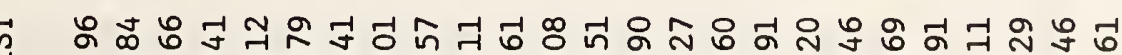

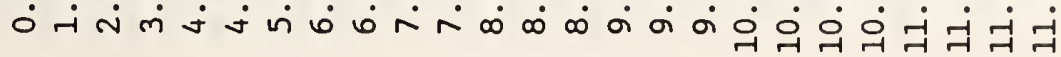

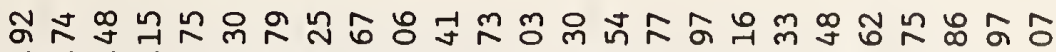

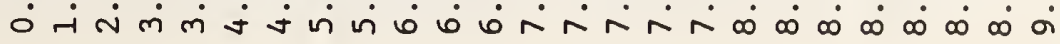

노ำ ㅇํㅇำ

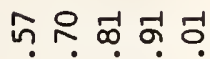
ન્ન

익 웡요웅 엉엉엉

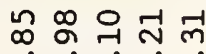

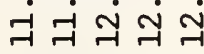

ஸ ๙

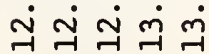

웜 $\dot{\sim} \dot{\sim} \dot{\sim} \dot{\sim} \dot{\sim} \dot{r}$

긍ำ वं वं $\underset{*}{*}$

. ڤ્山

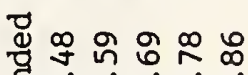

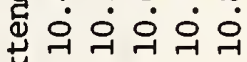
苗

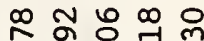
*

용요윤 눈 귀 $\ddot{\gamma} \dot{\sim} \dot{\sim} \dot{\sim}$

กำ 유 ํำ बं बं बं

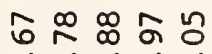
욱엉ㅇㄱ $\dot{ }$

옹ㅇㅇ 응 응 각ન ન્ન

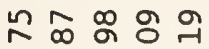
ન્ન

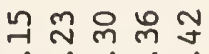
वं

ำ ำ ㅇำ 


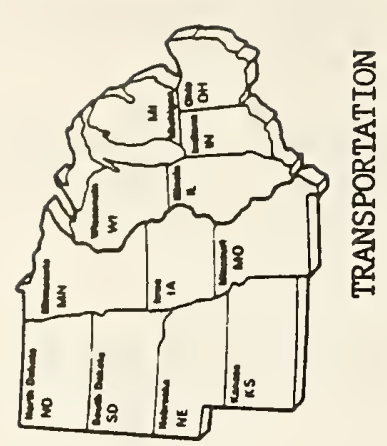

z

ᄀNmงแ

造

ตั

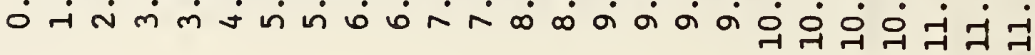

엉

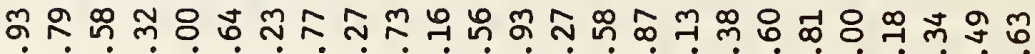

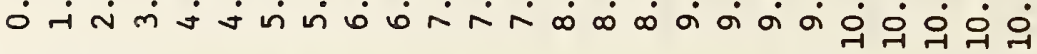

承

总

ตำ 유ำ

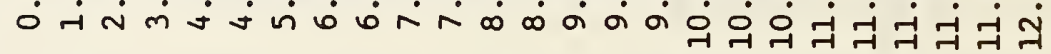

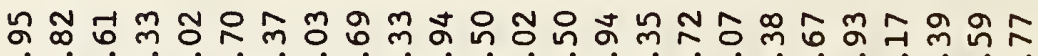

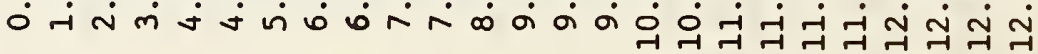

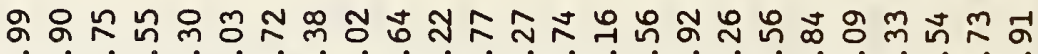

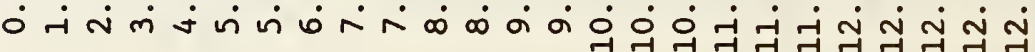

国

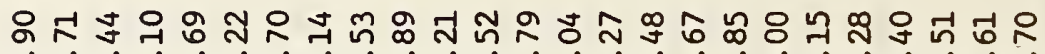

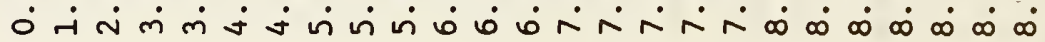

药

สำ

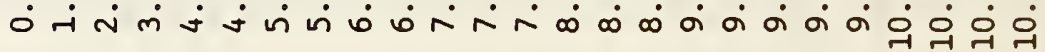

国是是

留

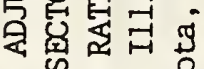

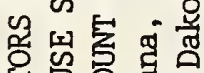

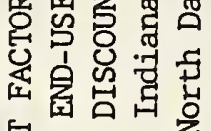

客昆

岁是

岕

Кㅊ

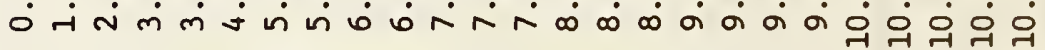

๙

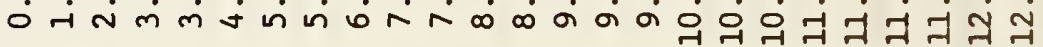

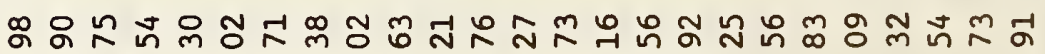
-

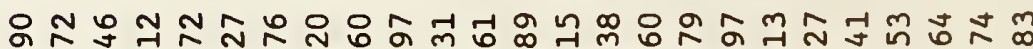

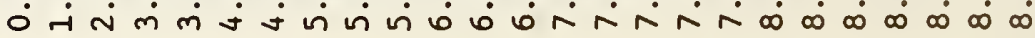

密

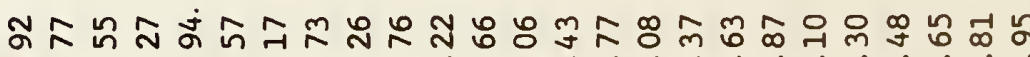

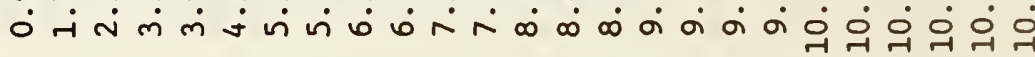

ลิ

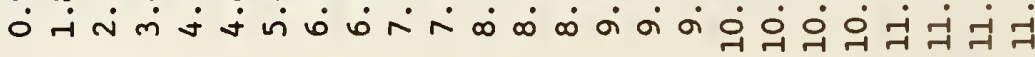

島

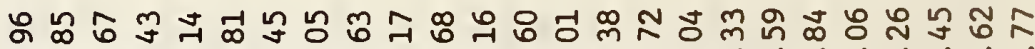

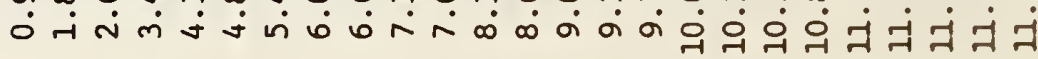

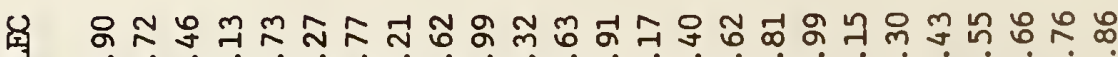
国
นัง สุ ㅇำ

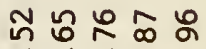
નં $\dot{-} \dot{-} ન \dot{ન}$

ํํㅇำ 웅ㅇㄱ 걱 ஸึษ

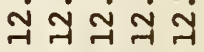
ปั ㅇํำ ผ ㄱनㄱㄹन ㅇํㄱ ๗ ले ने નેન œ $\infty \dot{\infty} \dot{\infty} \dot{\infty}$ $\underset{*}{*}$

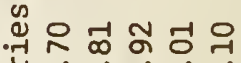

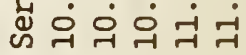
항어엉ㄱㄱ

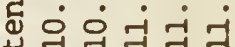
落

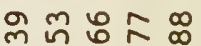
*

ธิ નું

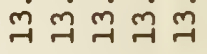

न얃ㄷㄷ $\infty \infty \dot{0} 0$ \%

등 의 응 용 ન્નુનન ન્ન

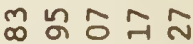
ન્ન ન્ન ન્ન สำ ำ ำ નં

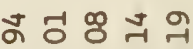
$\infty$ कं वं 

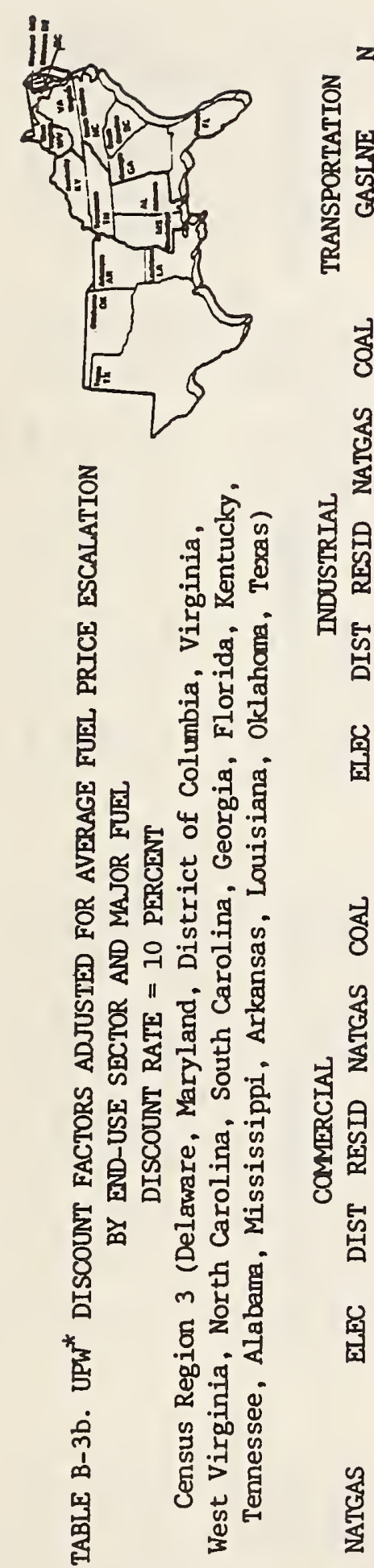

䁌总

回 경

초
8

我 要 $z$ $-1$

\section{ศิ -} ० $\dot{-1}$

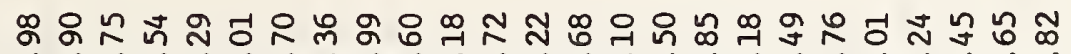

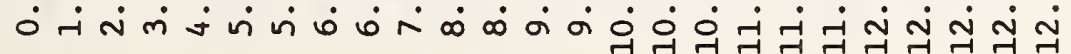

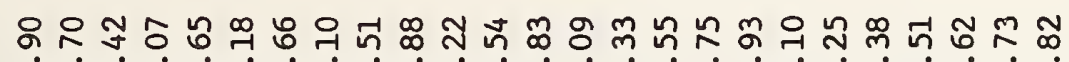

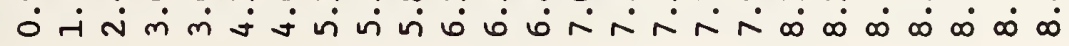

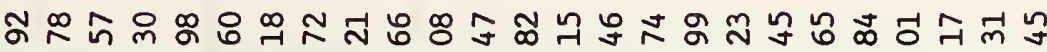

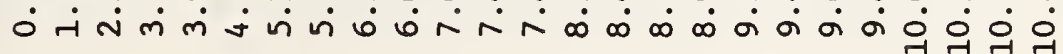

สำฟ -

็ู ำ

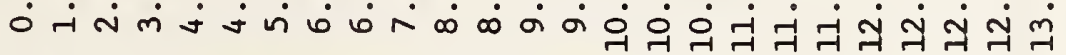

品 0

어워 융ㄱำ

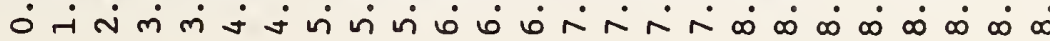

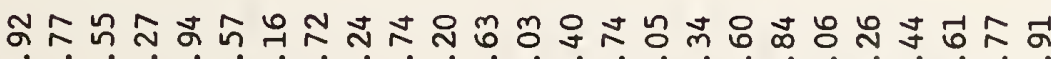
-

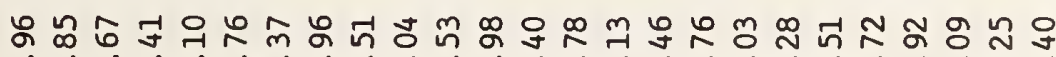

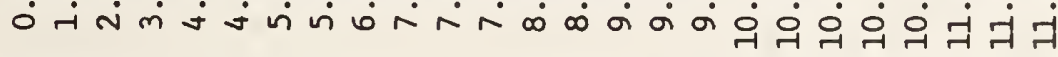

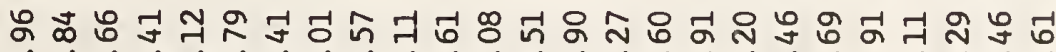

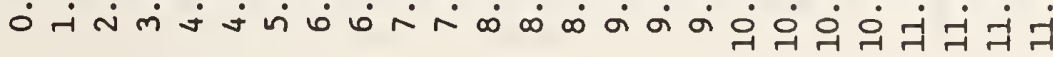

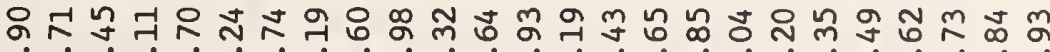

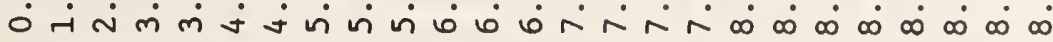

ำ 옹 ㅇำ

ஸึ

ન્ન ન્ન ન ન ન

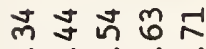
엉어웅웅

สี่ $\dot{\vec{r}} \ddot{\sim} \dot{\vec{r}} \ddot{\sim} \dot{\sim}$

응으ㅇㅠㅜ

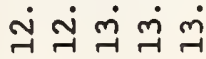

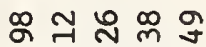
굴 $\ddot{\sim} \dot{\sim} \dot{\sim} \dot{\sim}$

용읟ㄱㄱ $\infty \dot{\infty} \dot{\sigma} \dot{\sigma}$ $\underset{*}{*}$

. ํำ

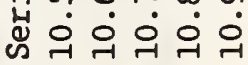
잉

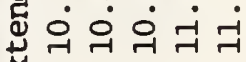
苗

구 क ำ * $\ddot{\sim} \dot{\sim} \ddot{r} \ddot{r} \dot{r}$

ดㄱㄱㄴㅇ $\ddot{\sim} \dot{\sim} \dot{\sim} \dot{\sim} \dot{\sim} \dot{\sim}$ 응 궁ำ

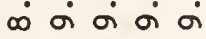

ตูก กิ้ ન્નુનન ન્ન

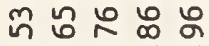

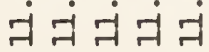

ก નં

덩옹 누 추 वं वं

ำ ลั ำ 


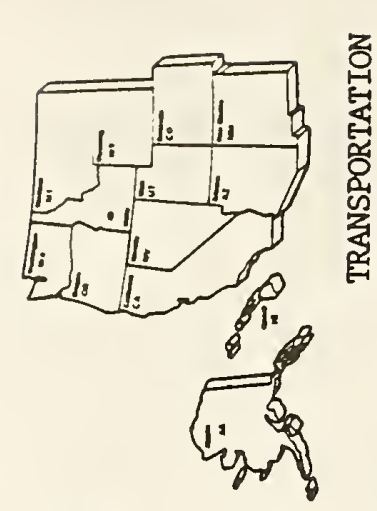

2

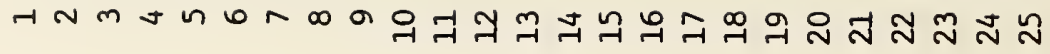

密

ตำก ลิ

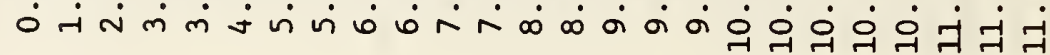

영

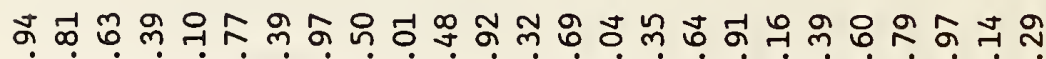
-

空

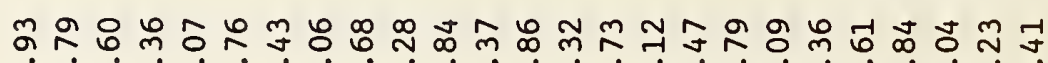
-

ૂ

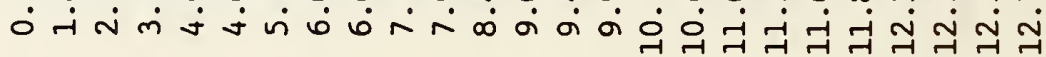

岕

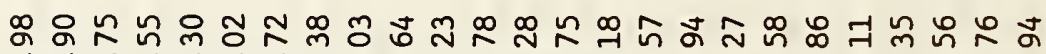
-

㐿

бำ

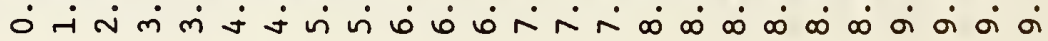
县웅

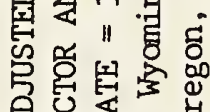

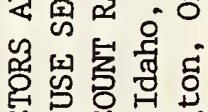

论

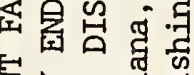

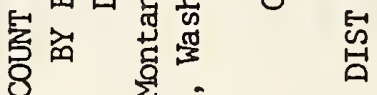

* $\quad$ \&

宾

定

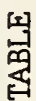

善
봉

宾

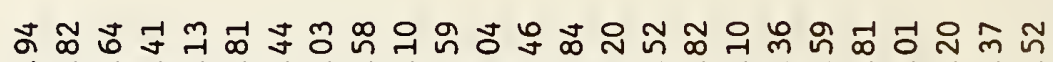

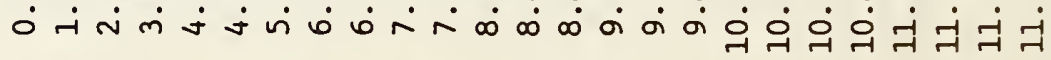

ไ్

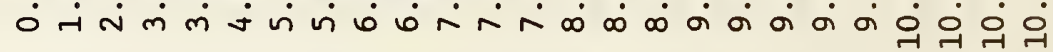

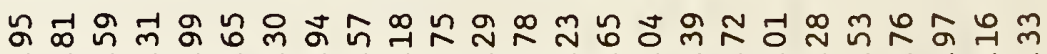

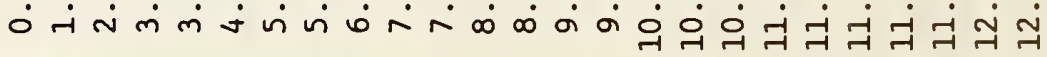
品 ๙

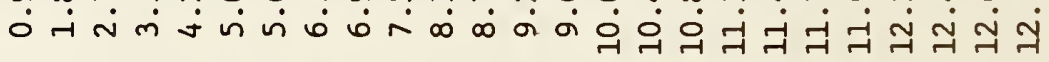
万ூก

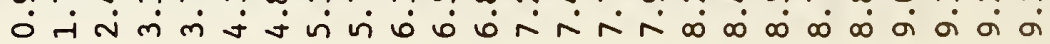

द्य

葛吕

$z$
獣

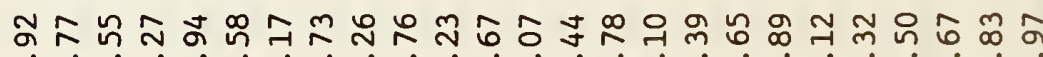
○

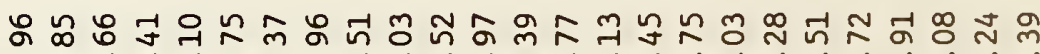

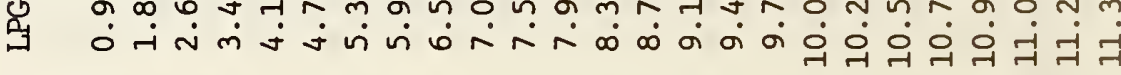
ตํำ

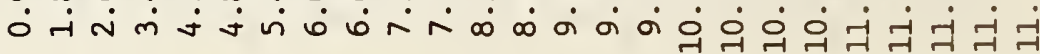

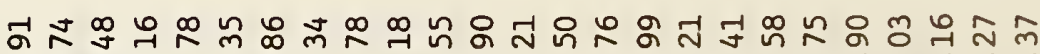

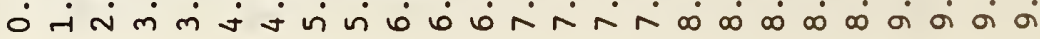

ํำ 옹 유

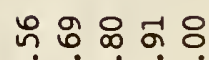
ન્ન ન્ન ન્ન ન્ન

งิ กี ન્નું ન્ન ન્ન

늉요 용 भૈ ศี નี નี

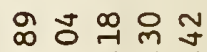

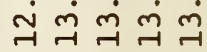

ㅇํㅇ กิ ㅇำ

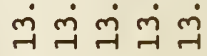

กินก बं बं $*$

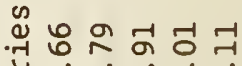
ڤँ ન્ન ન્ન ન્ન 국 평용ㅇㅇㅇ

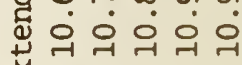
苟

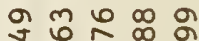

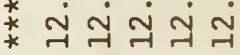

๓ ูำ ते $\dot{\sim} \dot{\sim} \dot{\sim} \dot{\sim}$ ๆ $n 80 \hat{n}$ कं वं

욱 ㅊำ ำ ำ ન્નુંનન્ન กี้ ฐ๊ ณ ન્નનનનનન્ન ตู ન่ สี่ สี่ ชี ช้ ฟูง कं वं का

ำ ำ ㅇํำ 


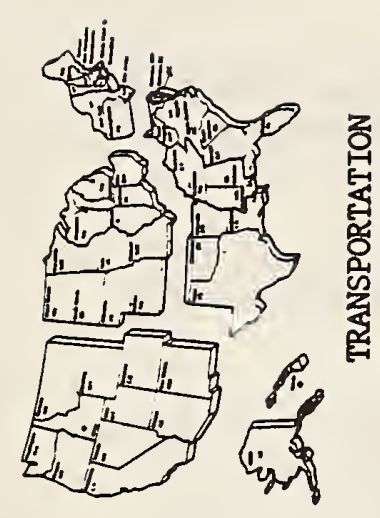

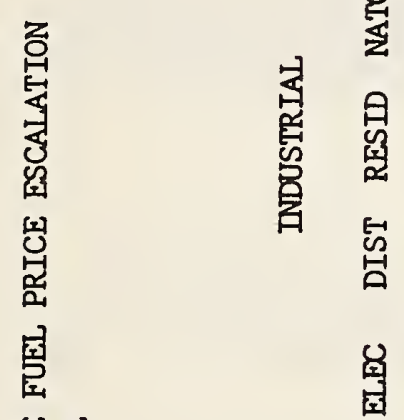

罂宝

究息。

ช零舀

웅운

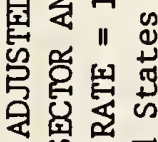

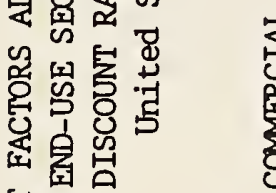

家品

8

㟔

宩

ถุ่

ต

됩

蛋

총

究

星

点

堹

胥

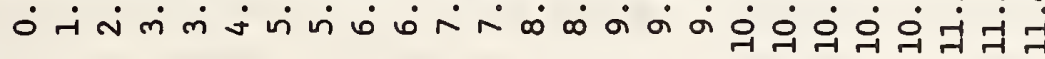

엉

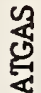

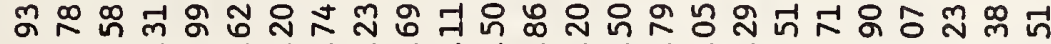

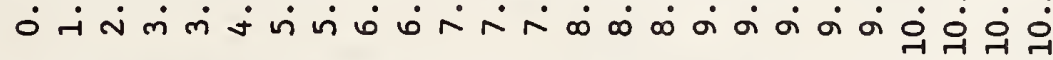

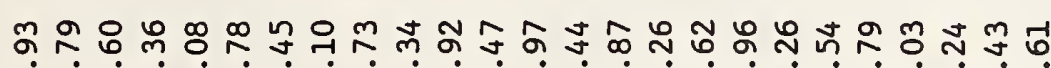

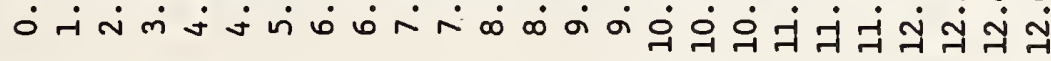

๙

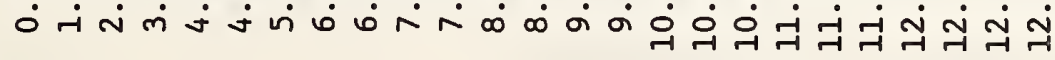

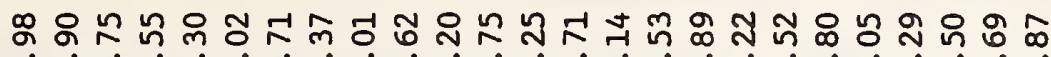

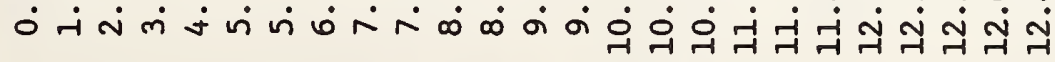

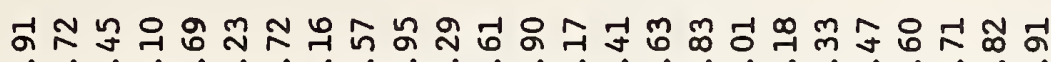

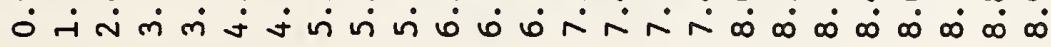

๓

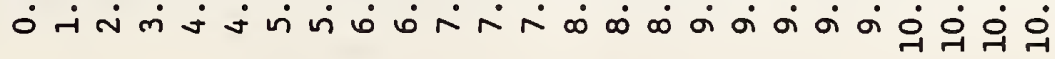

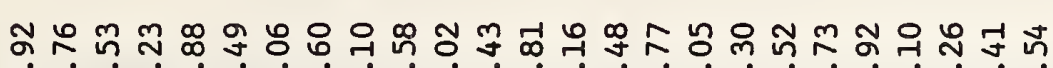

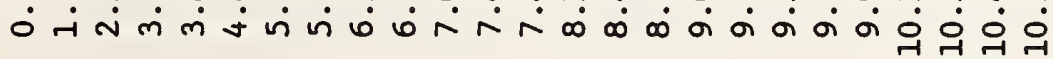

๙ุ.

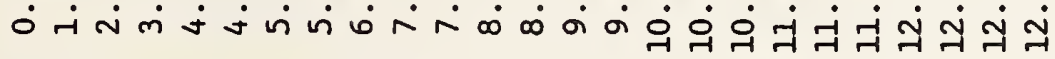

の ฌ ல

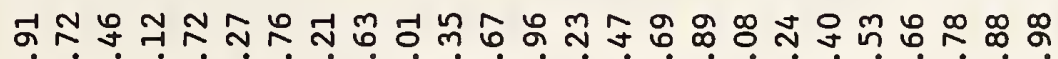

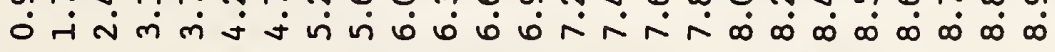

毼

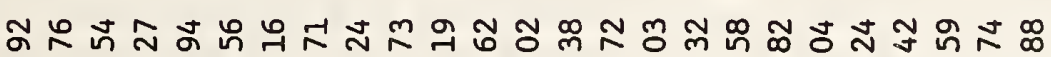

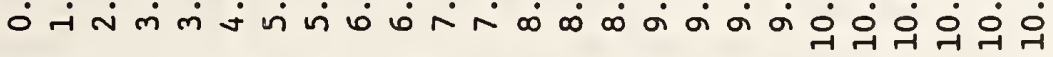

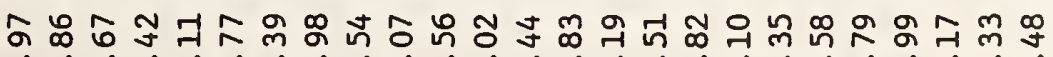

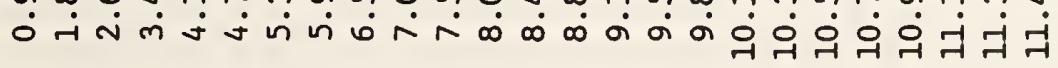

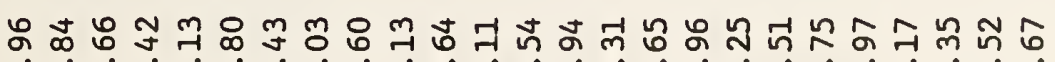

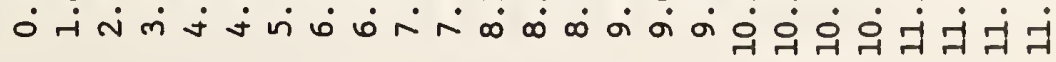

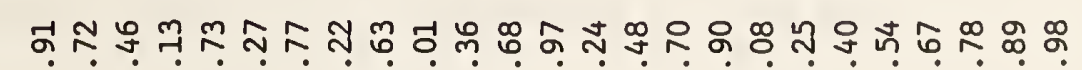

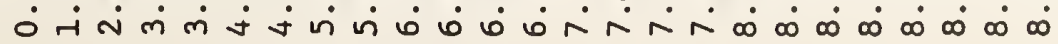

ํํำㅇํ요

นึํㅛㅇ용요 ન-ં ન્-

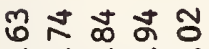
$\dot{\circ} \dot{\circ} \dot{\circ} \dot{\circ} \dot{-}$

처ำ ํํำ ㄱํㄱํ भु

응유요

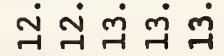

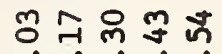

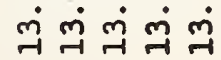
응규유 웅 कं

엇오 岁远酧

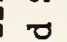

ชัญ ํㅜ 过近近近 血

응요에 * 잉 누 옹 ㄱํㄱ $\dot{7} \dot{न} \dot{~}$ 넝오 बं

ริำส สิ ซี ન્ંનંન્ન ન્ન

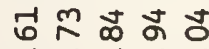
નં નં નં નં

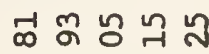
નં નં જี ธำสำ कं वं कं

놏 옹요 
1988 AVERAGE FUEL PRICES, PROJECTED AVERAGE FUEL PRICE INDICES, AND PROJECTED AVERAGE FUEL PRICE ESCALATION RATES FOR FEDERAL USE (INDICES AND ESCALATION RATES EXCLUDE GENERAL PRICE INFLATION)

Table C presents average 1988 fuel prices for the 4 Census regions and for the United States. Use these prices as default values for 1988 fuel prices only if you do not know actual fuel prices.

Tables $\mathrm{Ca}-1$ through $\mathrm{Ca}-5$ of this section present projected average fuel price indices for the 4 Census regions and for the United States. These are multipliers which when applied to the 1988 prices provide estimates of the corresponding future-year prices in 1988 dollars. Note that the resulting price estimates are in constant dollars, exclusive of general price inflation. Constant dollar prices are needed when discounting is performed with discount rates which do not include general price inflation.

Example of How to Use the Indices:

To estimate the price of industrial steam coal in year 2005 in Connecticut, go to table $\mathrm{Ca}-1$, find the year 2005 index for industrial steam coal (1.27). and multiply by the 1988 price for industrial steam coal in Connecticut. The result will be given in 1988 dollars.

Tables $\mathrm{Cb}-1$ through $\mathrm{Cb}-5$ present the projected average fuel price escalation rates (percentage change compounded annually) for six selected periods from 1988 to 2013 for the 4 Census regions and for the United States. Note that these are "real" rates exclusive of general price inflation. Their use results in prices expressed in constant dollars.

The escalation rates consolidate the information provided by the indices in the $\mathrm{Ca}$ tables so that trends in projected price changes can be seen at a glance. They are provided primarily to accommodate those who use computer programs which require escalation rates as inputs.

Unless there is a compelling reason to use escalation rates, it is recommended that you use the indices in the $\mathrm{Ca}$ tables to estimate future-year energy prices, since the indices include year-to-year information rather than averages over a number of years, and are easier to use.

\section{Example of How to Use the Escalation Rates:}

To estimate the price of residential distillate in $1993\left(\mathrm{p}_{93}\right)$ in Wyoming using the escalation rates, go to table $\mathrm{Cb}-4$ and find the 1988-1990 and 1990-1995 escalation rates for residential distillate $(3.68$ and 2.98 per year, respectively). Assuming the actual 1988 price of residential distillate is unknown, go to table $C$ and find the default 1988 price $\left(p_{88}\right)$ for Census region $4(\$ 6.67 / \mathrm{million} B(u)$. Enter these values into the following formula and solve for the 1993 energy price (stated in 1988 dollars):

$$
\begin{aligned}
P_{93} & =p_{88} \times\left(1+e_{1}\right)_{1}^{k} \times\left(1+e_{2}\right)_{2}^{k}, \\
& =\$ 6.67 \times(1+0.036)^{2} \times(1+0.029)^{3}=\$ 7.80
\end{aligned}
$$


where $e_{i}=$ Annual compound escalation rate for period $i$ from the $\mathrm{Cb}$ tables (in decimal form); and

$k_{1}=$ Number of years over which escalation rate $e_{1}$ occurs.

For further explanation of how to use these tables, see NBS Handbook 135 , appendices $C$ and $G$.

Note: The data in the tables which follow are now reported by 4 Census regions. Prior to the 1988 edition, regional data were presented by 10 DoE regions. Figure B-1 presents a map showing the states corresponding to the 4 Census regions. The Census regions do not include American Samoa, Canal Zone, Guam, Puerto Rico, Trust Territory of the Pacific Islands, or the Virgin Islands. Analysts of Federal projects in these areas should use data which are "reasonable under the circumstances," and may refer to the U.S. average data for guidance. 


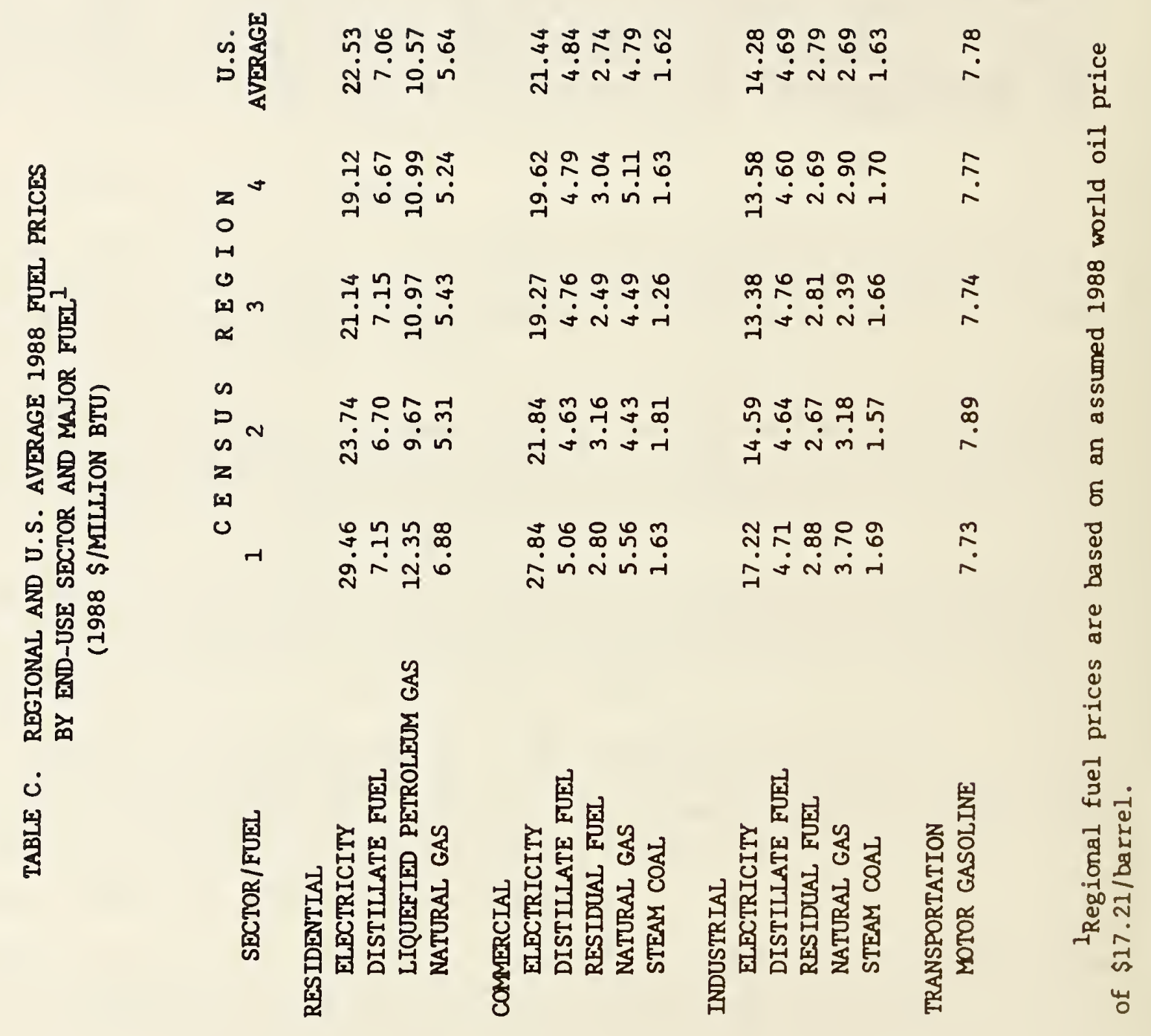





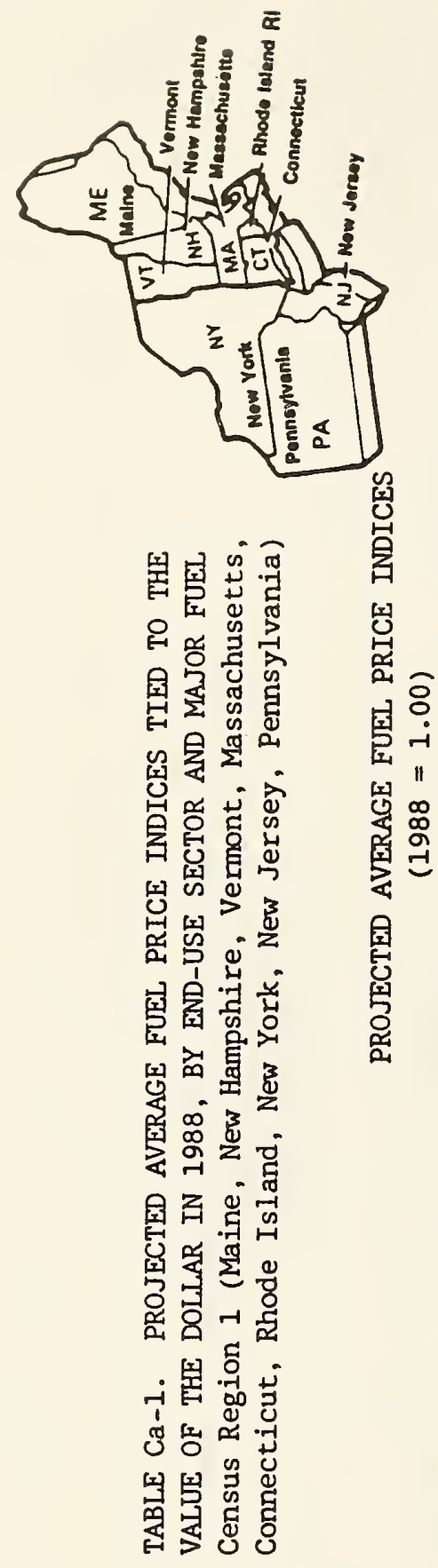

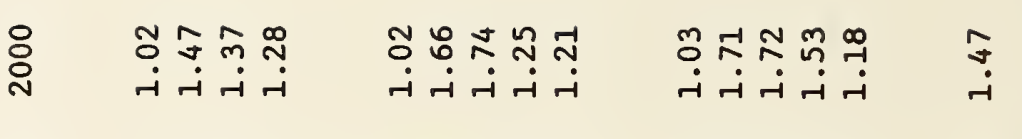

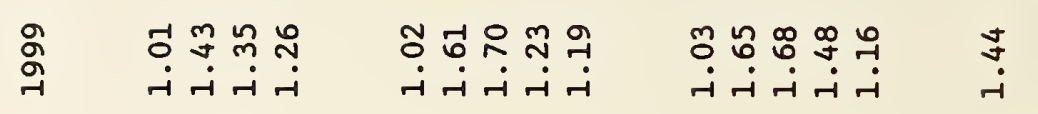

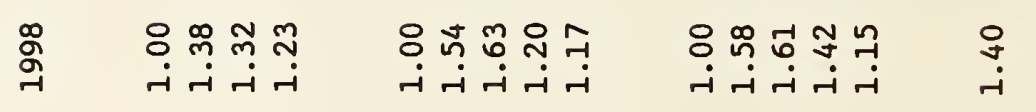

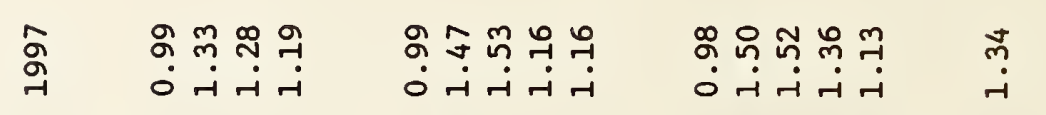

๕

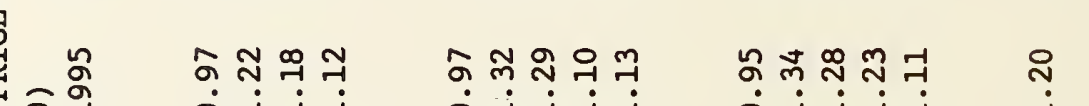

o

긍

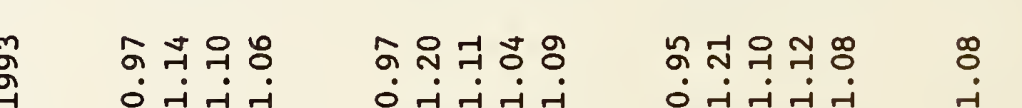

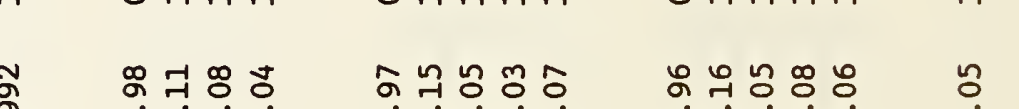

gू कून

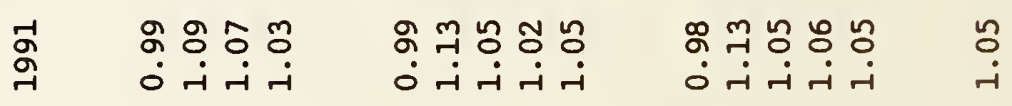

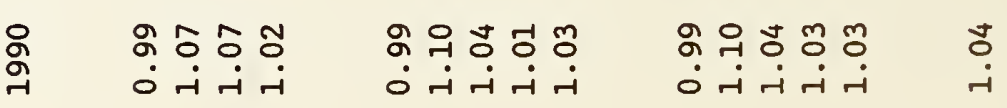

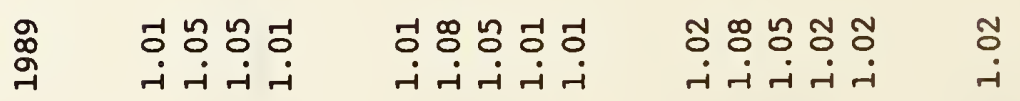

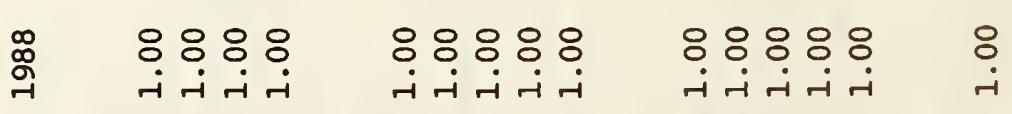

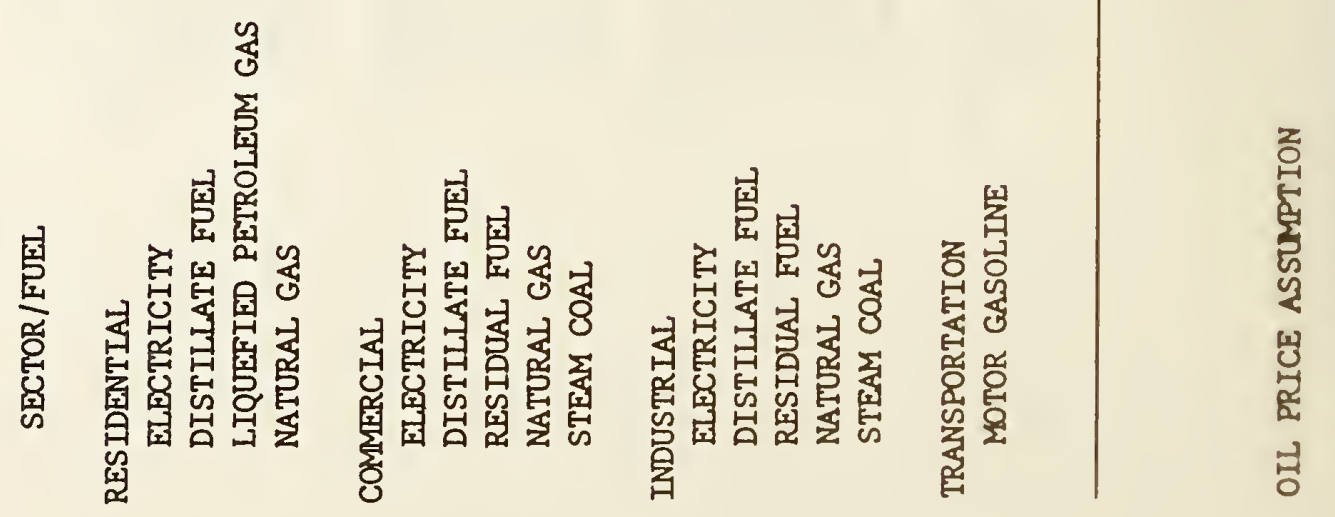




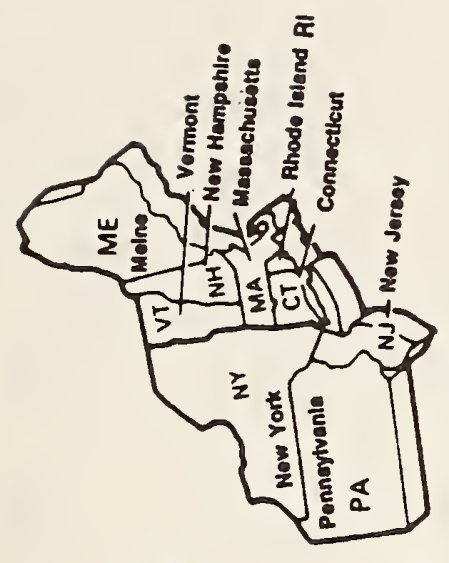

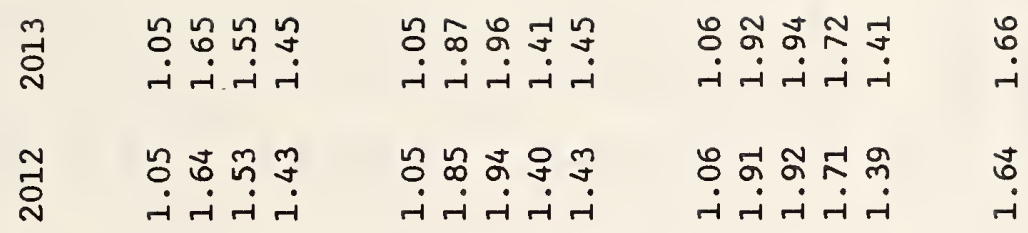

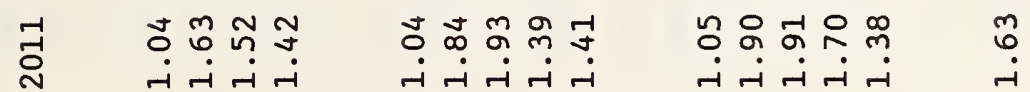

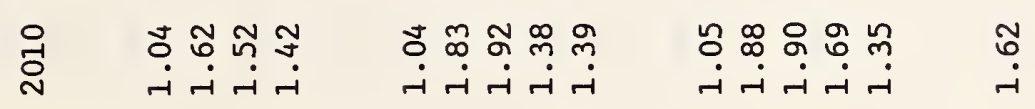

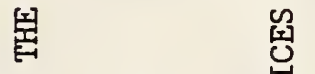

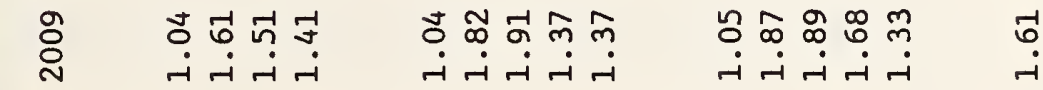

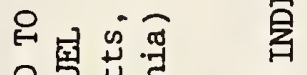

思

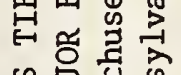

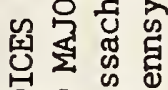

羊是罢路.

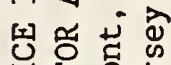

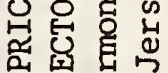

牙约

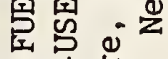

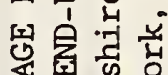

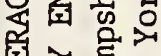

密空总

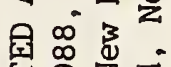

界栾 $\approx$

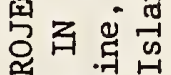

谷

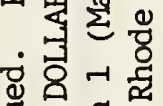

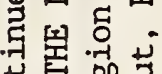

空

国

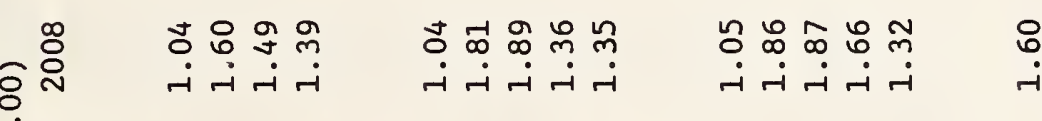

")

宽

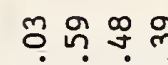

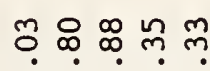

능 요용요

-

$\sum_{\infty}^{\infty}$

娄

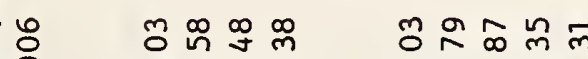

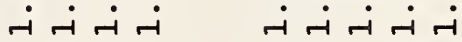

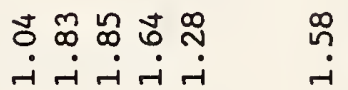

ฟ $-1-1$

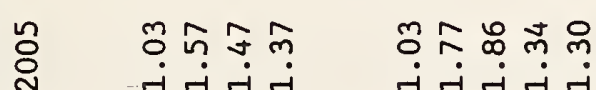

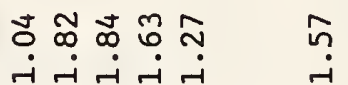

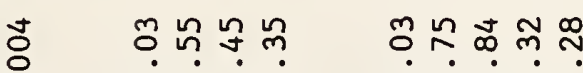

ธุำ

용

ธิ กิ ษ

मำ

mํํ에

-

on

옹

กิ ที่

iभ

-i

ธัำำกำ กิ

मં

ㅇำ

용

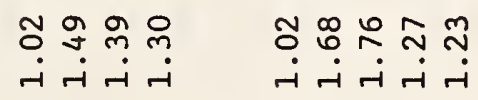

ตำกำกั่

$\underset{\infty}{\infty}$

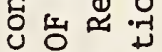

谓哭苋

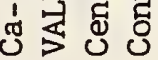

-ં

mำำำกำ ㅇำ

$\stackrel{\infty}{\infty}$

红
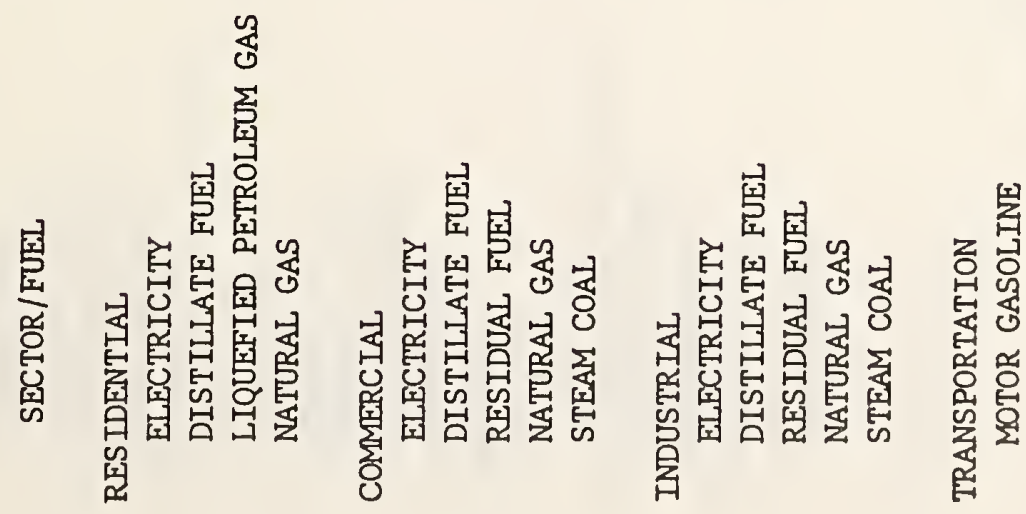

z్. 


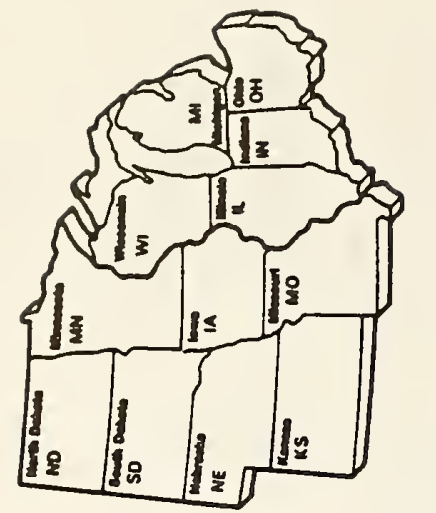

号

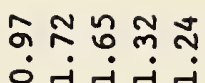

ถูกำก

$\stackrel{?}{\rightarrow+}$

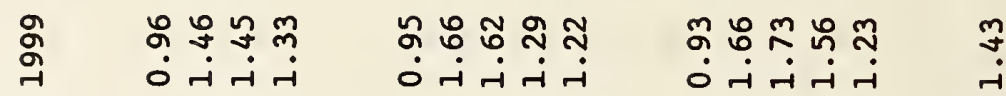

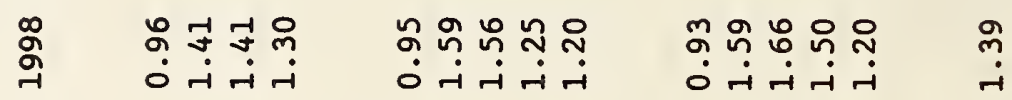

ڤે $\quad$ ŏ

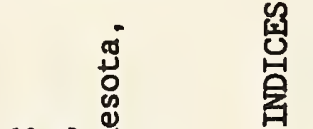

鯯

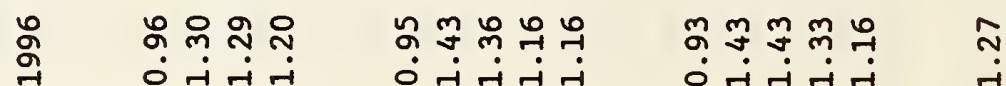

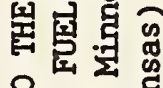

웅 द

园零密

经是需

进骂

唇嵒

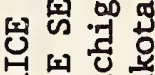

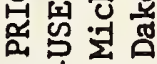

居最品

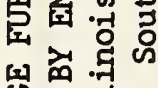

垈留

这

त腾

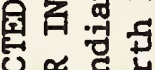

造䍃

电

응

占

ณ

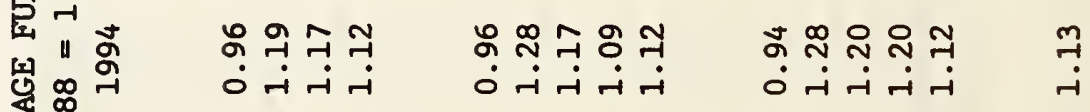

目

gू $\quad$ ă $\tilde{r}$

8

$\dot{0} \dot{i} \dot{-i}$

ลำํํㅇ웅

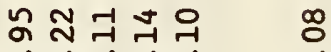

જૂ

ठं

ठमन

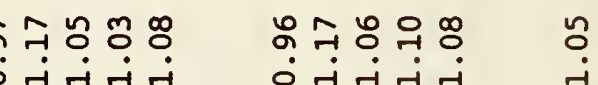

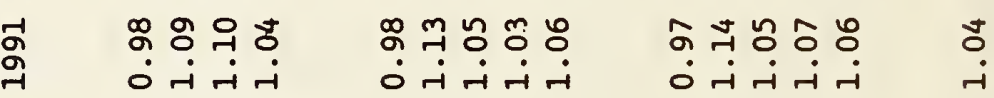

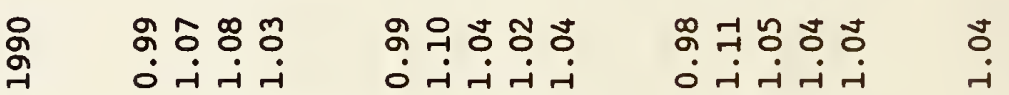

范

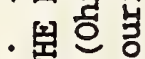
)

ช่

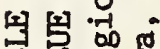

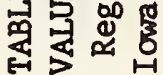

总

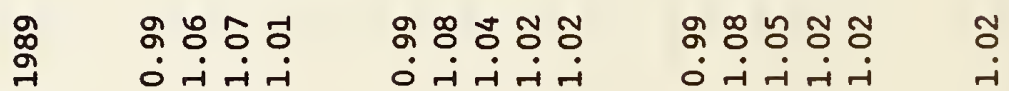

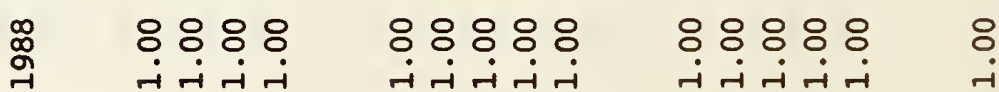
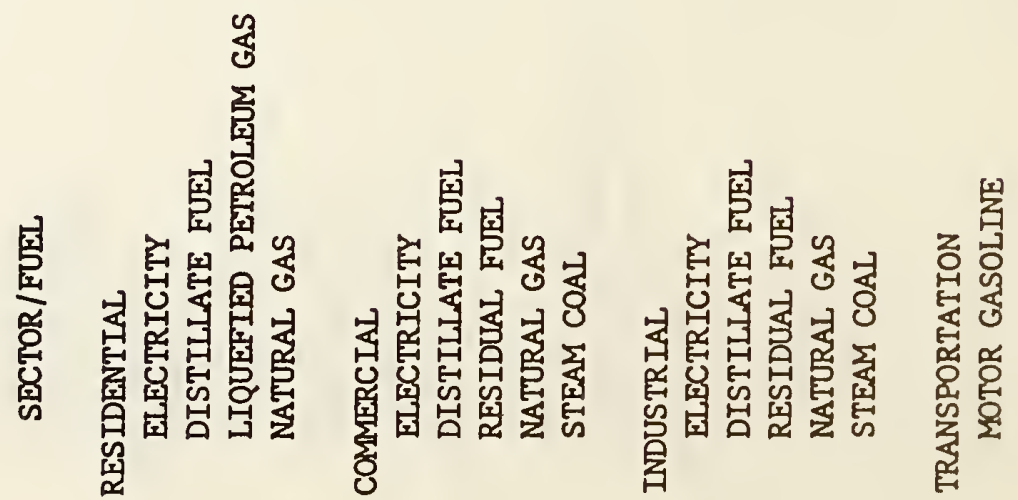


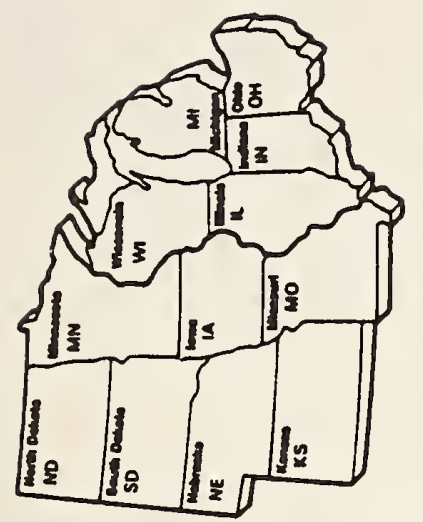

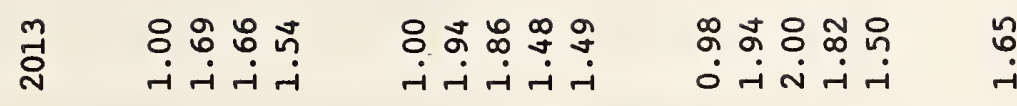

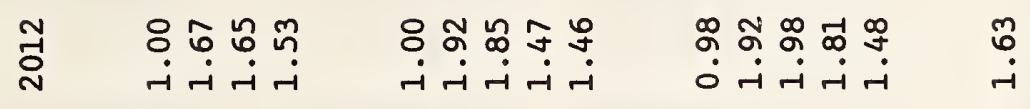

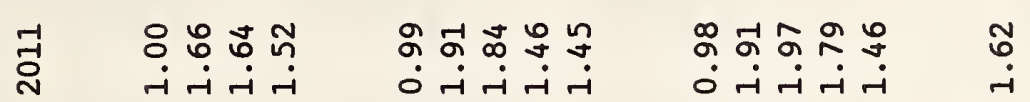

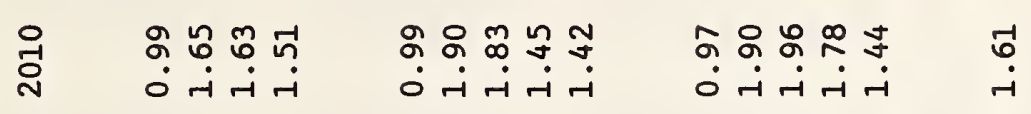

웛 苋

올

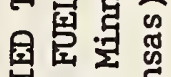

量

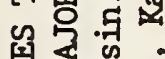

는

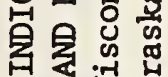

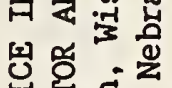

总兽

武界范

宦骂 둴형

品定

禺商哭

要离.

国表哭

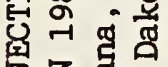

家㝵

婙落

i

可言

落N

客岁 동

罗要

睤
畐

o

왼

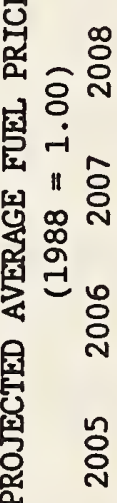

ญ̊

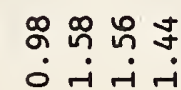

ㅇํำ ํํำ

○ُ

कำㅇำ

نَ

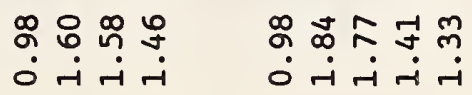

ठં

ลำ ผำำ

نं

ठं

$\stackrel{8}{\circ}$

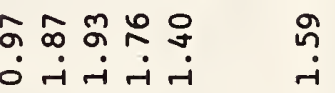

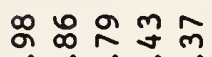

ठં नं नें

ลํํำ ณ

ठં

ֻึ

등ㅇㅇ요유

ठં नं नं

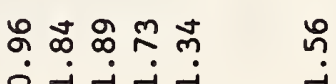

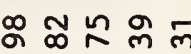

نَ

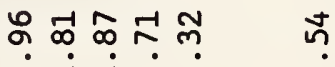

ֻั

ตั กำ กี ษ

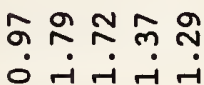

옹무용

० न न न

กี่

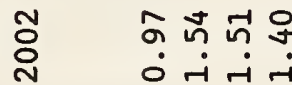

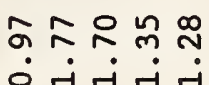

ํำ윰ำ

¿ั่

ڤิ กี้

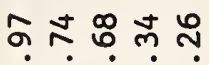
ठ नं नं

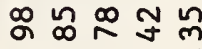

$\hat{o}$

ำ

ปั

ัั

包

월 영

렁 नं ळ

곯

20월

园 ने

名

옴

๙ั

ต

옥

$\underset{\infty}{\infty}$

$\infty$
$\infty$
$-i$

国 葟密

官国

宝

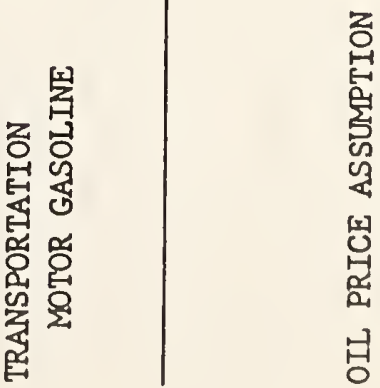




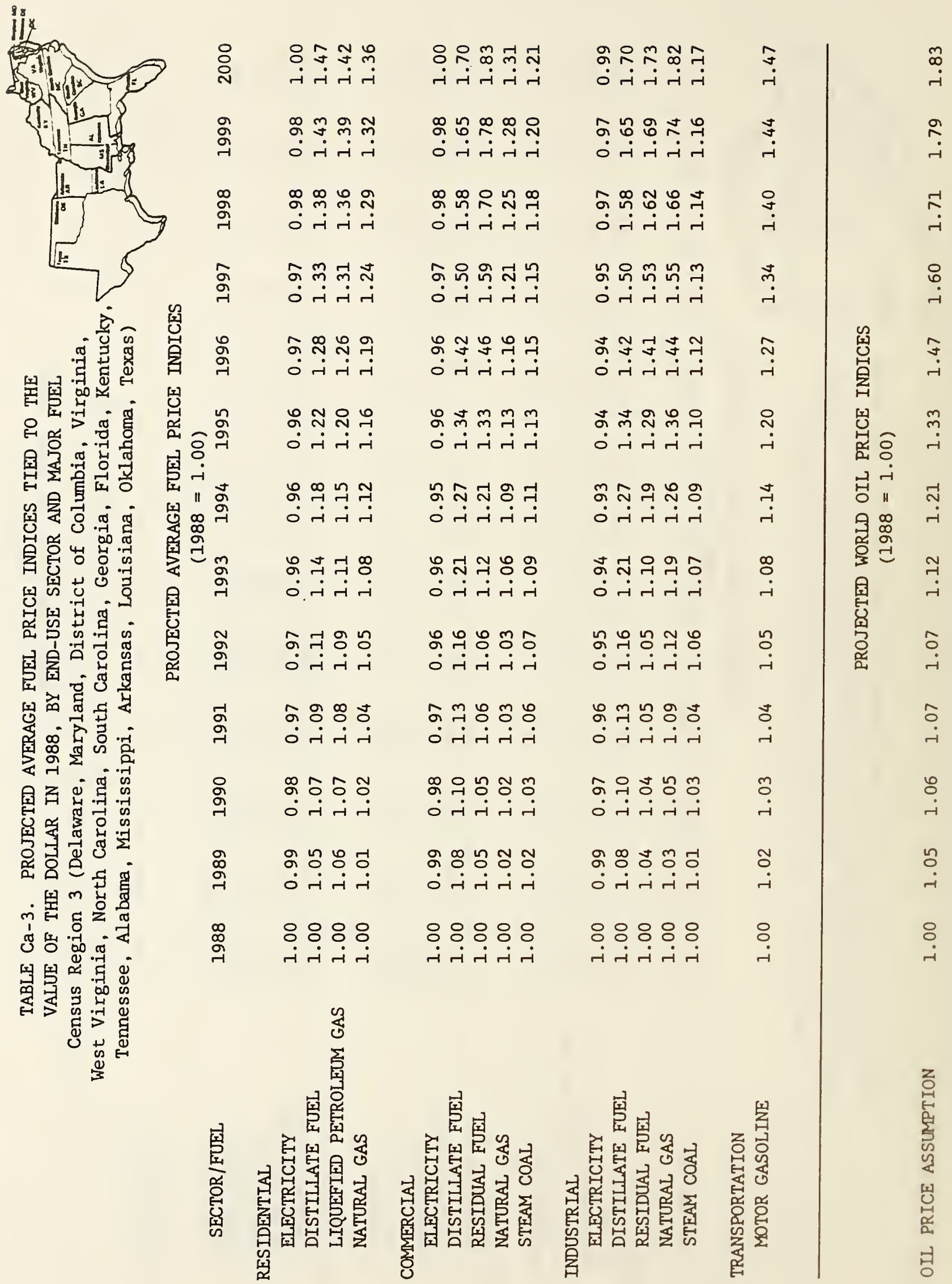


กิ่

$\hat{\circ}$

I.

సี้

$\stackrel{n}{\circ}$

?

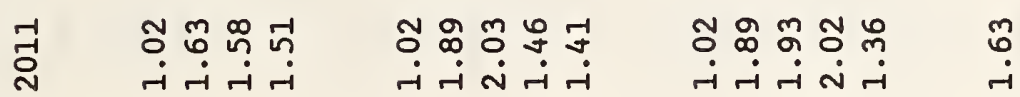

$\stackrel{4}{0}$

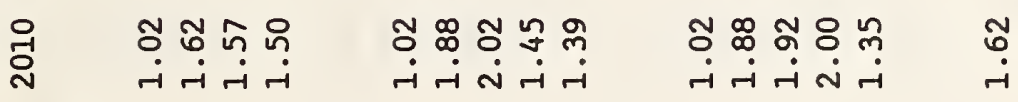

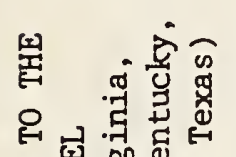

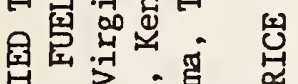

क

ชั

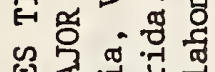

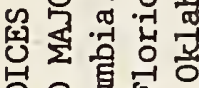

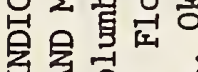

存

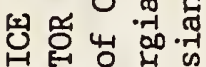

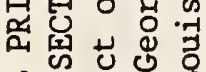

国 ब些

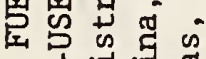

琽合年

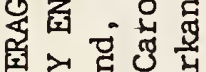

窟

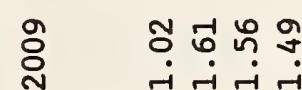

ऽ

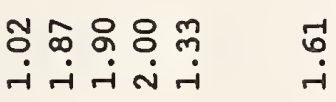

造

垴范

नं नं

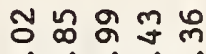

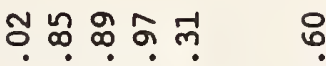

공 웅

용

"

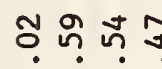

-i $\dot{-i} \dot{-i}$

$\dot{-i} \dot{-i} \dot{-i}$

ᄃ)

స઼

-i $\dot{-i} \dot{-}$ -

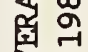

园

○)

$-m \wedge-1$

- $\dot{-1}$ -

-

โூ

-i $\dot{-1} \dot{-}$

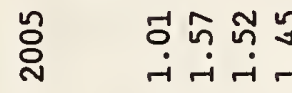

ㄷํㅇ ๙

નુ ભ

응 ㄷำ

당 ๙

य

园

खै न 2 क

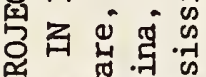

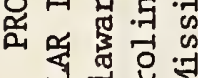

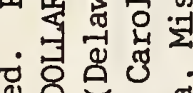

영워

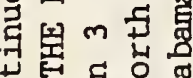

岂

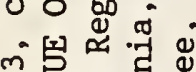

के

ख़่

艩

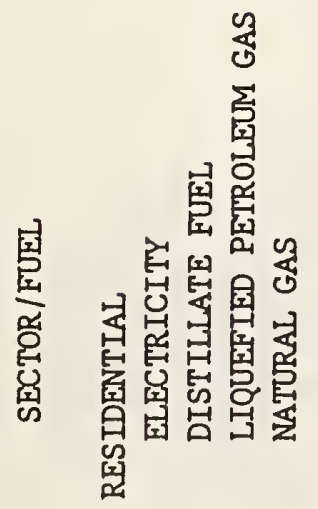

武国

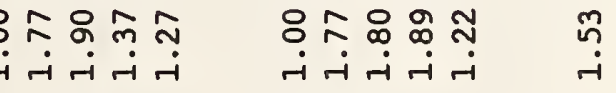

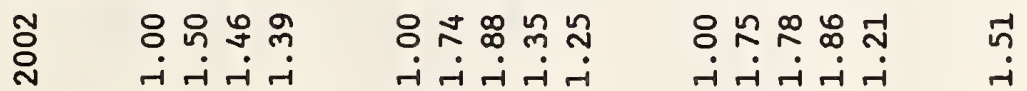

ㄷํㅇำ

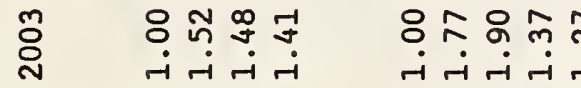

๙ุ

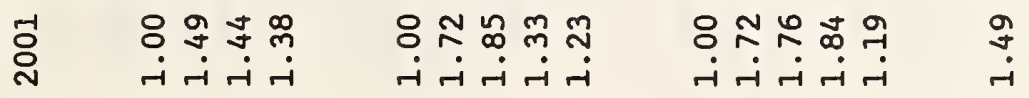

$\infty$

울 


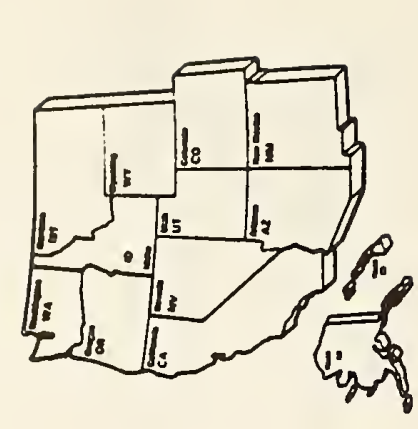

i

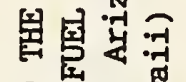

웁 ㅇํㅇ

즐

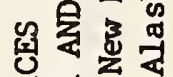

的知

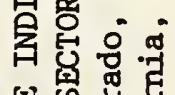

品界器

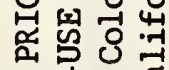

숩.

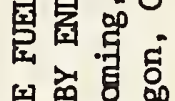

웡

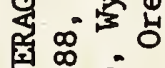

द 品 ह

目军票茄

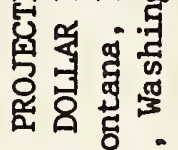

. 볼

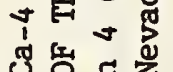

궁 5

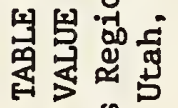

马

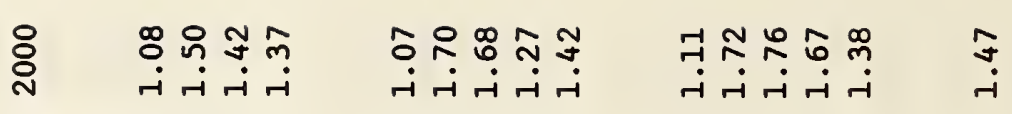

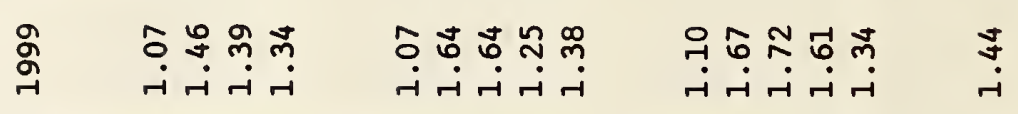

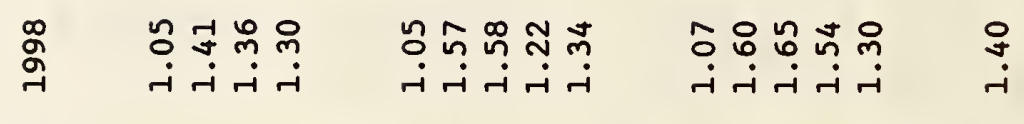

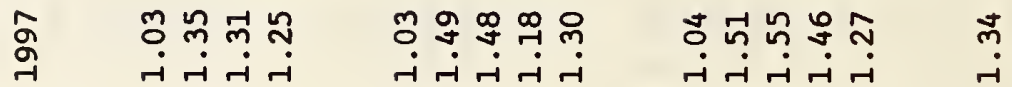

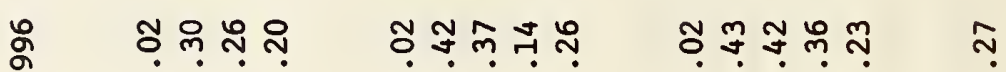

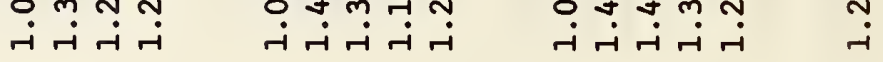

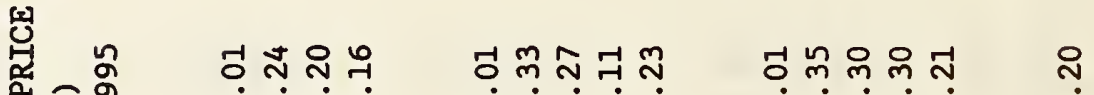

ठิ의

佂

웠

遥 总

度

gू

๙ั

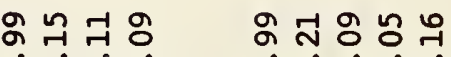

ภิ สี

ن

0

\&

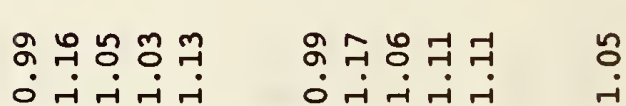

न

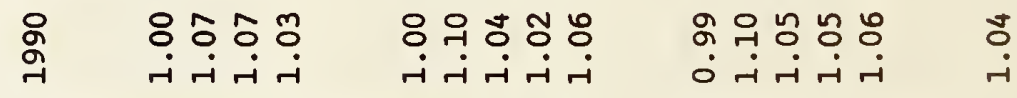

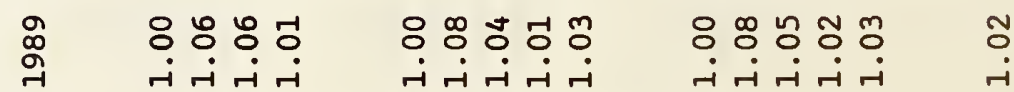

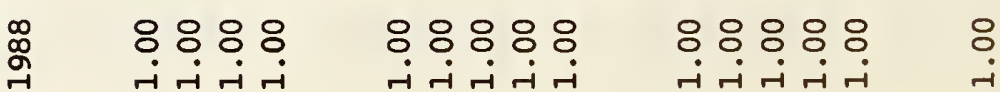

宝国

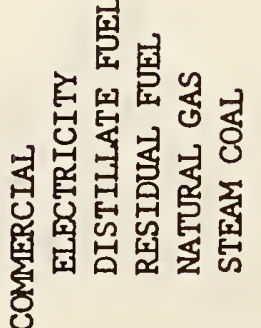




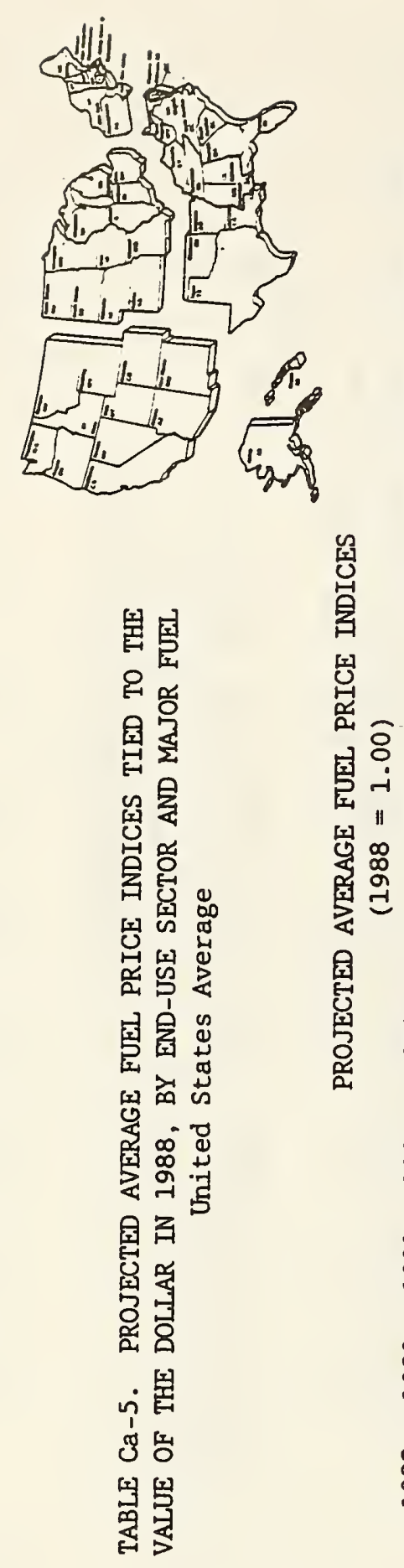

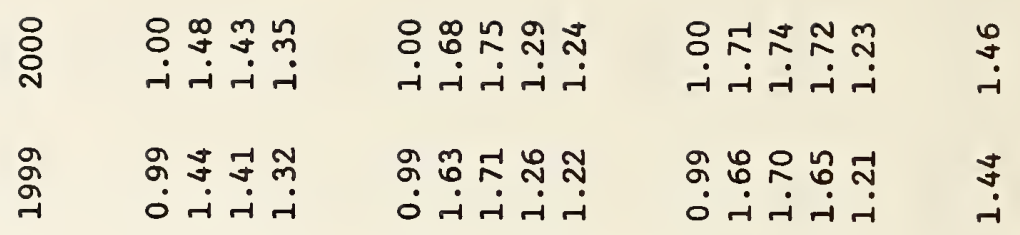

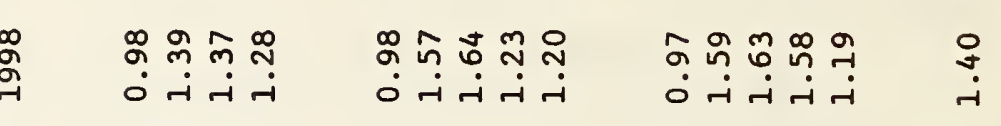

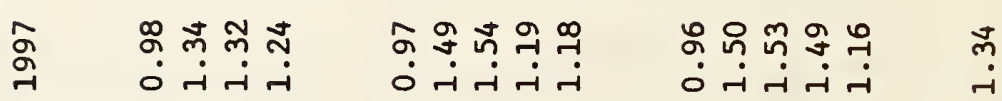

吕 कू̆

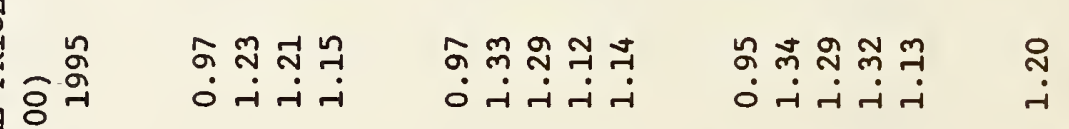

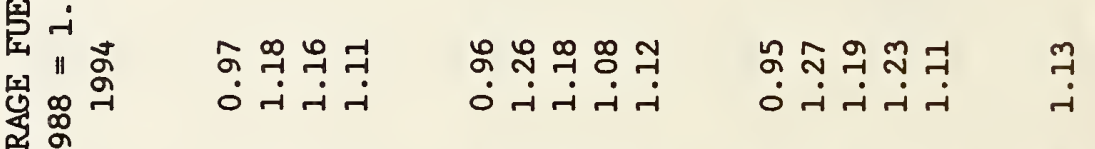

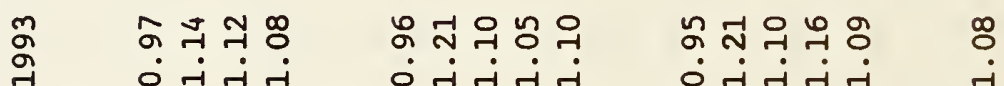

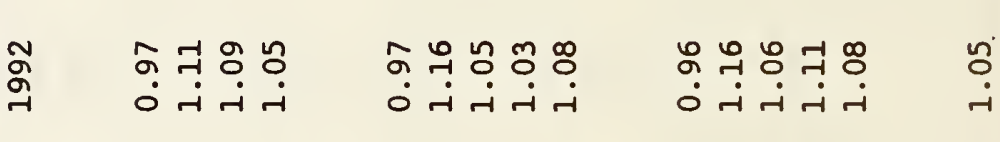

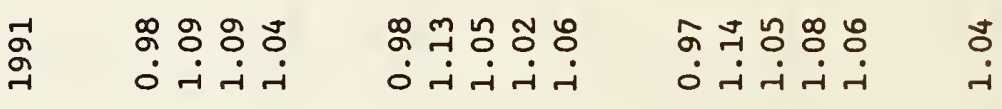

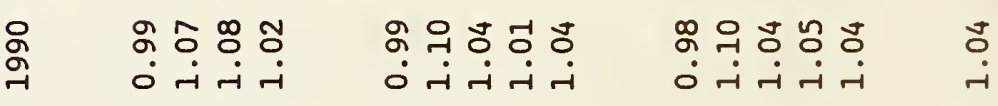

ळ

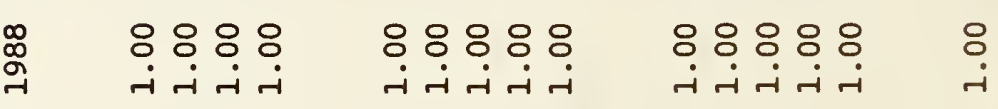

量 今

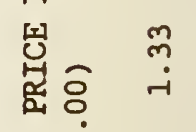

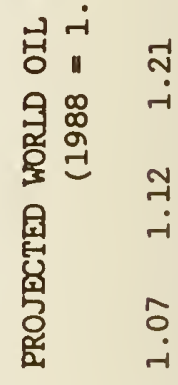

ํ.

$\stackrel{\circ}{\circ}$

$\stackrel{n}{\circ}$

$\stackrel{8}{\circ}$
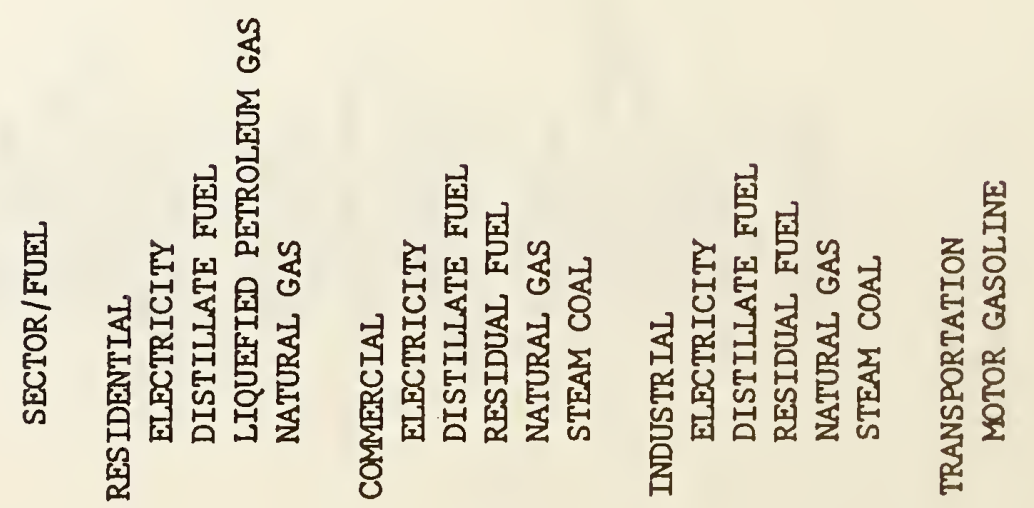

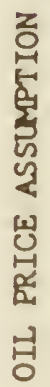




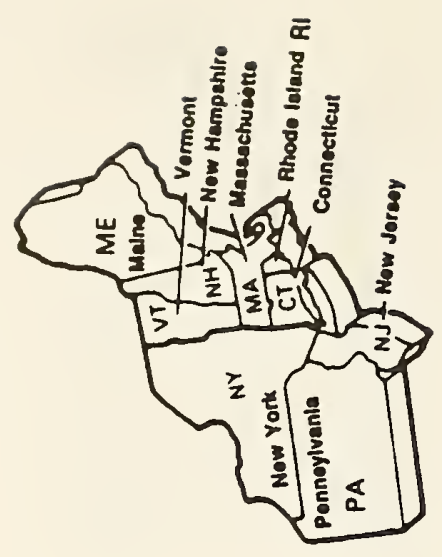

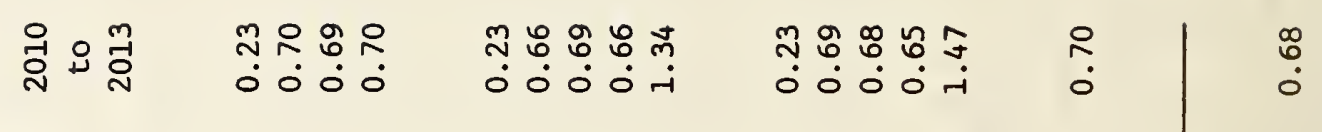

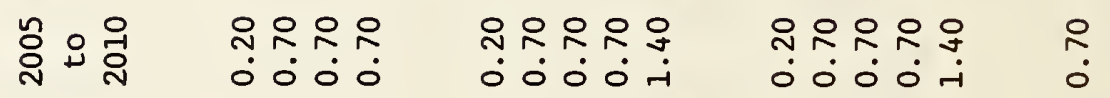

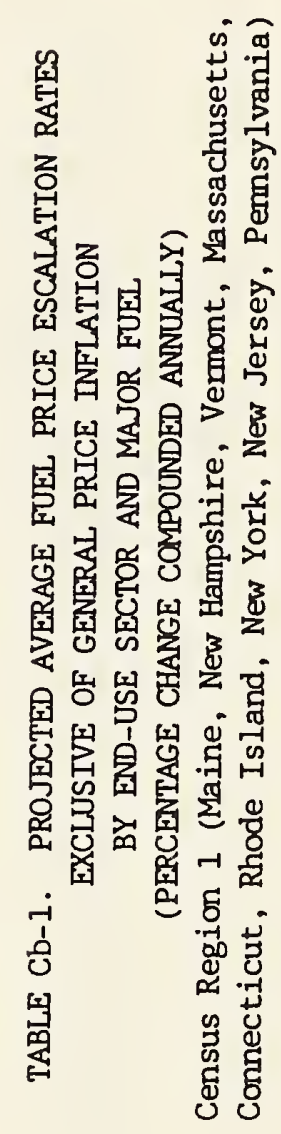

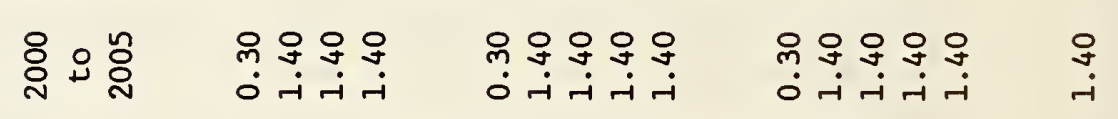

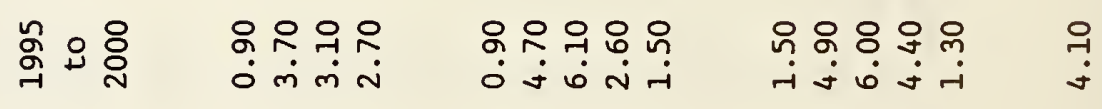

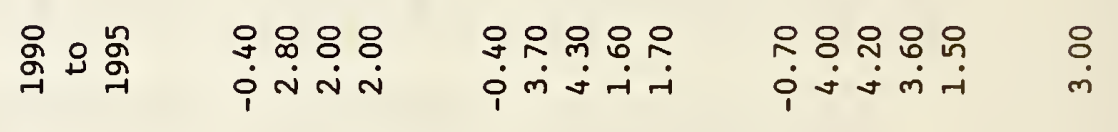

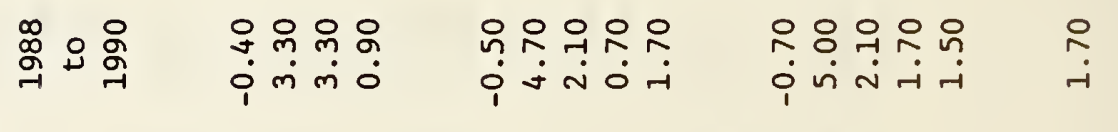
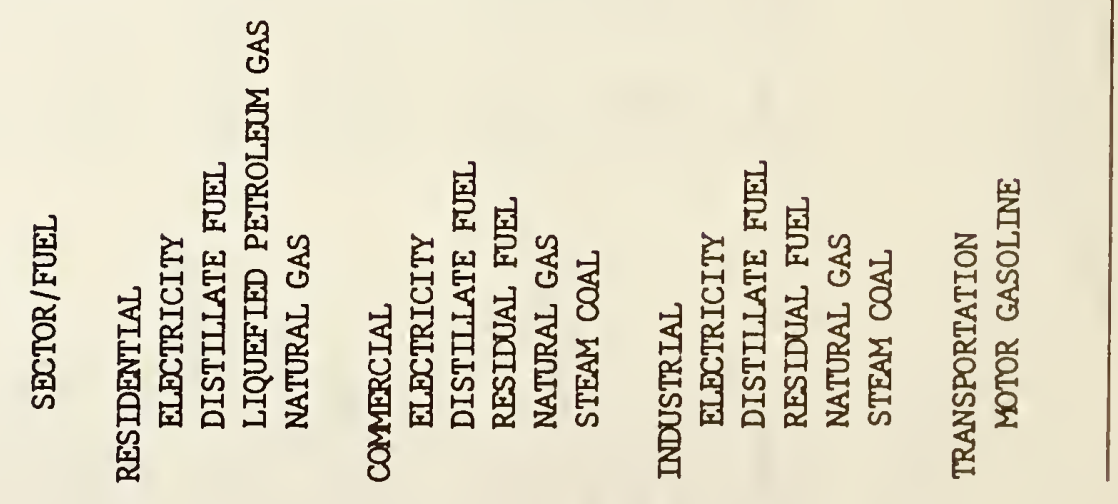

$\stackrel{0}{i}$

$\stackrel{\text { i }}{\text { i }}$

ํำ

$\stackrel{ }{\mathfrak{j}}$

$\stackrel{\circ}{\circ}$

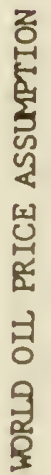




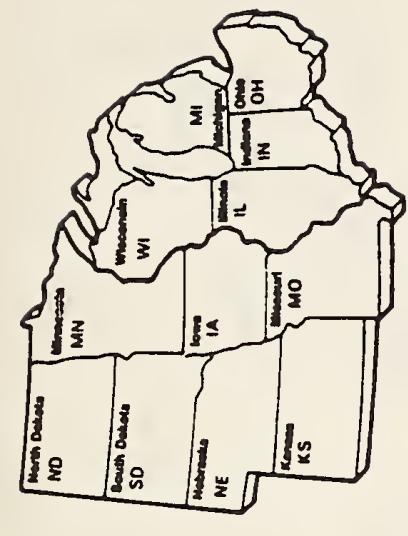

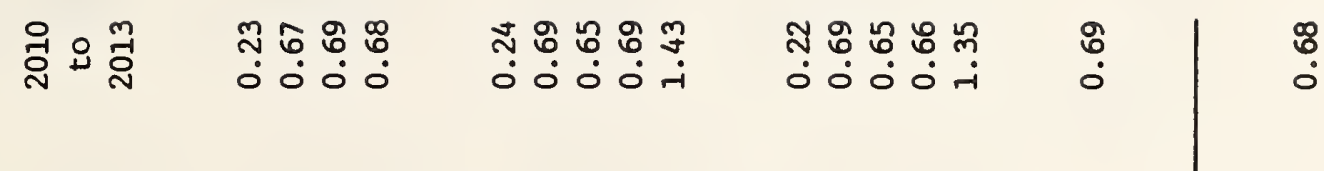

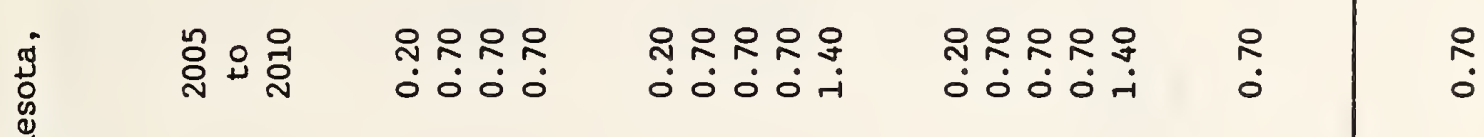

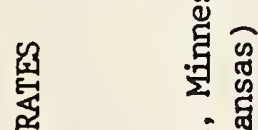

总

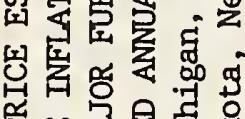

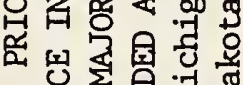

업 를

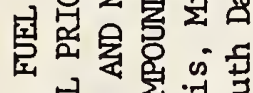

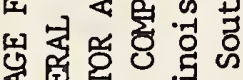

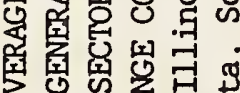

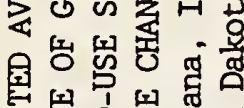

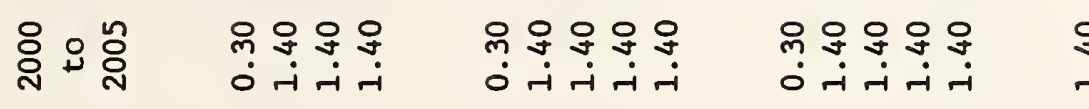

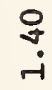

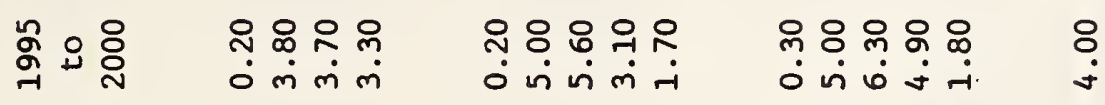

ถึ.

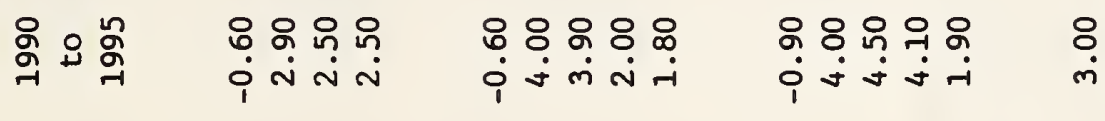

$\stackrel{?}{\dot{j}}$

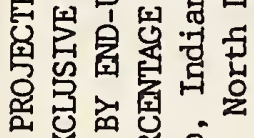

点

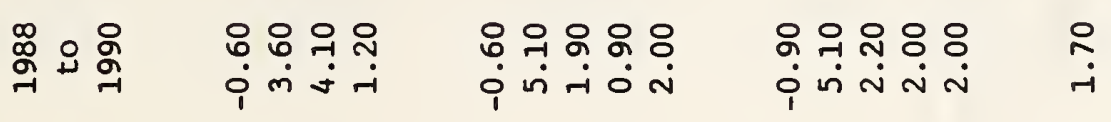

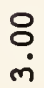

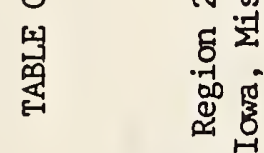

焉

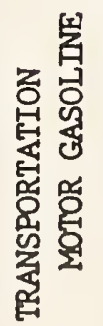

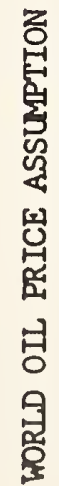




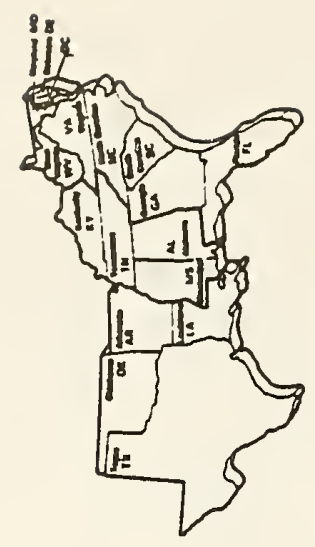

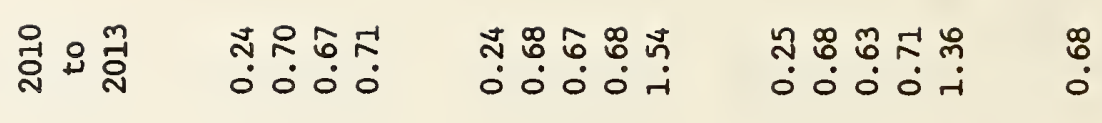

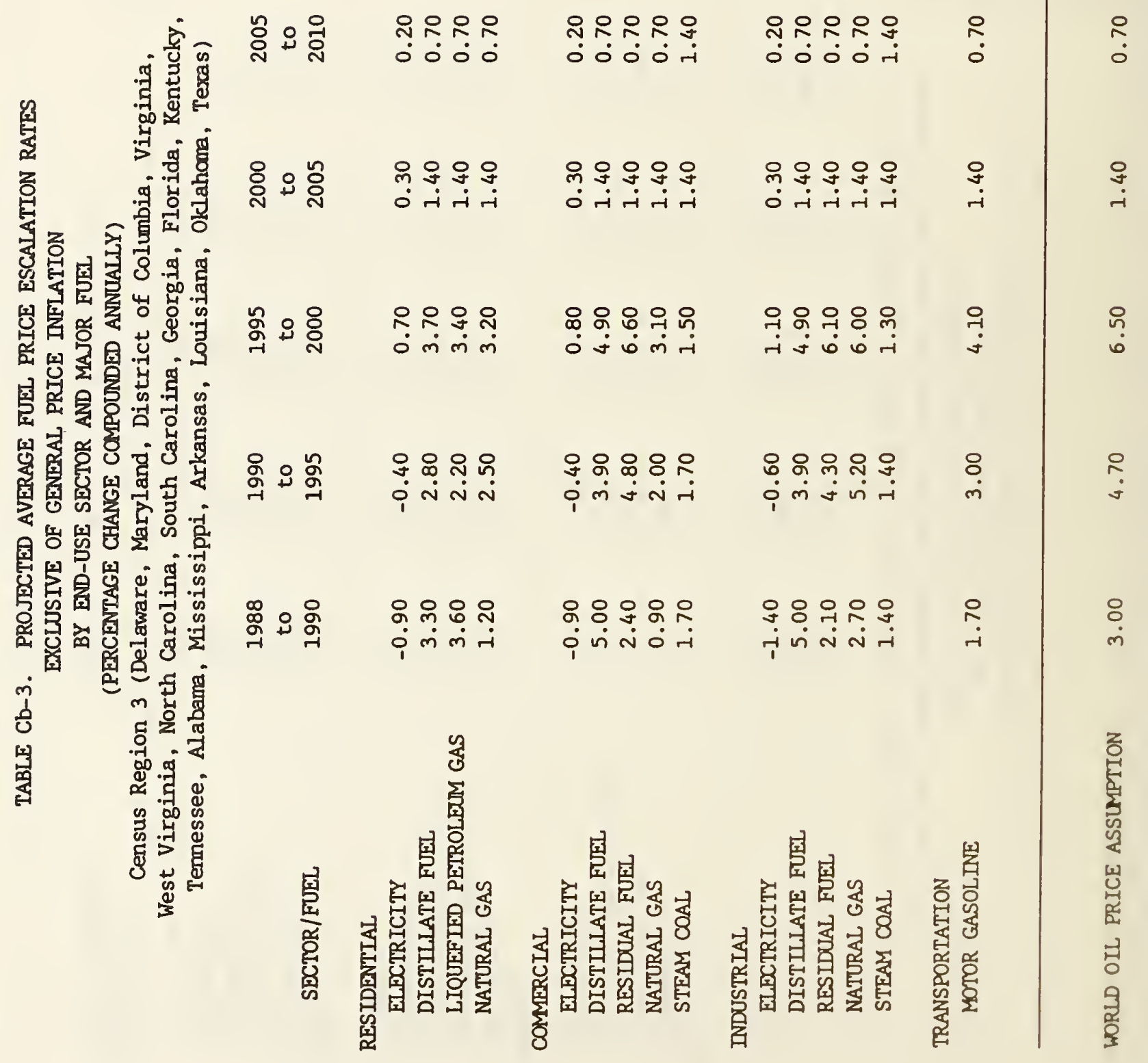




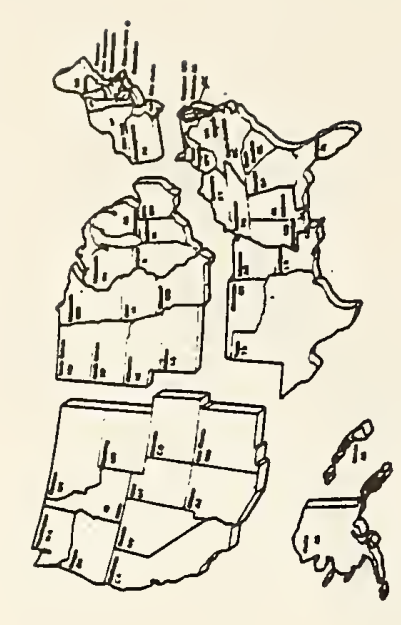

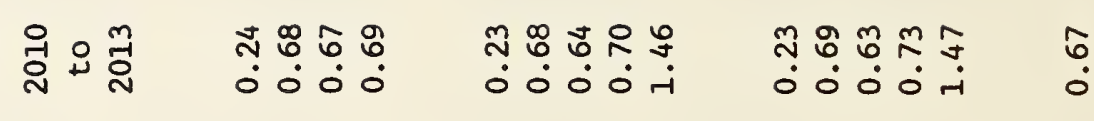

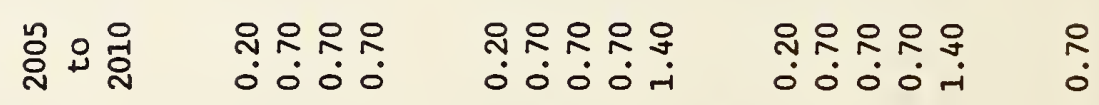

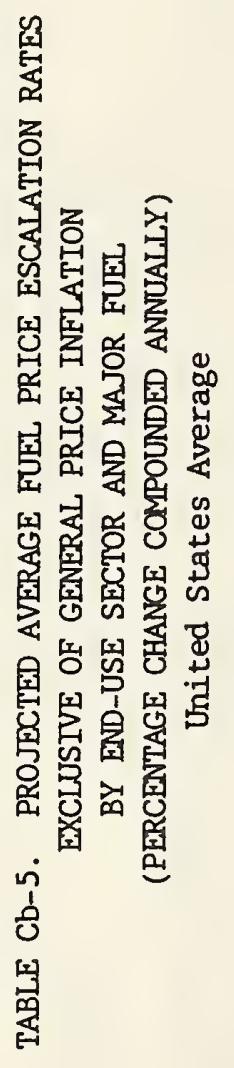

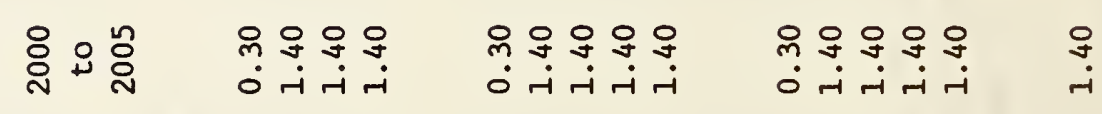

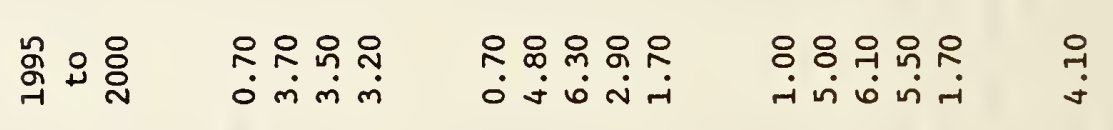

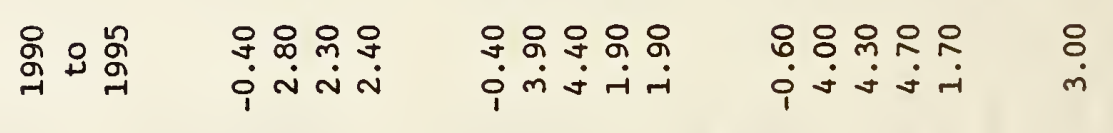

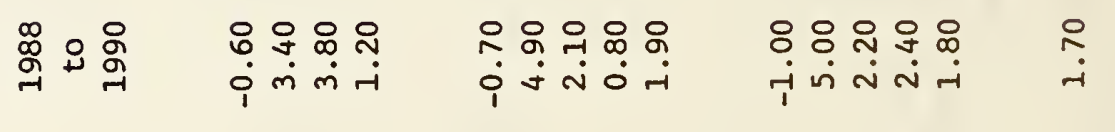
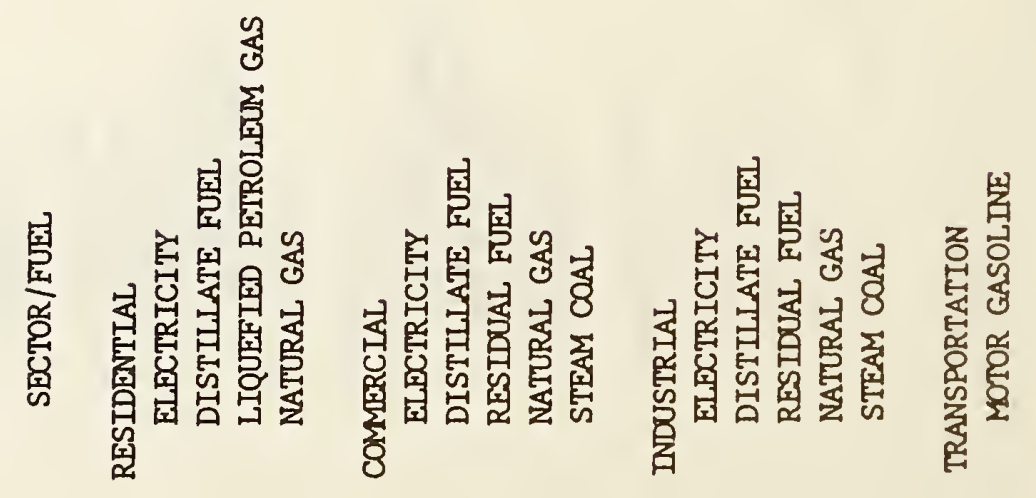

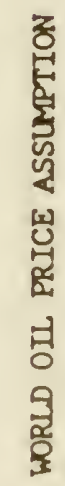


This section presents in tables S-1 through S-5 projected average fuel price indices for 4 fuels in the residential sector and 5 fuels in the commercial sector for each of the years from 1988 through 2013. They update tables originally published in the report, Comprehensive Guide for Least-Cost Energy Decisions (NBS SP 709).

As a convenience for the user, the indices include the effect of 4 alternative, hypothetical rates of general price inflation: 0, 5, 10, and 15 percent. Selection of these rates is intended in no way to suggest what actual rates might be. Use of the indices produce price estimates which are in current dollars, inclusive of general price inflation. Current dollar prices are needed when discounting is performed with discount rates which include general price inflation. For the case of 0 percent inflation, the price indices in the $S$ series of tables are identical to those in the counterpart $\mathrm{Ca}$ table series. When there is no inflation, there is no difference between constant and current dollars.

The indices based on inflation rates of 5,10 , and 15 percent allow the analyst to perform evaluations based on the assumption of a positive rate of general price inflation that changes the purchasing power of the dollar. Performing evaluations in current dollars is sometimes preferred for private investment decisions for three reasons: (1) it facilitates the treatment of taxes, (2) it allows the use of a market rate of interest for the discount rate which tends to be more readily understood by others, and ( 3 ) it explicitly treats inflation which also tends to be more readily understood by a lay audience than the constant dollar approach. But the current and constant dollar approaches give the same results if performed correctly. Analysis of private sector investments may be performed in either constant or current dollars. (Life-cycle cost analysis of Federal energy conservation investments should be conducted in constant dollars, using a real discount rate, as provided in tables $\mathrm{Ca}-1$ through $\mathrm{Ca}-5$. )

The indices in tables S-1 through S-5 are derived from the indices reported in tables $\mathrm{Ca}-1$ through $\mathrm{Ca}-5$ by means of the following equation:

$$
I_{S}=I_{C} \times(1+g)^{N} \text {, }
$$

where $I_{S}=$ Index found in tables $S-1$ through $\mathrm{S}-5$;

$I_{C}=$ Index found in tables $\mathrm{Ca}-1$ through $\mathrm{Ca}-5$;

$\mathrm{g}=$ Annual rate of general price inflation in decimal form; and

$\mathrm{N}=$ Number of discount or compound interest periods, in this case the year of the index minus 1988 .

Example of How to Use the Indices:

Suppose you wish to estimate the present value of energy savings in year 2000 , and you expect an annual inflation rate of 5 percent per year. Taking natural 
gas for residential use in Maryland, estimate present value savings as follows: (1) multiply the 1988 price for residential natural gas in Maryland by the projected quantity of energy savings in the year 2000, to estimate savings in the year 2000 in 1988 prices, (2) go to table S-3, find the year 2000 index for residential natural gas at a 5 percent inflation rate (2.44), and multiply by the result from (1) to determine savings in the year 2000 in 2000 prices, and (3) discount the savings back to the present, using an SPW factor based on a discount rate that reflects a 5 percent inflation rate.

For further explanation of the use of these indices, see NBS Special Publication 709, appendix B, Part I.

Note: The data in the tables which follow are now reported by 4 Census regions. Prior to the 1988 edition, regional data were presented by 10 DoE regions. Figure B-1 presents a map showing the states corresponding to the 4 Census regions. The Census regions do not include American Samoa, Canal Zone, Guam, Puerto Rico, Trust Territory of the Pacific Islands, or the Virgin Islands. Analysts of Federal projects in these areas should use data which are "reasonable under the circumstances," and may refer to the U.S. average data for guidance. 


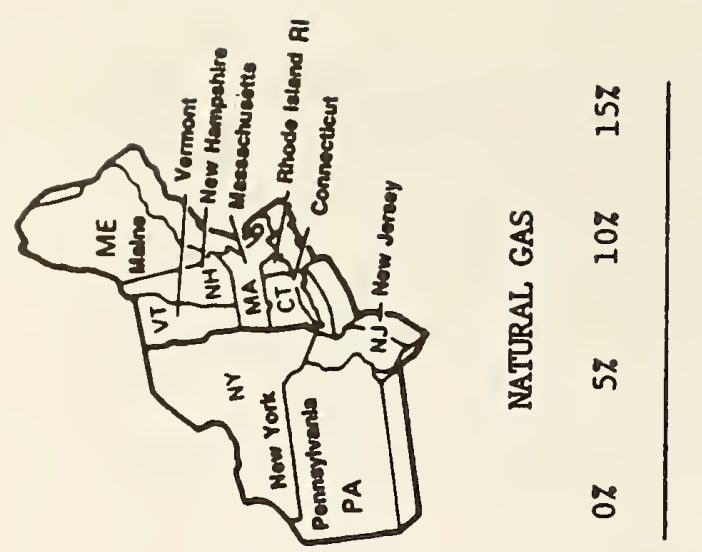

우

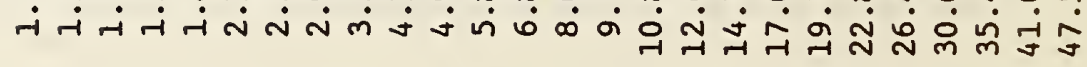

8규ำ -

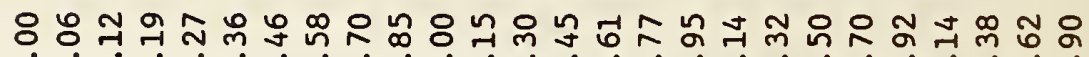

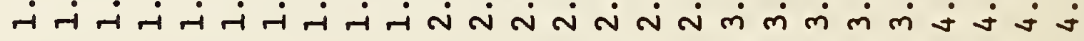

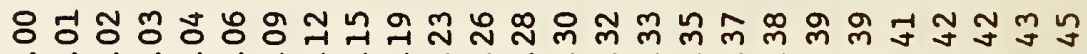

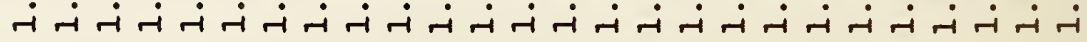

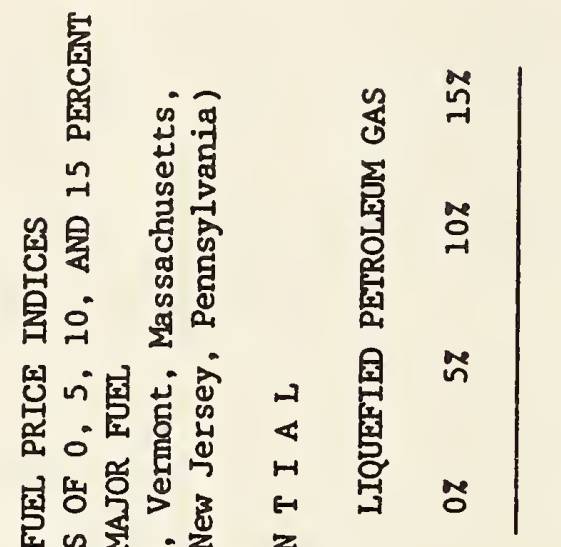

8 거

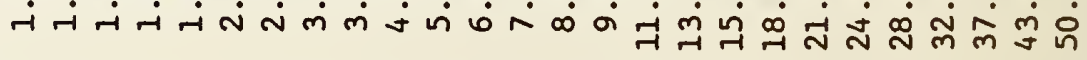

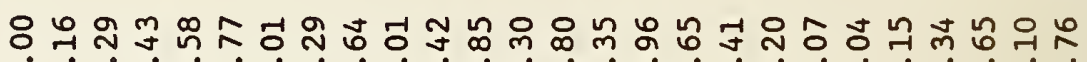
-i

각 ન્ન

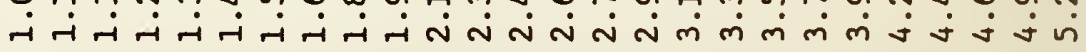

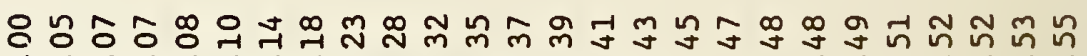

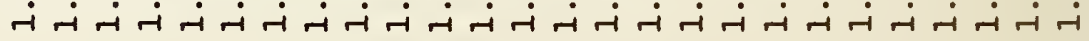

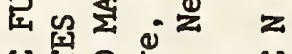

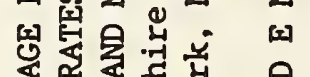

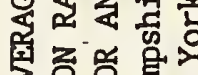

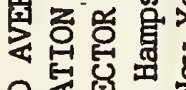

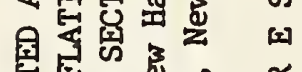

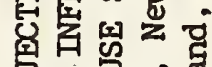

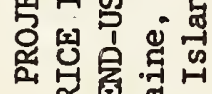

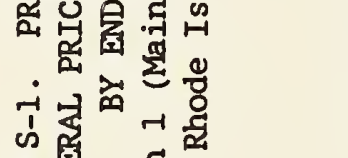

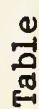

ह

范苛

ผี

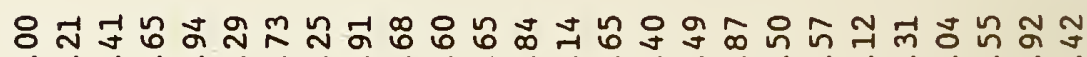

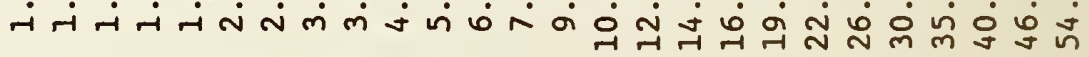

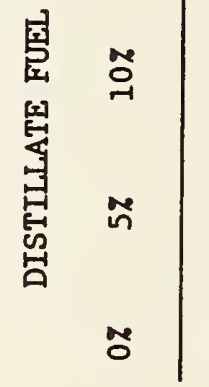

욱 워 웅

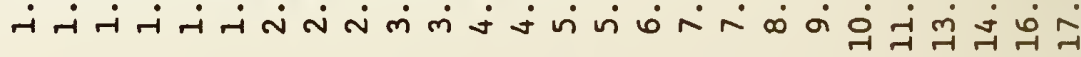

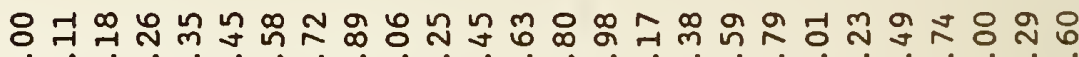

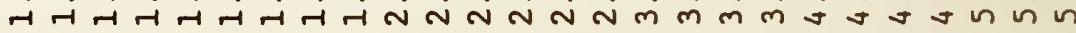

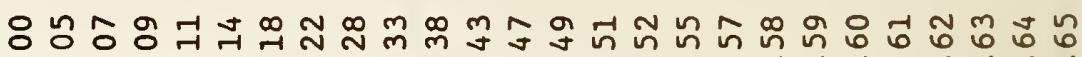

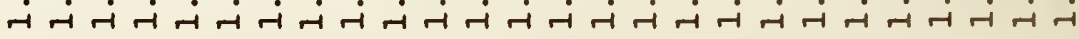

密 을

̋̊

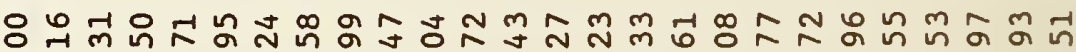
-i ન-iન નi

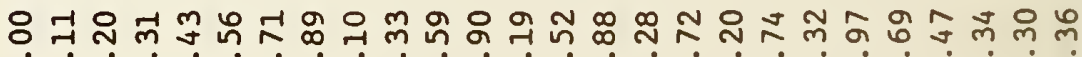

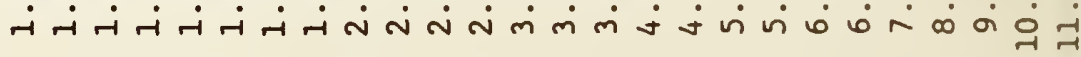

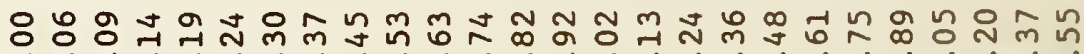

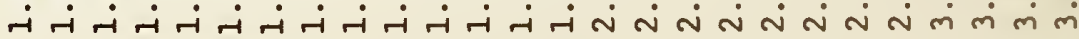

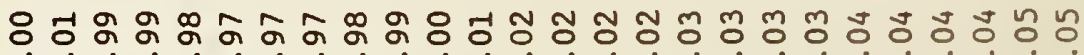

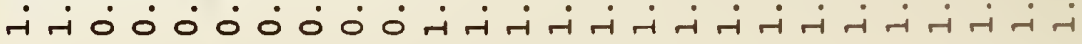

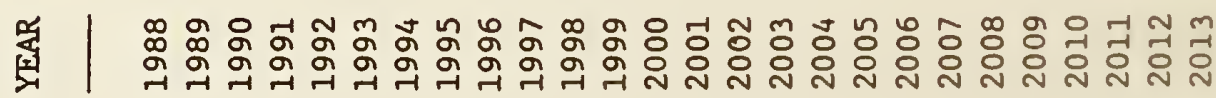




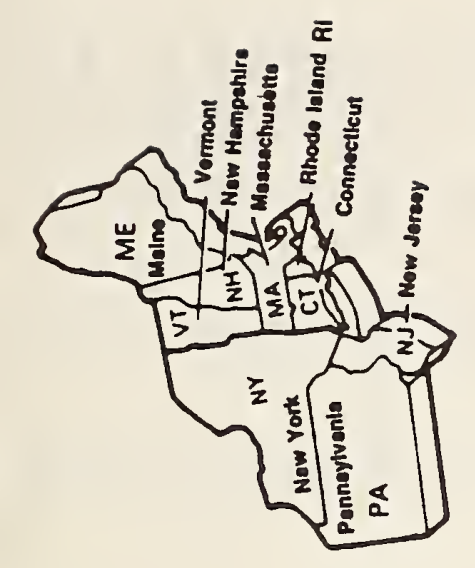

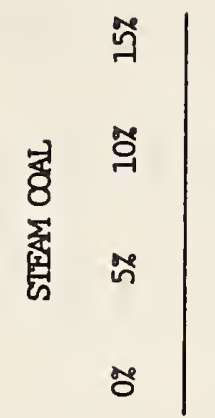

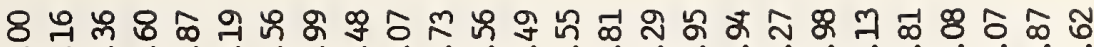

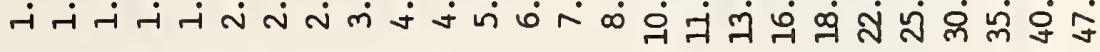

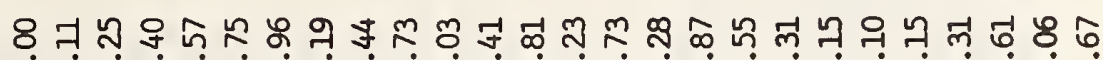
- न88 중요 웅

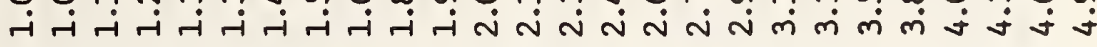

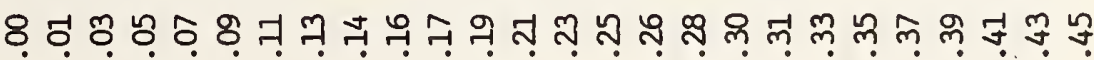
ن

ปี

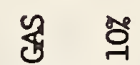

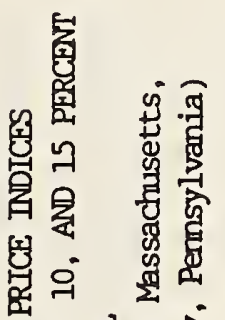

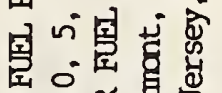

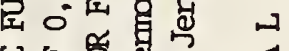

贸它务

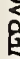

园击

罗悬窟

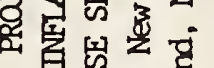

诸

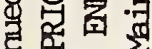

空

8

$-i$

के

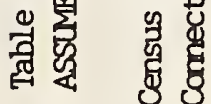

毁

ก้

$\not$

ที่

罢 总

ไี้

ถ้ำ

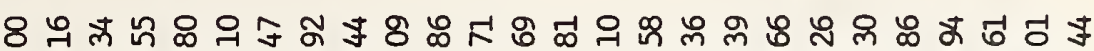
-

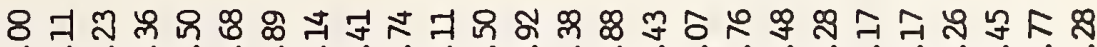
-

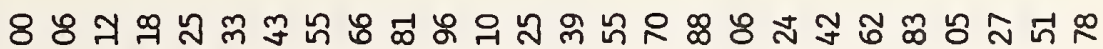

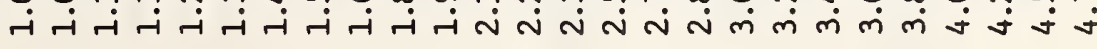

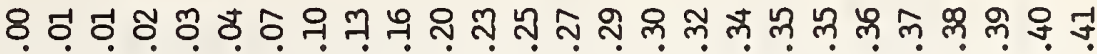

-

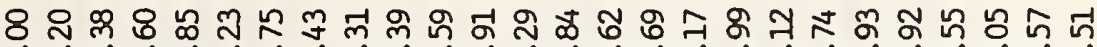

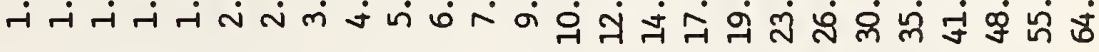

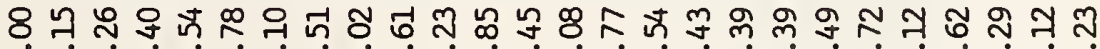

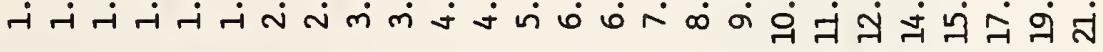

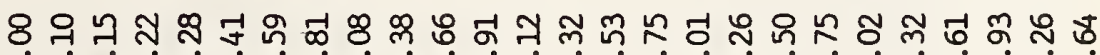

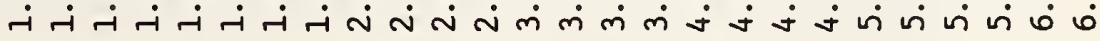

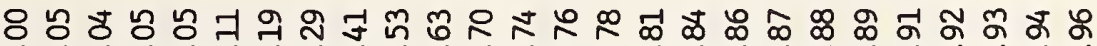

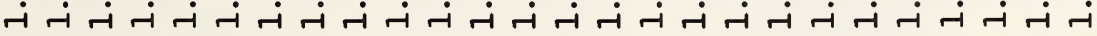

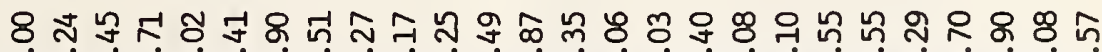
- ન-

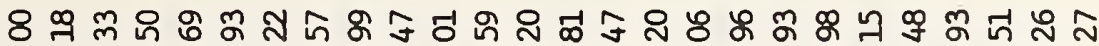
-

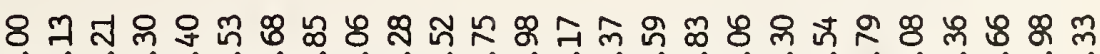
-

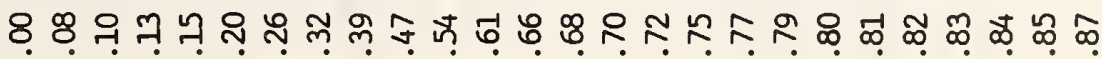

ที

穿

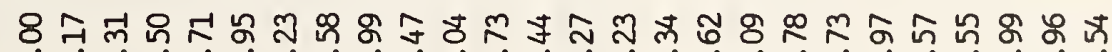

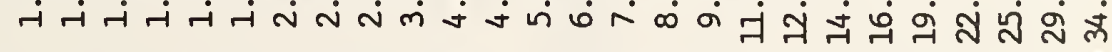

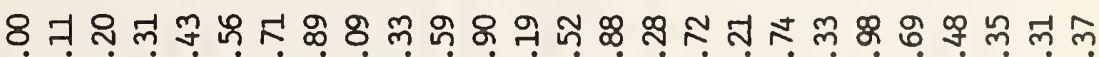

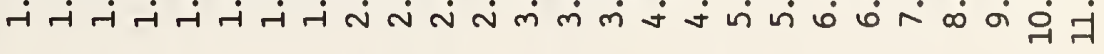

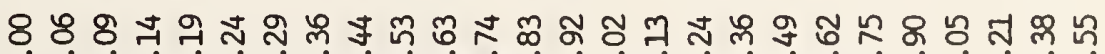

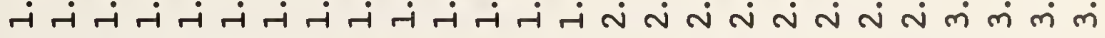

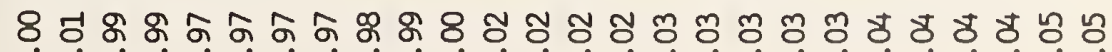
нi 


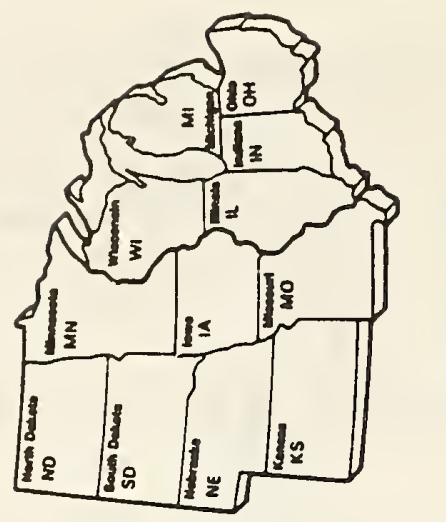

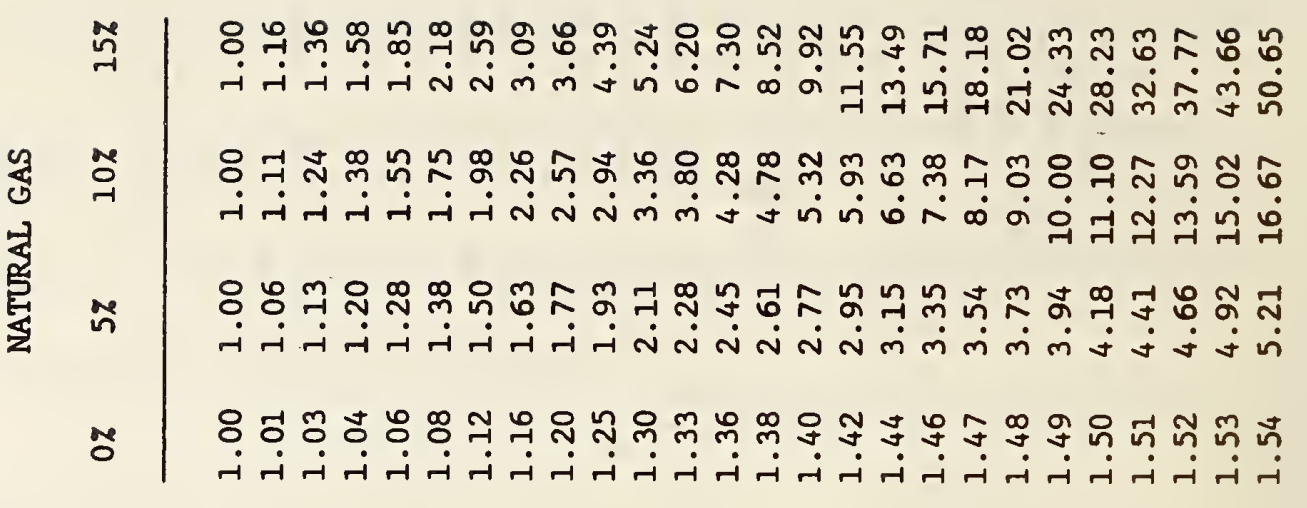

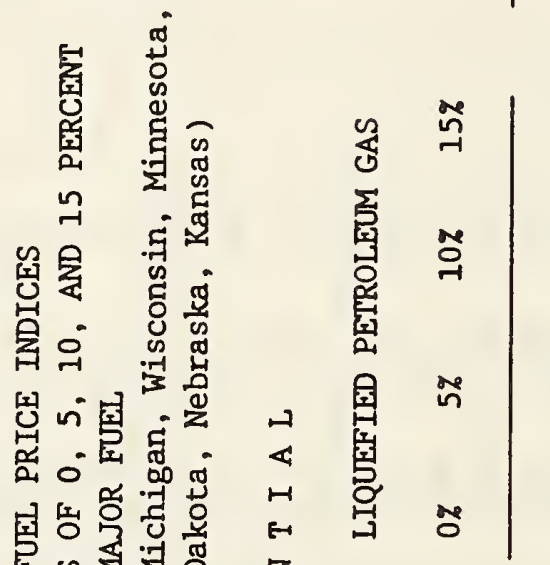

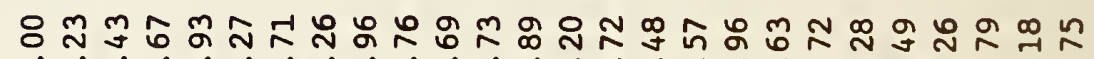

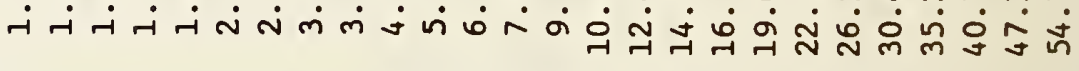

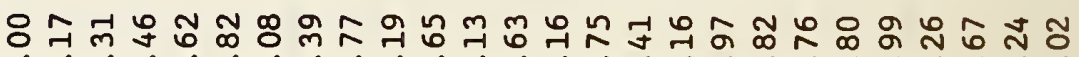
-

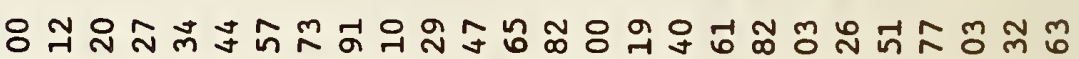

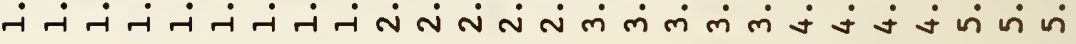

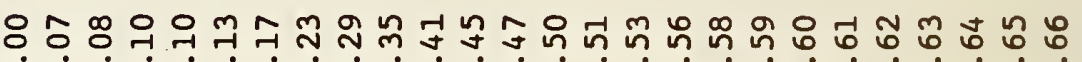

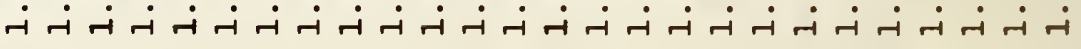

되

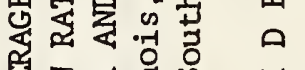

在实的 $H$

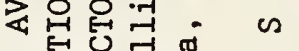

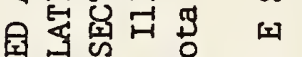

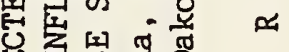

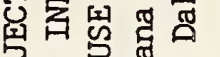

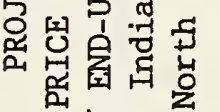

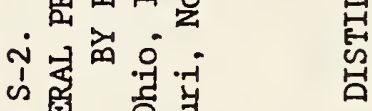

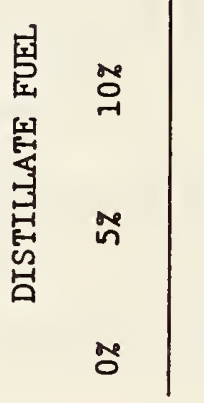

ปู

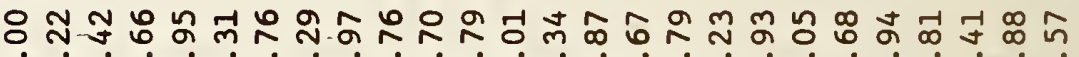

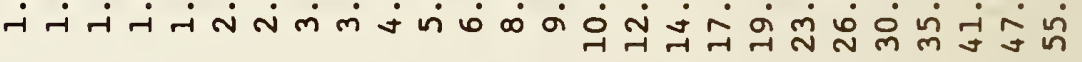

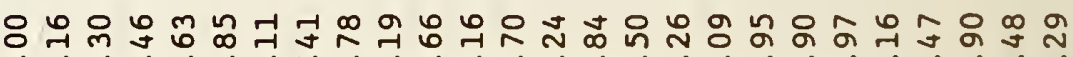

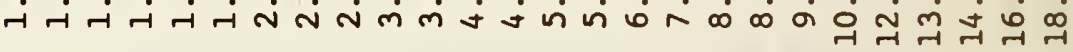

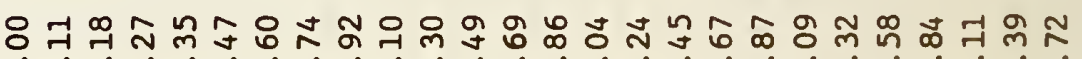
-

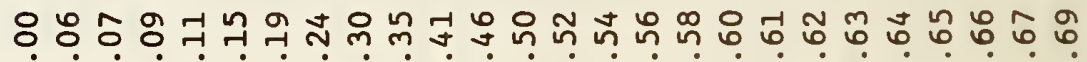

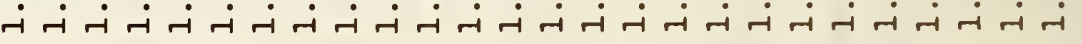

密

ڤั

윽

iี

re

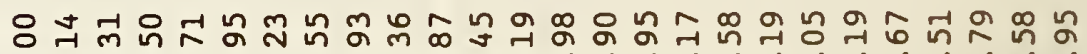

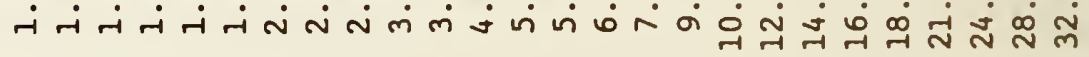

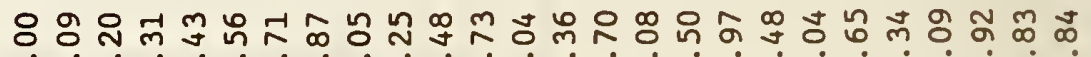

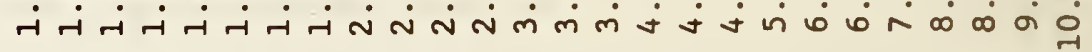

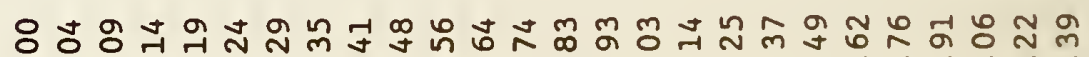

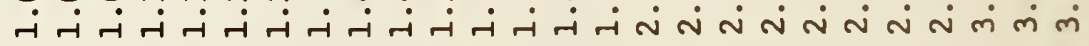

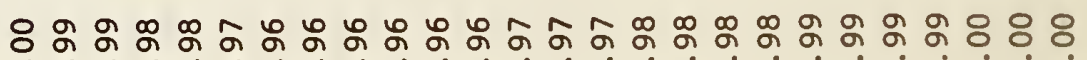
$\dot{i} \dot{0} \dot{0} \dot{0} \dot{0} \dot{0} \dot{0} \dot{0} \dot{0} \dot{0} \dot{0} \dot{0} \dot{0} 0 \dot{0} 0 \dot{0} \dot{0} \dot{0} \dot{0} \dot{0} \dot{\mu} \dot{\mu}$

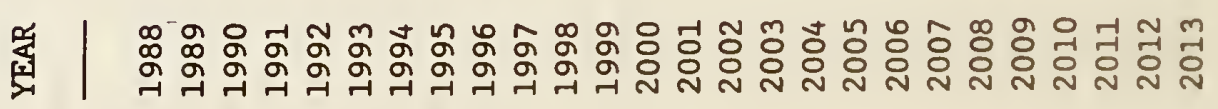




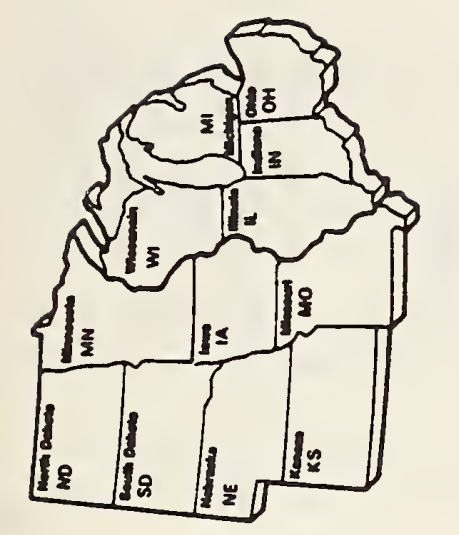

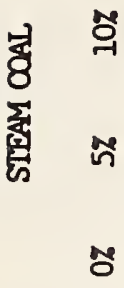

มี

ตั

娄 气ै

๙̋

ที่

圆 을

ถู

๙

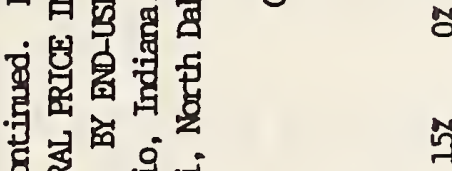

圆

高

ถ้

\&

ตี

珀

8 नิ ติ ન- ન- નi N

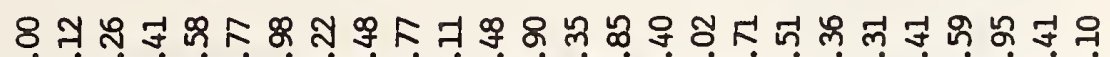

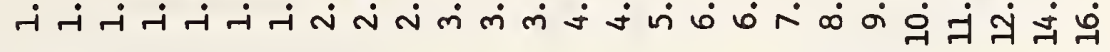
8 ㄴำ ส

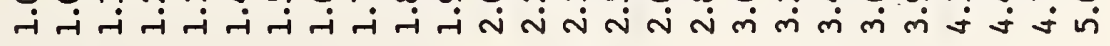

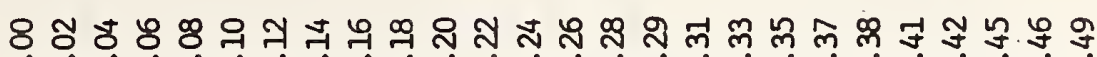
म

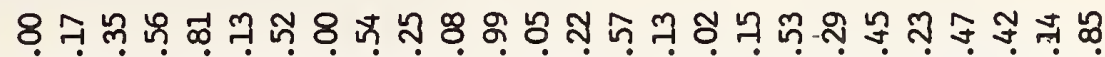

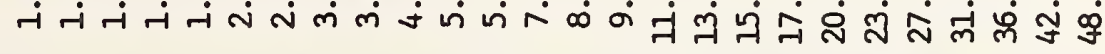
8 ๆ ๆ

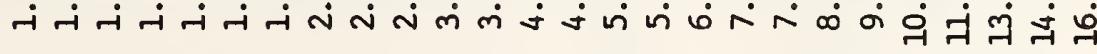

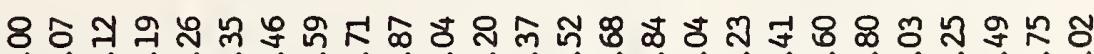

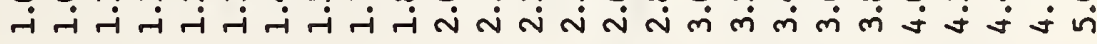

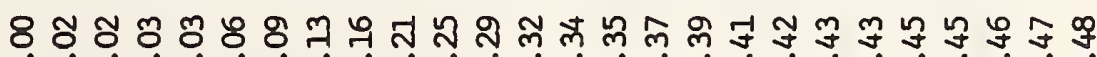

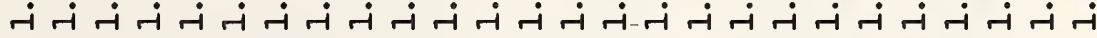

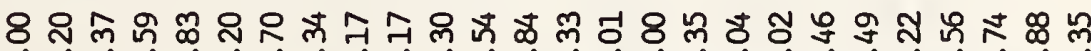

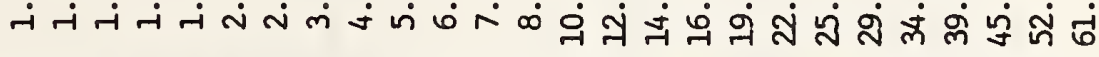

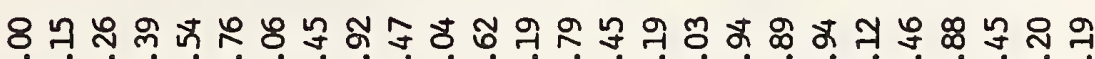

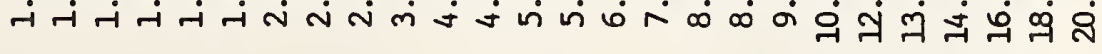

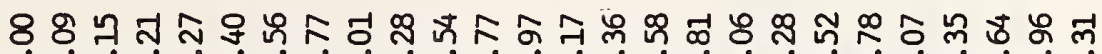
-

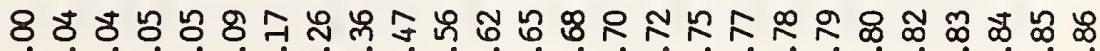
નં

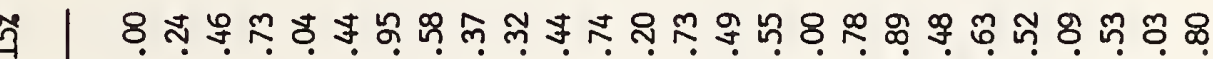
ન- ન-

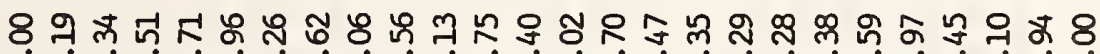
-

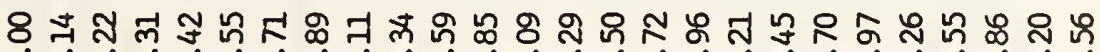

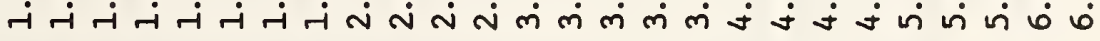

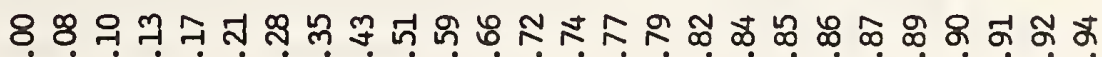

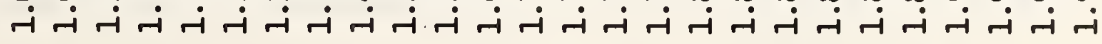

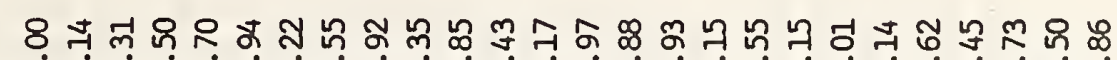

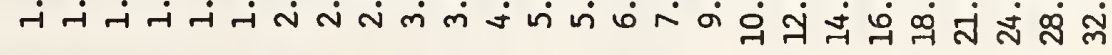

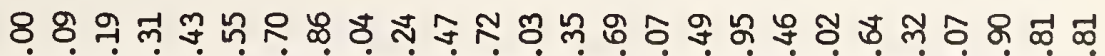

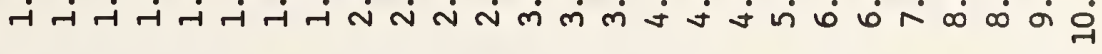

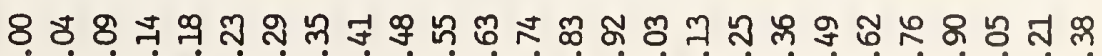

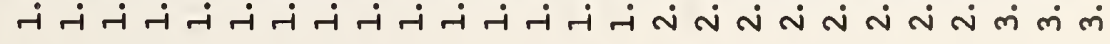

8 \& \&

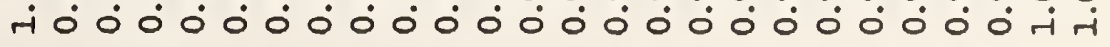

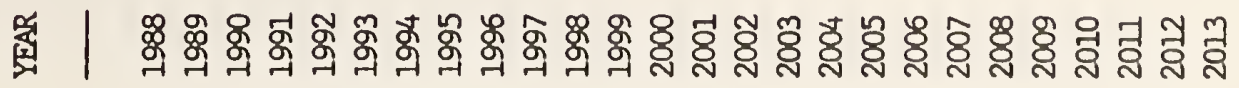



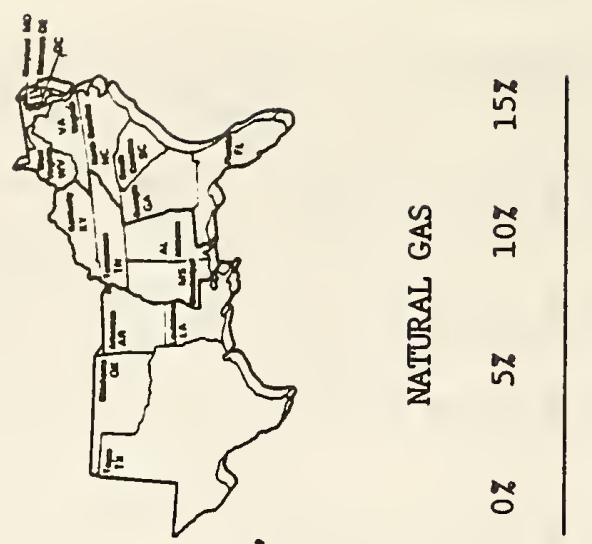

욱 ๆ

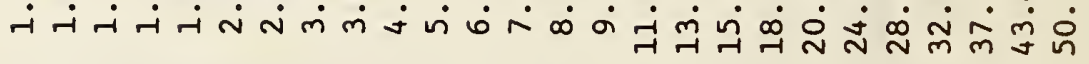

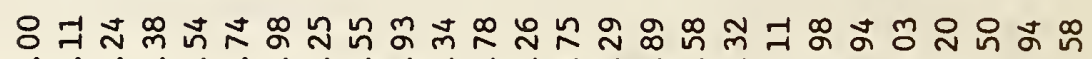

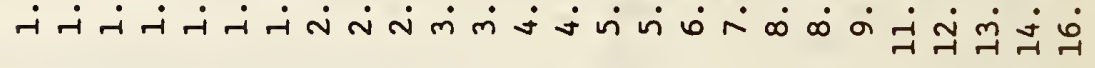

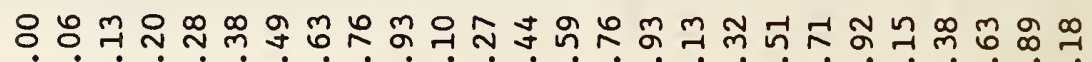

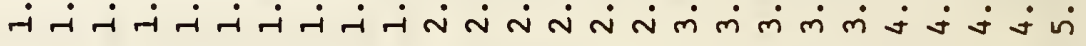

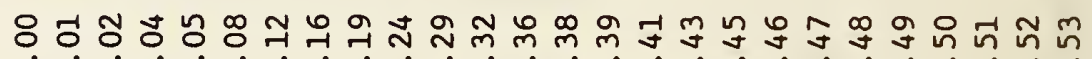

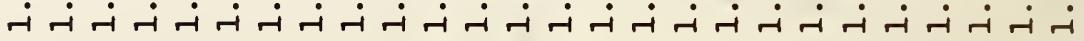

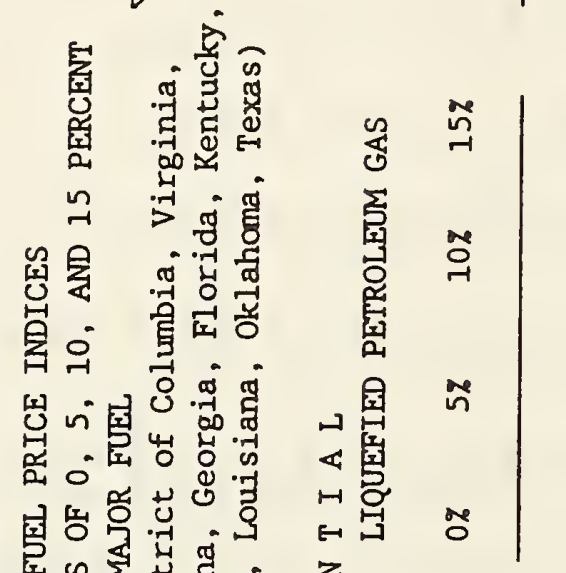

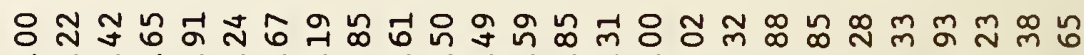

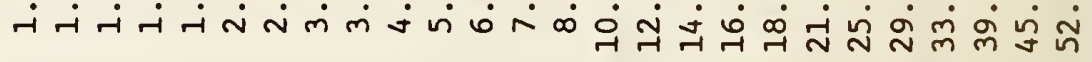

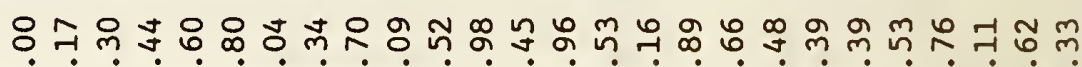

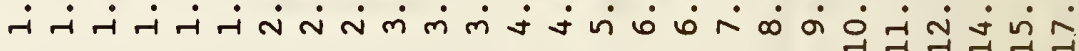

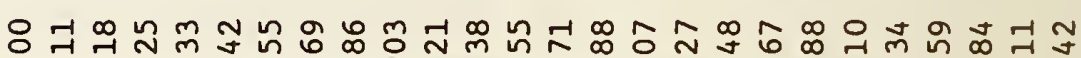

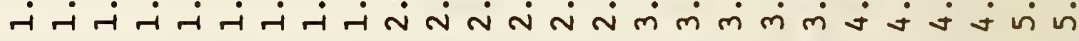

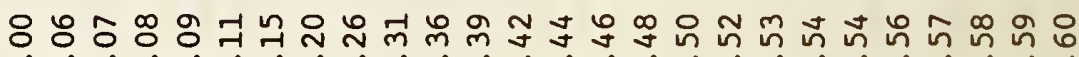

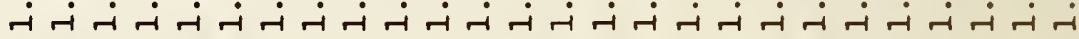

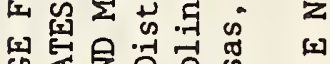

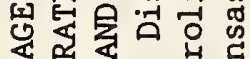

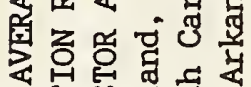

ค

$\mapsto$

es

ติ

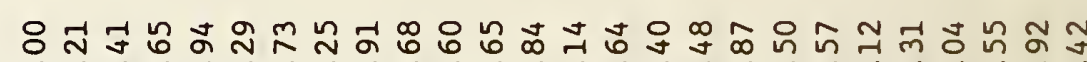

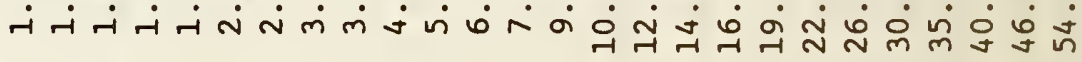

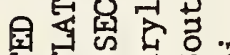

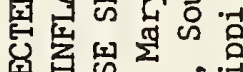

(⿻)

幽国

움

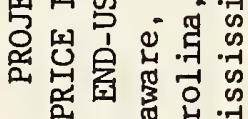

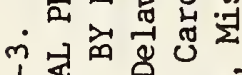

告

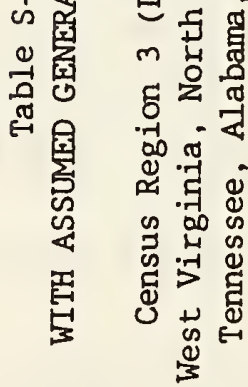

疽

ที

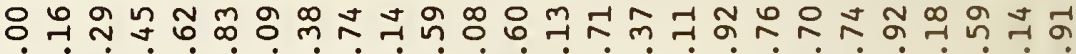
-i नંनં

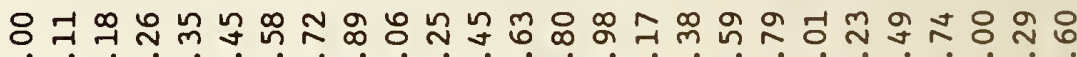

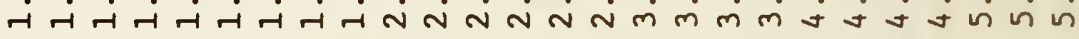

oe

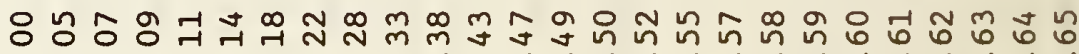

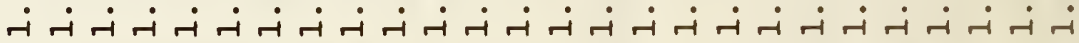

䆖

ติ้

엉임의

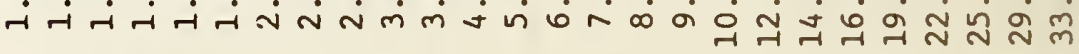

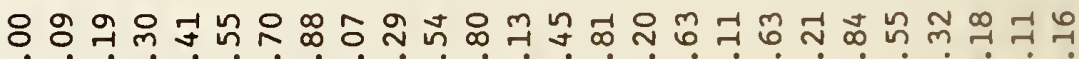

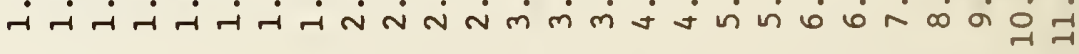

ㅇํㅇำ

ñ

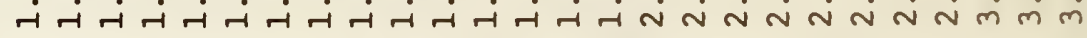

me

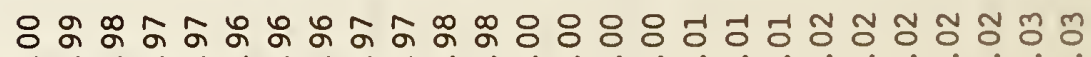

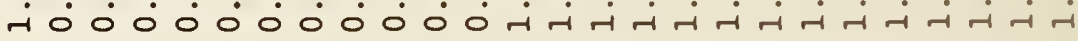

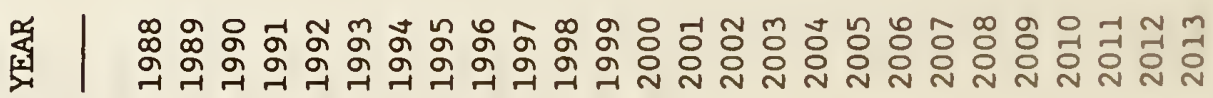



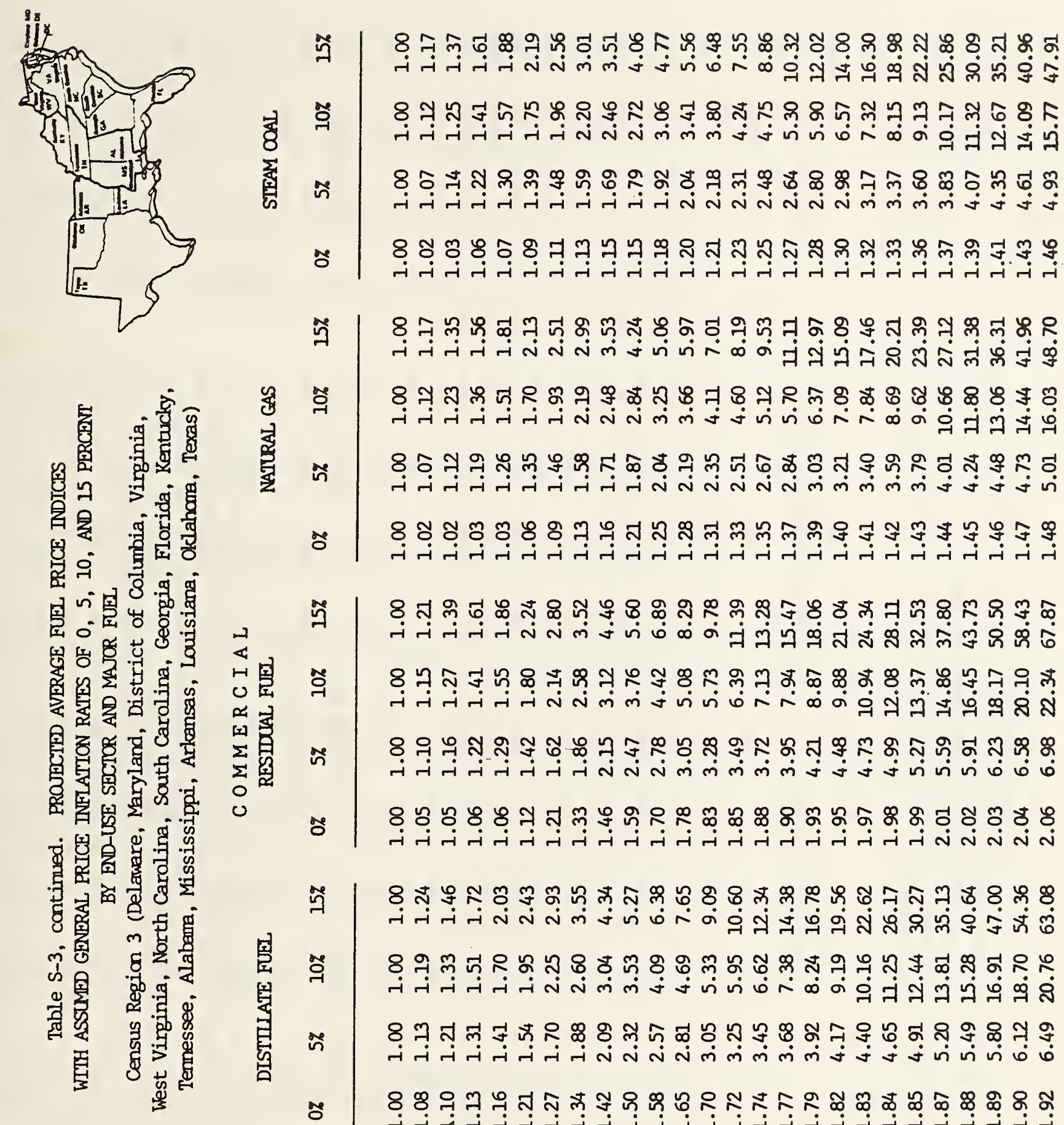

\section{قั}

8 ๆ ن

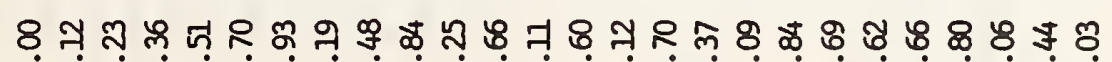
-

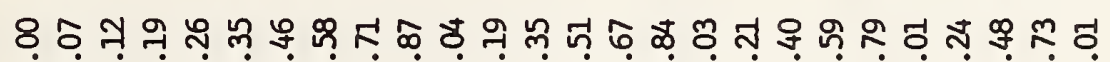
- नं न न न न न न

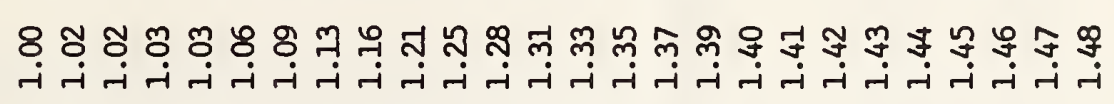

ติ

$\rightarrow$

4

$\rightarrow$

$\alpha \rightarrow$

[a]

$\Sigma$ 采

0

0

今̊

๓ึ

8 ส -

$\approx$

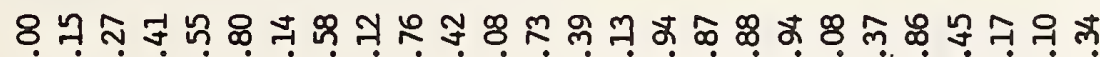
-

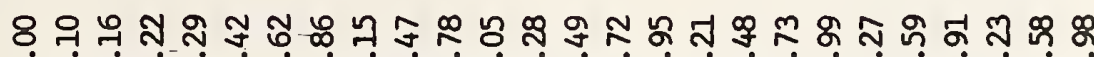

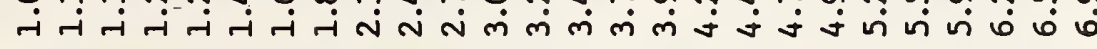

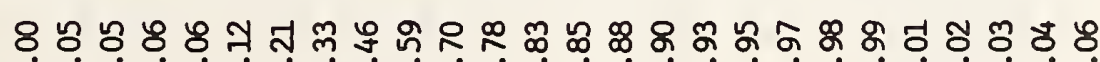
-i

ตี

䍚

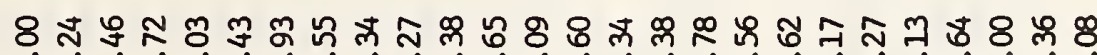

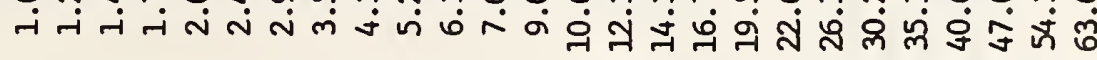

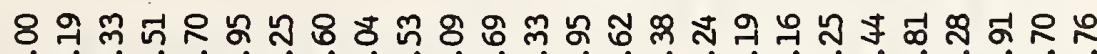

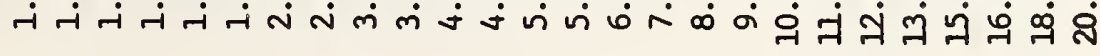

ถ้

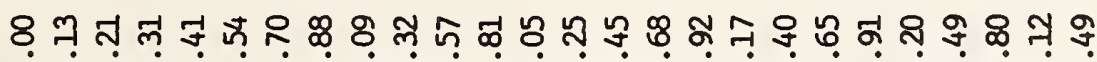

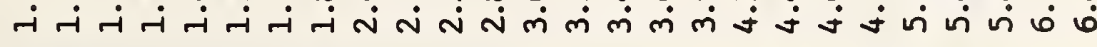

웅

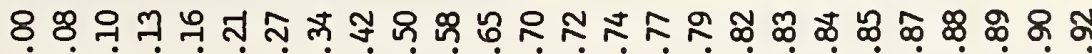

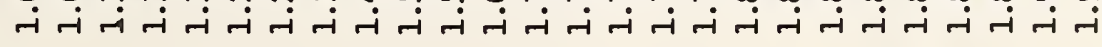

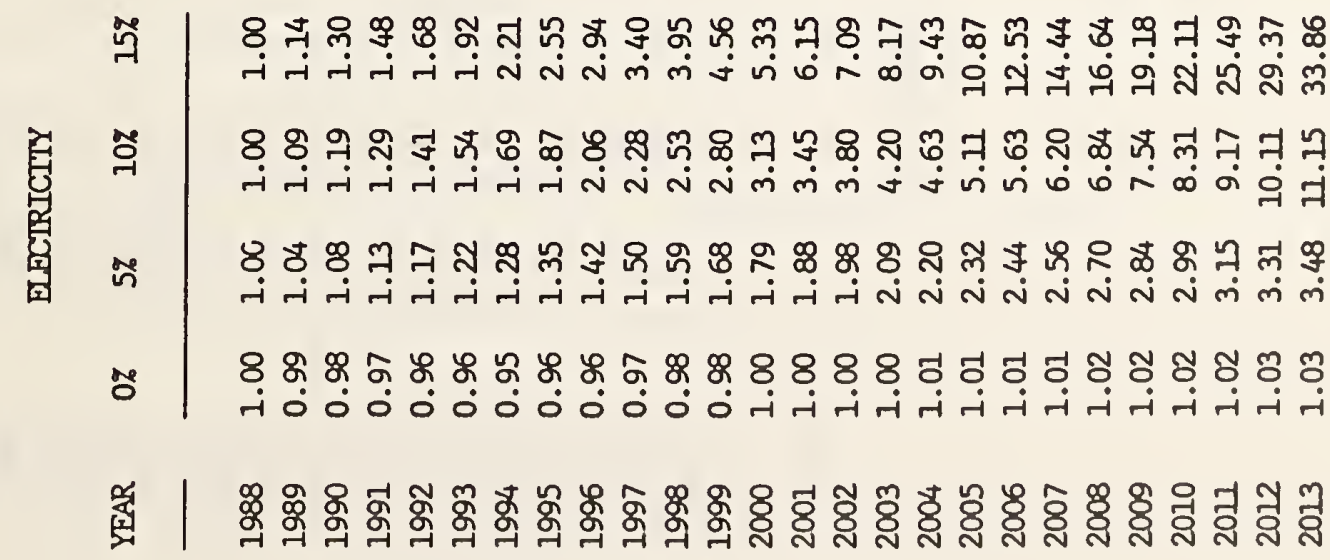



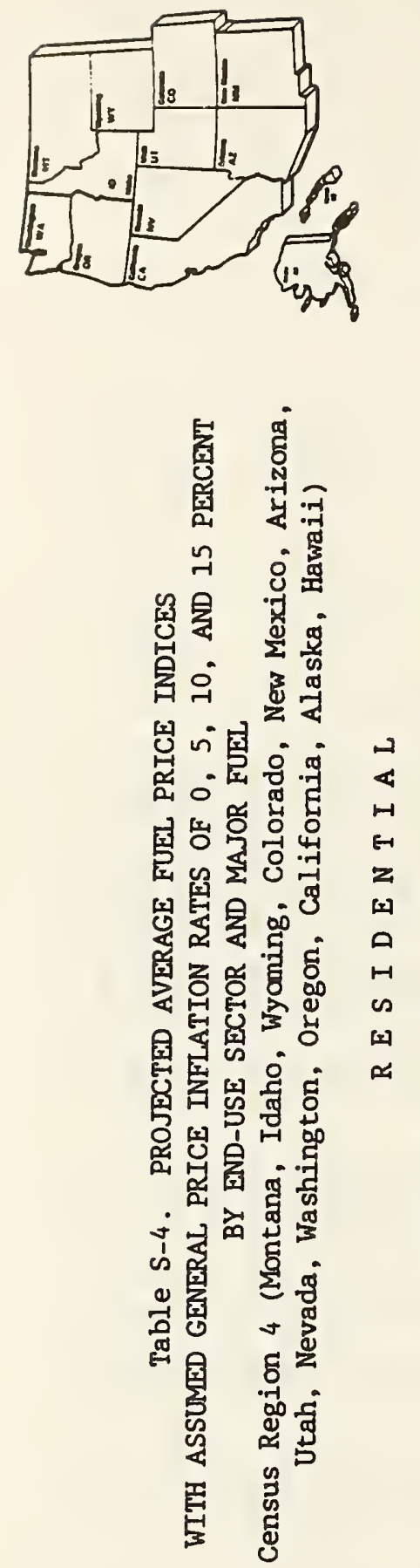

电 مै

ที

No

窇

Эั

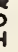

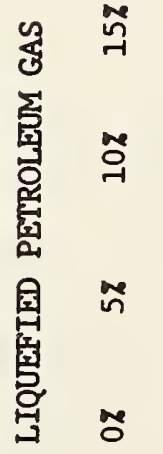

$\mid$

国是

气ี

穿 울

$\ddot{\circ}$

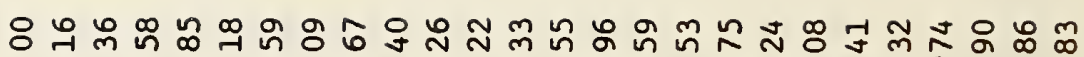

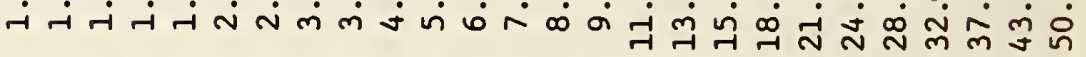

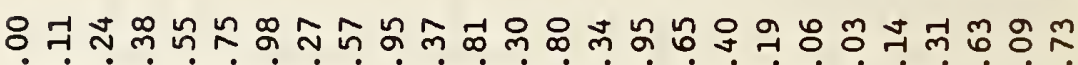

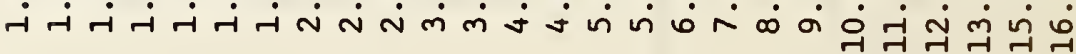

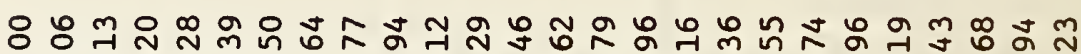

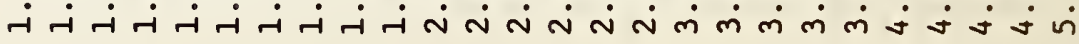

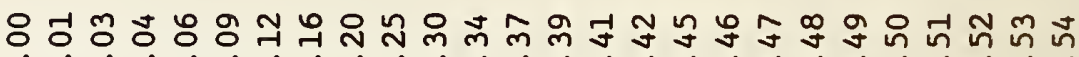

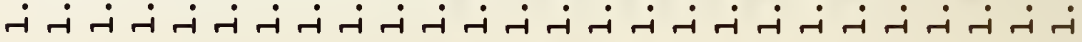

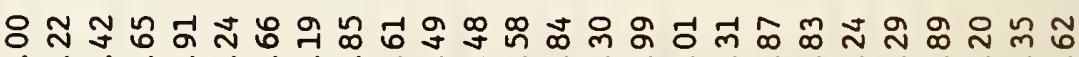

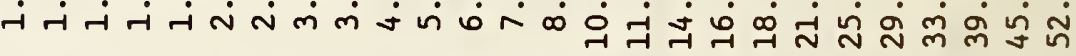

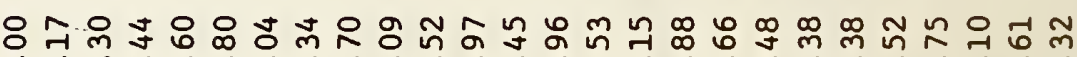

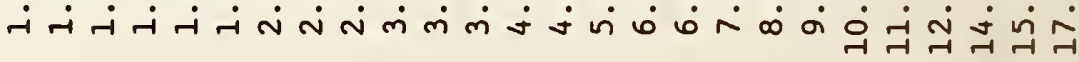

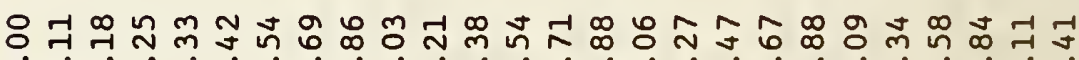

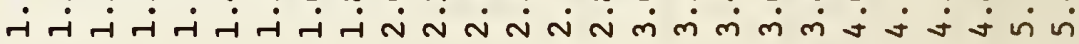

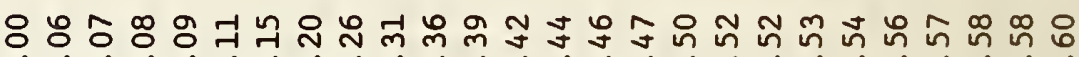

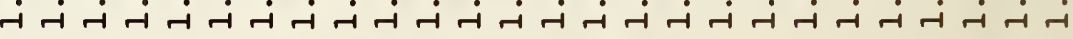

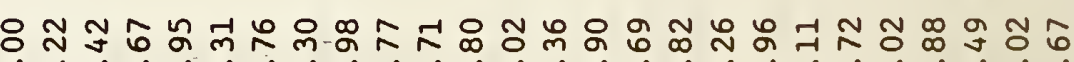

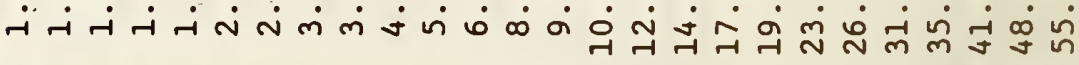

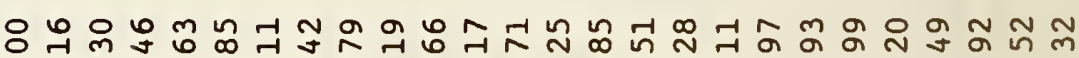

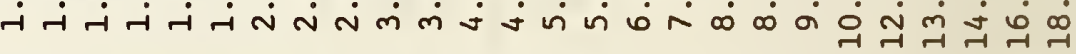

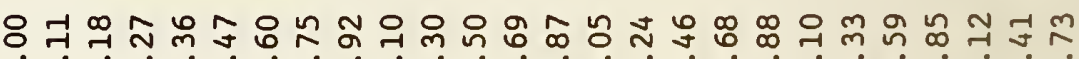

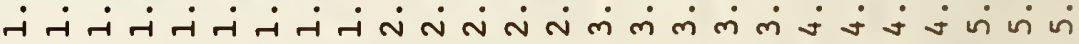

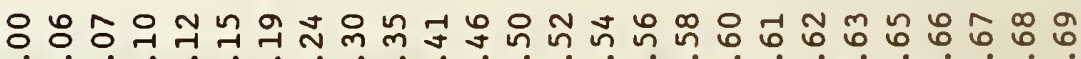

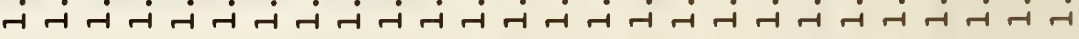

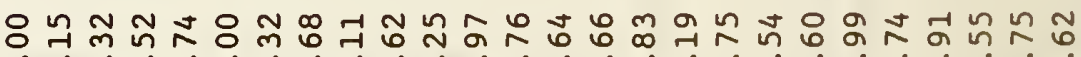

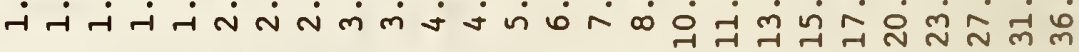

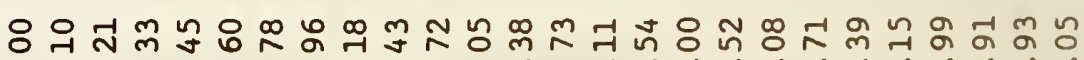

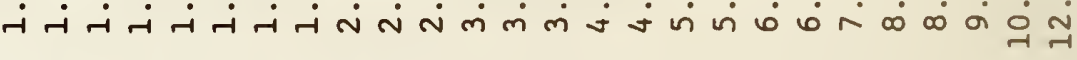

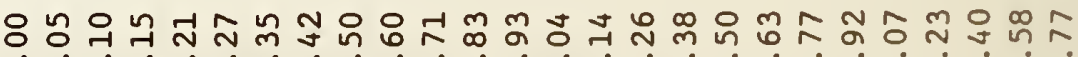

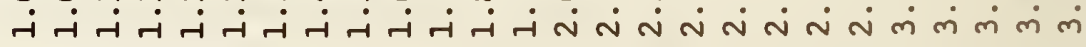

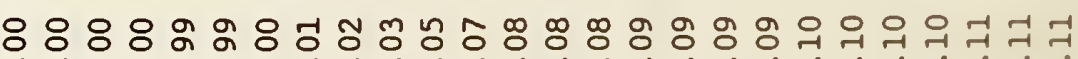

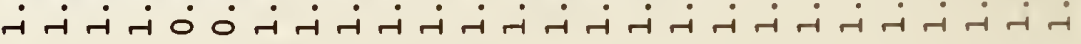

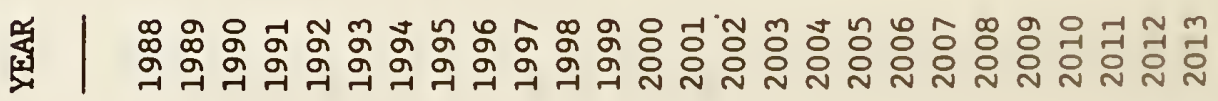




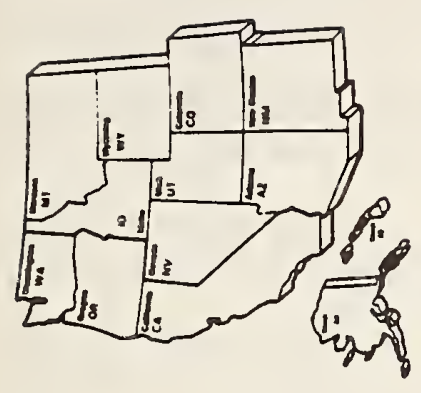

8 ஜ 8 8

ท้ 8 8 స -iન-i નi N

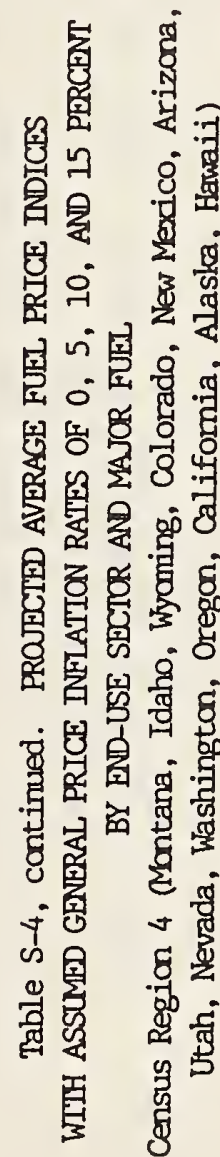

8 귀 ๆ -i નં નં નi 8 \& 7 ศ -i 8 бㅇㅇㅇㅛ

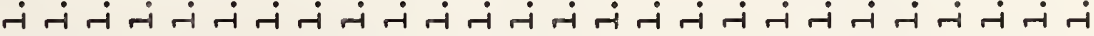

น้ำ

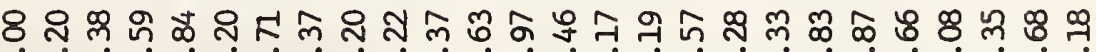
-i ન- -

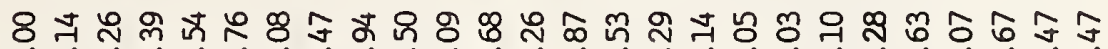
-i

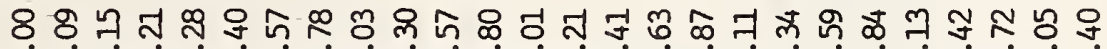

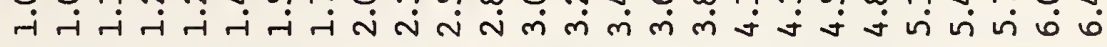

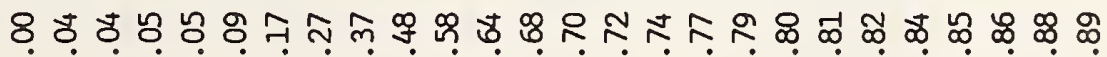

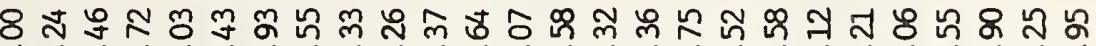
- ન નi 8 ㅇำ ન ન ન ન ન

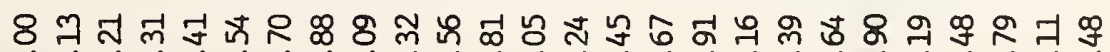

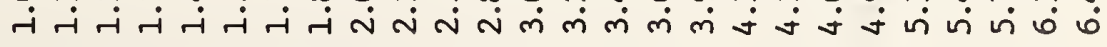
8 8으국ㅊำ

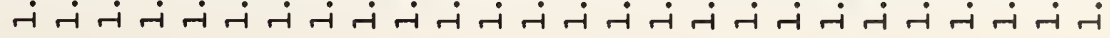

ต้

量 ํㅡㄹ

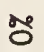

受

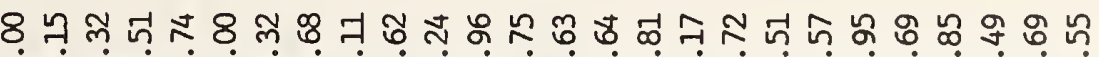

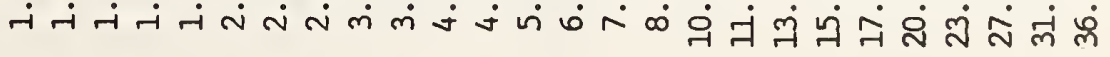

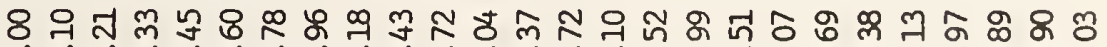
-i ન- ન ન

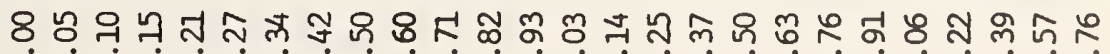

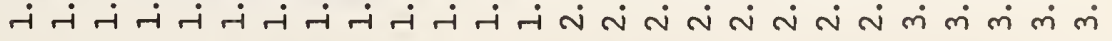

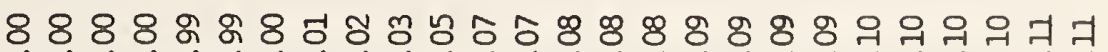

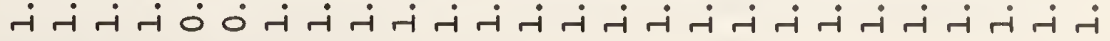

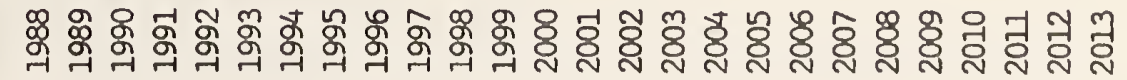




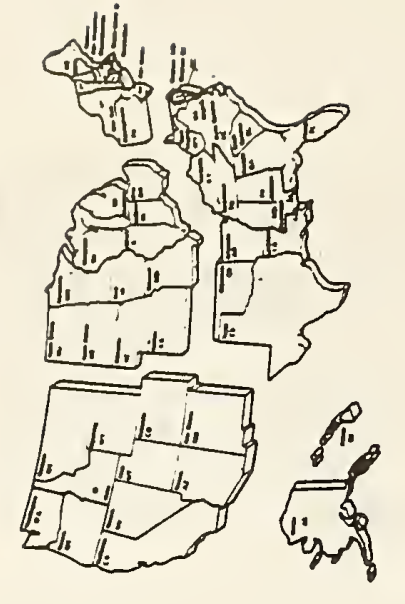

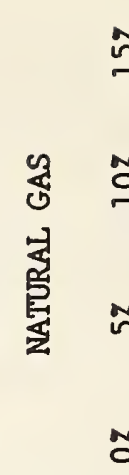

\begin{tabular}{l|} 
ñ \\
oै \\
ñ \\
ñ
\end{tabular}

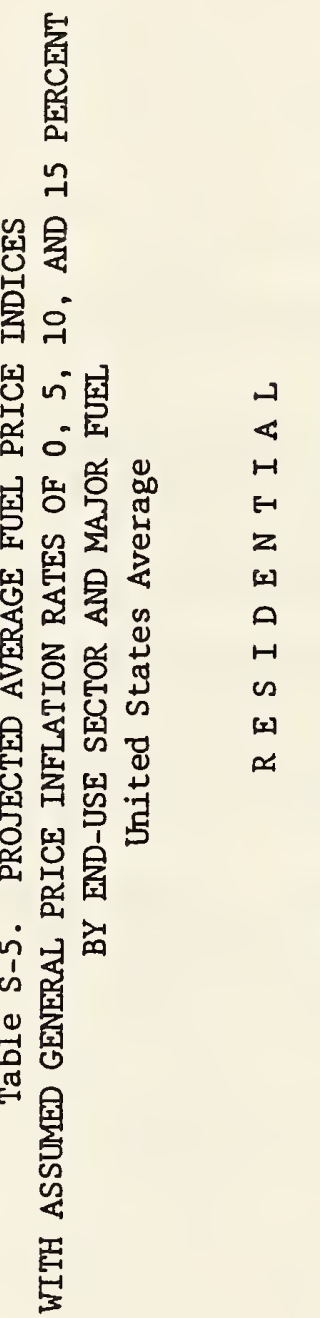

密 号

ติ่

$\stackrel{a}{a}$

选

in

re

กี่

oㅡ

ó

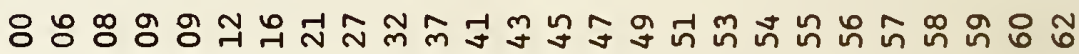
-i નં

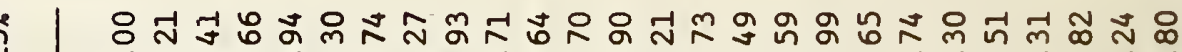

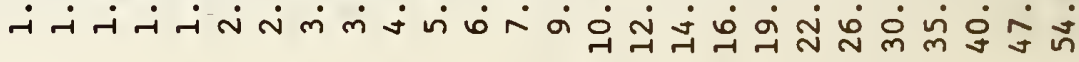

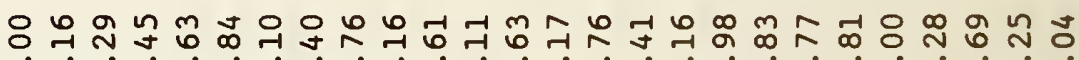

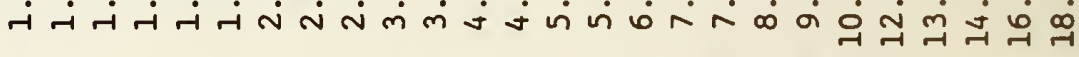

8 ન

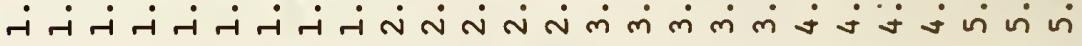

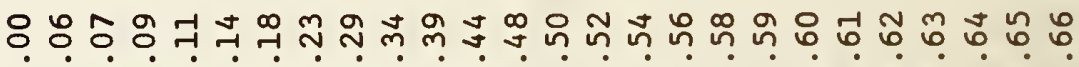

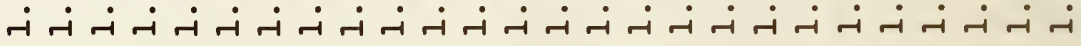
음

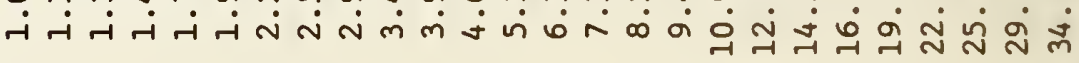

8웅ㄱำ

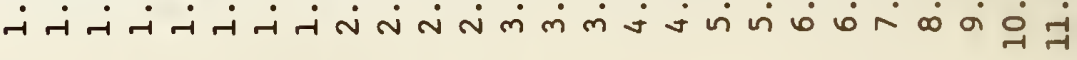

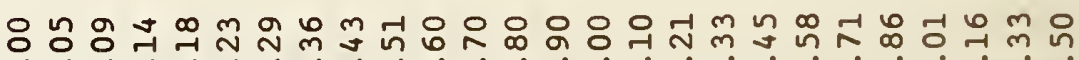

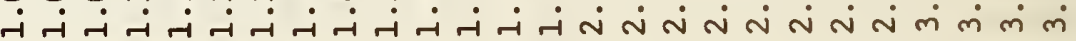

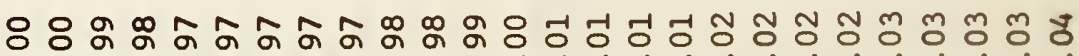

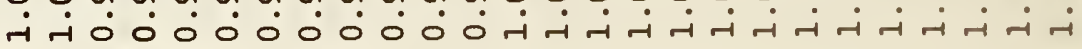

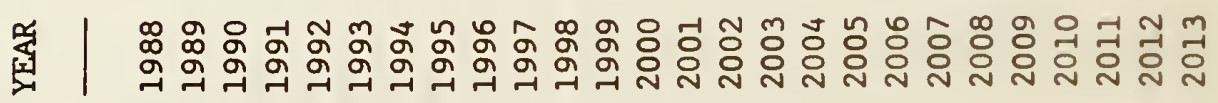


SHEET (See instructions)

1. PUBLICATION OR
REPORT NO.
NISTIR-85/3273-

2. Performing Organ. Report No. 3. Publication Date

NISTIR-85/3273-3

November 1988

4. TITLE AND SUBTITLE

Energy Prices and Discount Factors for Life-Cycle Cost Analysis 1988: Annual

Supplement to NBS Handbook 135 and NBS Special Publication 709.

5. AUTHOR(S)

Barbara C. Lippiatt and Rosalie T. Ruegg

6. PERFORMING ORGANIZATION (If joint or other than NBS, see instructions)

7. Contract/Grant No.

\section{NATIONAL BUREAU OF STANDARDS \\ U.S. DEPARTMENT OF COMMERCE \\ GAITHERSBURG, MD 20899}

8. Type of Report \& Period Covered

Annual

9. SFONSOFING CFGANIZATION WAAME AND COMPLETE ADCNESS (StrERE, CITY, State, ZIF)

U.S. Department of Energy

office of the Assistant Secretary

Federal Programs office

for Conservation and Renewable Energy

10. SUPPLEMENTARY NOTES This report is comprised of energy price and discount factor tables which are annual updates of tables referenced in NBS Handbook 135 and NBS Special Publication 709. Only minor changes have been made to this previously WERB-approved material.

Document describes a computer program; SF-185, FIPS Software Summary, is attached.

11. ABSTRACT (A 200-word or less foctual summary of most significant information. If document includes a significant bibliography or literature survey. mention it here)

This is the 1988 annual edition of energy prices and discount factors for performing life-cycle cost analyses of energy conservation and renewable energy projects. It supports the Federal life-cycle costing methodology as described in NBS Handbook 135 (HB 135) and private sector life-cycle cost analysis as described in NBS Special Publication 709 (SP 709). Tables A, B, and C are revisions of appendices A, B, and $\mathrm{C}$, respectively, of $\mathrm{HB}$ 135. Tables $\mathrm{A}(7 \%), \mathrm{Ba}$, and $\mathrm{C}$ apply to Federal energy conservation and renewable energy projects. Tables A (10\%), Bb, and C apply to Federal projects that require energy price forecasts but are not primarily energy conserving. Tables $\mathrm{S}$, in the last section of this report, are revisions to appendix B, Part I of SP 709 and are provided for the convenience of private sector analysts wishing to make use of Federal energy price forecasts.

12. KEY WORDS (Six to twelve entries: alphabetical order: capitalize only proper names; and separate key words ty semicolons average fuel prices; energy conservation; energy price forecasts; Federal Energy Management Program; life-cycle cost analysis; single present worth discount factors; uniform present worth discount factors

\section{AVAILABILITY}

X Unlimited

For Official Distribution. Do Not Release to NTIS

Order From Superintendent of Documents, U.S. Government Printing Office, Washington, DC 20402.

14. NO. OF PRINTED PAGES

60

15. Price

X Order From National Technical Information Service (NTIS), Springfield, VA 22161

$\$ 13.95$ 



